\title{
On the Logic of Belief and Propositional Quantification
}

\author{
Yifeng Ding $^{1}$ ( )
}

Received: 10 February 2020 / Accepted: 11 February 2021 / Published online: 5 April 2021

(C) The Author(s) 2021

\begin{abstract}
We consider extending the modal logic $\mathrm{KD} 45$, commonly taken as the baseline system for belief, with propositional quantifiers that can be used to formalize natural language sentences such as "everything I believe is true" or "there is something that I neither believe nor disbelieve." Our main results are axiomatizations of the logics with propositional quantifiers of natural classes of complete Boolean algebras with an operator (BAOs) validating $\mathrm{KD} 45$. Among them is the class of complete, atomic, and completely multiplicative BAOs validating $\mathrm{KD} 45$. Hence, by duality, we also cover the usual method of adding propositional quantifiers to normal modal logics by considering their classes of Kripke frames. In addition, we obtain decidability for all the concrete logics we discuss.
\end{abstract}

Keywords Modal logic · Doxastic logic Propositional quantifiers · Algebraic semantics · Pesudo-monadic algebras · Kripke incompleteness

\section{Introduction}

In this paper, we consider extending the modal logic $\mathrm{KD} 45$, commonly taken as the baseline system for belief, with propositional quantifiers that can be used to formalize natural language sentences such as "everything I believe is true" or "there is something that I neither believe nor disbelieve." Our main results are axiomatizations of the logics with propositional quantifiers of natural classes of complete Boolean algebras with an operator (BAOs) validating $\mathrm{KD} 45$. Among them is the class of complete, atomic, and completely multiplicative BAOs validating $\mathrm{KD} 45$. Hence, by duality, we

I thank Wesley Holliday for his numerous helpful suggestions and Fengkui Ju at the 2019 Modal Logic Workshop at Peking University for reminding me that $4^{\forall}$ strengthens 4 .

Yifeng Ding

yf.ding@berkeley.edu

1 Group in Logic and the Methodology of Science, UC Berkeley, Berkeley, CA, USA 
also cover the usual method of adding propositional quantifiers to normal modal logics by considering their classes of Kripke frames. In addition, we obtain decidability for all the concrete logics we discuss.

The present work can be seen as sitting at the intersection of two strands of literature: the doxastic logic literature, since we are extending $\mathrm{KD} 45$, and the literature on modal logics with propositional quantifiers, since we are extending with propositional quantifiers. In both bodies of literature, algebraic approaches are not particularly popular. Moreover, $\mathrm{KD} 45$ was not discussed in the literature of modal logics with propositional quantifiers until very recently [1]. To explain our motivation and potential contribution to the two bodies of literature in more detail, we use two subsections below.

\subsection{Dubious Principles and Possible-World Semantics}

Since Hintikka [2], modal logic has been indispensable for the study of intensional propositional operators like knowledge and belief. For the belief case, the system KD45 arose naturally as a baseline system. The reason may be that KD45 puts together the properties that we immediately recognize as what an ideal agent's belief (or an agent's ideal belief) should have: logical omniscience, consistency, and full introspection. Indeed, the modal rule and axioms in the standard axiomatization of $\mathrm{KD} 45$ can be matched precisely to these properties: the necessitation rule and $\mathrm{K}$ to logical omniscience, $\mathrm{D}$ to consistency, and 4 and 5 to introspection. The attitudes toward these idealizations vary (see, for example, more friendly views in [3] and Section 1.3 of [4] and much less friendly views in [5]), but the system KD45 remains central (for its most recent appearance, see [6] but also [7]).

Coming along with the syntactical formalism of modal logic is the possibleworld semantics based on possible-worlds and accessibility relations (namely Kripke frames). The use of possible-world semantics is perhaps mainly fueled by the correspondence and completeness results for most philosophically interesting modal formulas. When deciding which axioms to use, if we accept that possible-world semantics in general is appropriate, we may first find out the axioms' corresponding frame conditions. To quote David Lewis in [8, p. 19], "instead of asking the baffling question whether whatever is actual is necessarily possible, we could try asking: is the relation $R$ symmetric?" When we already have a strong intuition on which logic is the most appropriate (for whatever purpose), we may still want to use possible-world models to succinctly represent a consistent set of formulas describing a situation and then guide our syntactic reasoning in that situation. Completeness guarantees that this is always possible.

For the belief case, if we are not venturing below $\mathrm{K}$, the standard possible-world semantics based on Kripke frames is always appropriate by Sahlqvist's completeness theorem $[9, \S 5.6]$, since the relevant axioms are D, 4, and 5, which are all Sahlqvist formulas. Moreover, all modal logics extending $\mathrm{KD} 45$ are Kripke-complete in the sense that they are complete with respect to the classes of Kripke frames on which they are valid [10]. Even with the addition of dynamic operators as in [11], semantics based on possible-worlds is still largely appropriate, and many such extensions start 
with possible-world semantics. While it is well known that there are Kripke incomplete logics [12], meaning that no classes of Kripke frames can validate precisely the theorems in those logics, perhaps, when studying belief operators, Kripke frames are always enough for us, and there is nothing that can "banish" us from, to borrow from David Lewis again, "a doxastic logician's paradise"?

As another way of extending the language of Doxastic logic, consider propositional quantifiers. While we naturally quantify over propositions in both ordinary and philosophical discourses about belief, the addition of propositional quantifiers is not given much attention in the literature. Can we repeat the success story of the Kripke semantics here again, or are we in the situation that, with propositional quantifiers, we gain enough expressivity so that Kripke frames with their well-documented quirks in the literature on Kripke incompleteness lead to unwanted validities? Note that if there are formulas in the extended language such that, on the one hand, they are valid on Kripke frames validating a logic $L$, and on the other hand, we have strong reasons to at least treat them as optional and study and use extensions of $L$ without them, Kripke frames must go.

Indeed, a number of new principles about belief that seem conceptually significant are formalizable in the extended language.

- "One believes that everything one believes is true" is formalized as $\mathrm{B} \forall p(\mathrm{~B} p \rightarrow$ $p)$.

- "If no matter what $p$ stands for, one believes that $\varphi$, then one believes that no matter what $p$ stands for, $\varphi$ " is formalized as $\forall p \mathrm{~B} \varphi \rightarrow \mathrm{B} \forall p \varphi$.

- "There is a proposition that the agent takes to be consistent and to settle everything" can be formalized as $\exists q(\widehat{\mathrm{B}} q \wedge \forall p(\mathrm{~B}(q \rightarrow p) \vee \mathrm{B}(q \rightarrow \neg p)))$.

Conceptually, then, we can ask: if we would like to take all the idealizations encoded in $\mathrm{KD} 45$ on board, should we also adopt or are we already committed to some of the principles above, once we add propositional quantifiers into our language?

Let us focus on the first principle, which we call Immod: "one believes that everything one believes is true." Even for idealized agents or idealized beliefs, as axiomatized by $\mathrm{KD} 45$, it seems that Immod should not be included in a logic of belief. After all, the idealizations we are granting here are only about logic and introspection and do not warrant the truth of the uncertain beliefs that we choose to believe. Immod should be distinguished from "for every proposition $\mathrm{p}$, one believes that if she believes that $\mathrm{p}$ then $\mathrm{p}$ " (with the "if ... then ..." here being the material implication). This principle, when formalized as $\forall p(\mathrm{~B}(\mathrm{~B} p \rightarrow p))$, is merely the universalization of a simple consequence of the negative introspection axiom. The crucial difference between this principle and Immod is that Immod says that one believes the totality of one's belief to be true, while $\forall p \mathrm{~B}(\mathrm{~B} p \rightarrow p)$ says only that for every proposition $p$, when considered individually, one believes that if $p$ is believed, then $p$ is true.

More concretely, we can take an agent who has credences about a real number $x$ randomly generated (perhaps by an unending sequence of fair coin flips) from the interval $[0,1]$. For all measurable $X \subseteq[0,1]$, the agent's credence that $x \in X$ is just the measure of $X$. In addition, in this simple example, it seems not against our intuitive understanding of the concept of outright belief that the agent can simply 
believe precisely those propositions with credence 1 . $^{1}$ Then, for all $a \in[0,1]$, the agent believes that $x \in[0,1] \backslash\{a\}$ since $[0,1] \backslash\{a\}$ is measure 1 . However, the agent does not believe that for all $a \in[0,1], x \in[0,1] \backslash\{a\}$ since $\bigcap_{a \in[0,1]}([0,1] \backslash\{a\})=\varnothing$, which is not measure 1 . Hence the agent in this situation does not believe that all her beliefs are true.

The above of course does not constitute a decisive argument that Immod is not valid for ideal agents or ideal beliefs axiomatized by $\mathrm{KD} 45$. But we hope that at least we have demonstrated some interest that people might have in considering a logic without Immod. On the semantic side, though, as we will show in Section 2, if we adopt the standard possible-world semantics, Immod as formalized by $\mathrm{B} \forall p(\mathrm{~B} p \rightarrow$ $p$ ) is valid on any Kripke frame that validates $\mathrm{KD} 45$. Indeed, it is valid so long as the accessibility relation is shift-reflexive, ${ }^{2}$ regardless of which domain of propositions (as represented by subsets of possible-worlds) we choose for the propositional quantifiers to range over and regardless of whether the domain varies from world to world. In other words, if we constrain ourselves with the standard possible-world semantics, the space of logics between $\mathrm{KD} 45$ and $\mathrm{KD} 45$ plus Immod is closed to us.

To allow for modesty above $\mathrm{KD} 45$, we will turn to algebraic semantics. In algebraic semantics, propositions, instead of possible worlds, are first-class citizens that naturally form Boolean algebras when ordered by logical strength. Then, propositional quantifiers are interpreted in these algebras of propositions by the meet operation since, intuitively, for example, "everything I believe is true" is the conjunction of all instances of "if I believe that $p$ then $p$." Specifically, we will use what were used in the first algebraic semantics for a KD4 5 belief operator in [14]: proper filter algebras, except that we will consider only those whose underlying Boolean algebra is complete ${ }^{3}$ in the sense that arbitrary, not just finite, meets and joins exist. We believe there can be an independent metaphysical argument for why the Boolean algebra of propositions should at least be complete, but such an argument falls outside the scope of this paper. For our purposes, the completeness condition is merely a condition with which we can show, in a way that does not use any special property of the belief operator $\mathrm{B}$, that all formulas, including those like $\forall p \varphi$, have well-defined semantic values. In other words, lattice completeness is a language-and-logic-blind condition guaranteeing that our algebraic semantics works.

While proper filter algebras allow modesty, they are not completely conceptually innocent beyond KD45 though. A strengthened introspection axiom, which we call $4^{\forall}$, is valid on these algebras. This new axiom $4^{\forall}$ intuitively reads: if the agent believes every instance of $\varphi$, then the agent believes that she believes every instance of $\varphi$. In the formal language to be introduced in full later, $4^{\forall}$ is $\forall p \mathrm{~B} \varphi \rightarrow \mathrm{B} \forall p \mathrm{~B} \varphi$. However, unlike Immod, we find $4^{\forall}$ well-motivated, especially when we are considering extending KD45. Typically, and especially under idealization, we take our judgment about our internal state, like believing $\varphi$ or not, as infallible. If so, it is not

\footnotetext{
${ }^{1}$ Note that this does not rely on the agent's belief being reduced to credence in any way. However, see [13].

${ }^{2}$ A binary relation $R$ is shift-reflexive if and only if for all $x$ and $y, x R y$ implies $y R y$. Shift-reflexivity follows from Euclidicity, the first-order correspondence of the axiom 5.

${ }^{3}$ Since the word "complete" is also used for saying that a logic is complete, we sometimes use "lattice complete" to express this idea.
} 
just that we are in a position to believe that we believe $\varphi$ when we do believe $\varphi$. The aggregation of arbitrarily many such infallible judgments is still infallible (contrary to a large aggregation of credence 1 yet fallible propositions) and to be believed by us (or idealized versions of us). The formula $4^{\forall}$ precisely formalizes this reasoning step.

Corresponding to this idea is the fact that a proper filter algebra works by keeping a proper filter of propositions in the underlying Boolean algebra as the filter of "believed propositions" and interprets $\mathrm{B} \varphi$ to either the top element or the bottom element depending on whether $\varphi$ is interpreted as a "believed proposition" or not. If the proposition expressed by $\varphi$ is "believed", then $\mathrm{B} \varphi$ is interpreted as the top element and otherwise the bottom element. More technically, proper filter algebras can be understood as Boolean algebras with an operator that validate $\mathrm{KD} 45$ and also has the special property that the operator sends propositions to either the top element or the bottom element. Intuitively, then, from the agent's perspective, a formula $\mathrm{B} \varphi$ is as true as tautologies are once true and is as false as contradictions are once false. ${ }^{4}$ Hence, it is not hard to check that $4^{\forall}$ is valid, since we are essentially only considering the two-element Boolean algebra once we treat $\mathrm{B} \varphi$ as a whole.

But will this class of complete proper filter algebras validate any other formulas whose interpretation might be unwelcome? Our axiomatization suggests that the answer is no. We will show that the logic of complete proper filter algebras is axiomatized by $\mathrm{KD} 4{ }^{\forall} 5 \Pi$, obtained by adding to $\mathrm{KD} 45$ the usual $\Pi$-principles, namely those axioms about propositional quantifiers that are analogous to the axioms about first-order quantifiers, and then strengthening 4 to $4^{\forall}$. Since the $\Pi$-principles encode only the quantificational axioms, like instantiation and universalization, the only conceptual leap in this axiomatization is from 4 to $4^{\forall}$.

\subsection{Axiomatizability for Modal Logics with Propositional Quantifiers}

Now we turn to a more technical side and connect our work to the literature on modal logics with propositional quantifiers. The systematic technical study of propositional quantifiers is arguably initiated in Fine's dissertation [15], though already in Kripke's [16], propositional quantifiers are discussed. Also around the same time as Fine's dissertation were Bull's [17] and Gabbay's [18]. Soon after his dissertation, Fine summarized and extended his results in [19]. From these early papers, we can already see a wide range of semantic choices, especially about the domain of

\footnotetext{
${ }^{4}$ Of course a tautology like $p \rightarrow p$ and a formula like $\mathrm{B} p$ have different truth conditions, regardless of whether $\mathrm{B} p$ is true or not. So the proper filter algebras we consider are not representing propositions obtained by way of metaphysical (or a priori) equivalence where two sentences $\varphi$ and $\psi$ express the same proposition iff necessarily (or a priori) $\varphi$ and $\psi$ are either both true or both false. The proper filter algebras represent algebras of propositions obtained for a particular agent in a particular situation by stipulating that two sentences $\varphi$ and $\psi$ express the same proposition iff the agent is certain in that situation that either $\varphi$ and $\psi$ are both true or both false. For those who are unsatisfied with the restrictedness of proper filter algebras, we will show that we can, without changing the logic, consider all complete Boolean algebras with an operator validating $\mathrm{KD} 45$. In this way, we can be more neutral on what count as "propositions". However, it is non-trivial to see that $4^{\forall}$ is valid on all such algebras, and we devote the whole of Section 3 to this issue.
} 
propositions that $\forall p$ can quantify over (which is naturally encoded in general frames). Bull and Gabbay in the above-cited papers also identified two ways to refute Barcan's schema $\forall p \square \varphi \rightarrow \square \forall p \varphi$ through varying the domain of propositions for quantifiers across possible-worlds and through generalizing accessibility relations to neighborhood functions. In a completely non-technical paper [20], we also saw perhaps the earliest proposal of treating $\forall p$ as quantifying directly over objects in a lattice of propositions, a proposal perhaps inspired by the philosophical stance defended in that paper. Since then, there has been a steady stream of interest devoted to this topic, with general theoretical results focusing on expressive power under the standard possible-world semantics ([21-24]), specific results mostly establishing non-axiomatizability ([25-33]) with the exception of [31] and [33], and more application-oriented works: [1, 34-39].

A remarkable phenomenon when studying unimodal logic with propositional quantifiers on Kripke frames, where every set of possible-worlds counts as a proposition that $\forall p$ can quantify over, is the seeming existence of what we call an "axiomatizability boundary": there seems to be a line in the order structure of classes of Kripke frames of usual normal modal logics such that, below this line, the logics of those classes of frames with propositional quantifiers are extremely complex (often recursively equivalent to full second-order logic) and non-axiomatizable, while above this line, the logics with propositional quantifiers are suddenly decidable. Of course, we need to define what is "usual" for this "axiomatizability boundary" concept to make sense. A very preliminary step is to consider first the lattice of Kripke frame classes corresponding to the logics in the modal logic cube (Fig. 1). We see that Fine's 1970 paper [19] sets the boundary between S4 and S5 and between B and S5. Kaminski's result [21] pushes the boundary further from S4 to S4.2. However, where the boundary lies in the direction from S5 to KD45 and KB5 remained open. In this paper, we will show that the boundary can be pushed from the decidable side to KD45: the logic with propositional quantifiers of Kripke frames validating KD45 is decidable.

Fig. 1 The frame classes cube. Darker shade means the corresponding logic with propositional quantifiers is non-axiomatizable. No shade means decidability established, and light shade means decidability unknown

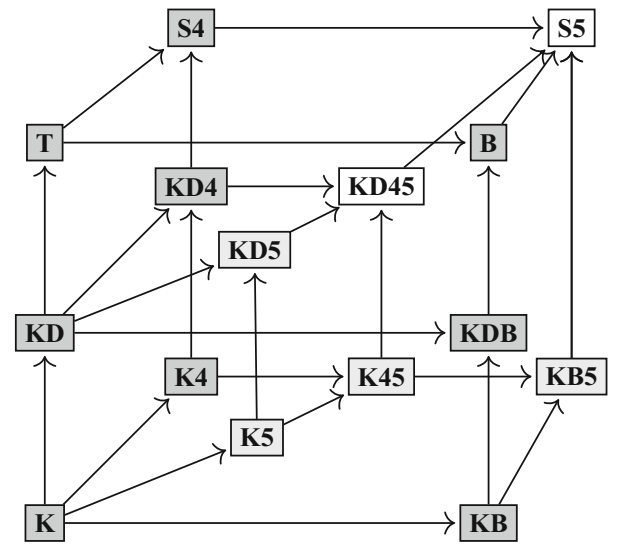


We may just focus on Kripke frames if we are only aiming at pushing the axiomatizability boundary. But also hard to ignore in this literature is a severe lack of an algebraic approach (until very recently; [40, 41], and [42]). In particular, when propositional quantifiers are added to a modal logic $L$ in the basic language, this is usually done by considering some class of Kripke frames on which $L$ is valid and then generating the logic with propositional quantifiers of this class of Kirpke frames. The main variability is in changing the domain of propositions for the propositional quantifiers to quantify over, and this is often achieved by considering general frames whose underlying Kripke frames are frames of $L$. Then in a general frame, the domain for interpreting propositional variables and for propositional quantifiers is naturally the set of admissible propositions. A problem with this approach, however, is that when we take $\forall p$ to mean "no matter what $\mathrm{p}$ stands for," which is the interpretation we are interested in here, the semantics must validate the full instantiation axiom $\forall p \varphi \rightarrow \varphi[\psi / p]$ where $\varphi[\psi / p]$ is the result of substituting $p$ with $\psi$ (with necessary renaming of bound propositional variables). In general frames, validating the full instantiation axiom often involves putting a so-called "closed under formula" condition, which seems to be dependent on the choice of $L$.

In the algebraic semantics for propositional quantifiers, the lattice completeness of an algebra of propositions ensures the well definedness of the semantic value of all formulas and the validity of the full instantiation axioms. While the lattice completeness condition is usually not necessary for this purpose, it is blind to the choice of language and logic. The semantics also directly models the intended interpretation of $\forall p$ : the semantic value of $\forall p \varphi$ on an algebra is the meet of all the possible semantic values of $\varphi$ as we reevaluate $p$ to all elements in the algebra. Hence, the algebraic method, in contrast to the above possible-world-based method, of adding propositional quantifiers to $L$ is to take the logic, in the language extended with propositional quantifiers, of the complete Boolean algebras with operators validating L. One can then investigate the result of imposing atomicity and/or complete multiplicativity. In particular, if both conditions are imposed, we recover the version of possible-world-based method of extension where all subsets count as propositions.

The algebraic approach poses also a series of natural open questions, and we will list some in the concluding section of this paper. An example, relating to the above phenomenon of the "axiomatizability boundary", is this: how would a shift from Kripke frames to complete BAOs affect the boundary? Will the boundary move or even blur in the sense that we will see logics undecidable yet not as complex as theories like the second-order theory of arithmetic? In all the proofs of nonaxiomatizability, atomicity is at least implicit in the set-up, if not directly used. It is not our ambition in this paper to settle questions at this level of generality though. Our aim is merely to initiate this program by focusing on a very special case: the case of extending KD45 with propositional quantifiers in an algebraic way. And we obtain the following results from a few more general theorems that we will establish along the way:

- If we consider all complete BAOs validating $\mathrm{KD} 45$, the resulting logic is $\mathrm{KD} 4{ }^{\forall} 5$ П. Note that in principle we can consider the wider class of BAOs which happen to make the semantics well-defined and also validate $\mathrm{KD} 45$. In particular, 
the Lindenbaum algebra of $\mathrm{KD} 45 \Pi$ is such an algebra. So if we drop the lattice completeness condition, we get $\mathrm{KD} 45 \Pi$. Conditioning on $\mathrm{KD} 45 \Pi \subsetneq \mathrm{KD} 4{ }^{\forall} 5 \Pi$, this means that lattice completeness is not inert: it strengthens 4 into $4^{\forall}$.

- Imposing complete multiplicativity of B amounts to adding Immod (or Barcan's schema) to $\mathrm{KD} 4{ }^{\forall} 5 \Pi$.

- Imposing atomicity amounts to adding a much more complicated formula, which we will call At, to $\mathrm{KD} 4{ }^{\forall} 5 \Pi$.

- Hence, if both conditions are imposed, the resulting logic is $\mathrm{KD} 4^{\forall} 5$ ПImmodAt. By duality theory, then, this is the logic of serial, transitive, and Euclidean Kripke frames.

- Finally, all the logics above are decidable. Hence the "axiomatizability boundary" is pushed to KD45 and does not change when we switch from Kripke frame to complete BAOs.

\subsection{Organization}

The rest of the paper is organized as follows. In Section 2, we formally define the language and algebraic semantics, and then introduce the necessary axioms and systems with some of their logical relations; we then show how the algebraic semantics can invalidate Immod while to a certain extent frame-based semantics cannot. In Section 3 , we show that $4^{\forall}$, and hence the logic $\mathrm{KD} 4^{\forall} 5 \Pi$, is valid on all complete $\mathrm{KD} 45$ algebras. In Section 4 , we show that $\mathrm{KD} 4{ }^{\forall} 5 \Pi$ is complete with respect to the class of all complete proper filter algebras. Since complete proper filter algebras are also complete $\mathrm{KD} 45$ algebras, $\mathrm{KD} 4{ }^{\forall} 5 \Pi$ axiomatizes the logic of both complete KD4 5 algebras and complete proper filter algebras and also any class of algebras in between. This is the longest section of the paper, in which we need to prove two technical lemmas. The first lemma is an analog of the quantifier elimination used to show the completeness of S5 $\Pi$ by Fine. While we do not need a full quantifier elimination, we need to show that the quantifiers can be separated from unmodalized propositional variables and pulled out from the scopes of modal operators so that we can translate formulas into a first-order language about Boolean algebras with two named elements. The second lemma at its core says that the first-order logic of the quotients of complete Boolean algebras is just the first-order logic of Boolean algebras. While this seems to be a natural proposition of independent interest, to the best of our knowledge, it has not been shown previously. In Section 5, we extract more results from the proofs in Section 4 and establish two general completeness theorems. From them, the logics resulting from imposing atomicity and complete multiplicativity to algebras naturally follow. We then show a general decidability theorem, from which the decidability of all the particular logics discussed follows. In the last section, Section 6, we conclude with directions of future research.

\section{Syntax, Semantics, Logics, and the Problem of Immod}

The propositional language with a belief operator and propositional quantifiers is defined as follows. 
Definition 2.1 Define the language $\mathscr{L} \Pi$ by the following grammar:

$$
\varphi::=p|\top| \neg \varphi|(\varphi \wedge \varphi)| \mathrm{B} \varphi \mid \forall p \varphi
$$

where $p \in$ Prop, a set of propositional variables. ${ }^{5}$ We adopt the usual abbreviations, and in particular we frequently write $\perp$ for $\neg \top, \widehat{\mathrm{B}}$ for $\neg \mathrm{B} \neg$, and $\exists p$ for $\neg \forall p \neg$. The free and bound occurrences of propositional variables are defined as in first-order logic. As is common in first-order logic, we write $\varphi(p)$ to note that $\varphi(\psi)$ is then the result of replacing the free occurrences of $p$ in $\varphi$ by $\psi$ with necessary renaming of bound variables.

Now we turn to semantics. Algebraic semantics starts with a Boolean algebra of propositions, and every formula will be evaluated to one of the propositions in it. If we define Boolean algebras simply by the laws of conjunction and negation, then the semantics seems to lack motivation independent of the logic we want it to generate. However, it is also well known (see Chap. 4 of [43]) that they can be equivalently defined as partial orders with greatest lower bounds (meets), least upper bounds (joins), and complements, or more specifically, complemented distributive lattices. Thus, a Boolean algebra can be seen as representing propositions that form a complemented distributive lattice once ordered by their strength. Then $\top, \wedge, \vee$, and $\neg$ are interpreted uncontroversially as the top element, the meet (greatest lower bound) operation, the join (least upper bound) operation, and the complementation operation, respectively.

In the same fashion, $\forall p \varphi$ should express the proposition that is the meet of all propositions expressible as $\varphi$ while the proposition expressed by $p$ ranges over all propositions in the algebra. Since there are possibly infinitely many such propositions expressible by $\varphi$, we make a further assumption about the Boolean algebra of propositions we study in this paper: they must be complete in the sense that every set of elements has a meet. As for the belief operator, the most general representation we can have is to use an arbitrary function on each algebra of propositions. But since our concern in this paper is to study the logics of belief at least as strong as $\mathrm{KD} 45$, we need to make corresponding assumptions on this function representing the belief operator. The following definition summarizes the assumptions we make.

Definition 2.2 A KD45 algebra is a pair $\mathscr{B}=\langle B, \square\rangle$ where

- $\quad B$ is a non-trivial Boolean algebra with $\top$ being its top element, $\neg$ its complementation operation, and $\wedge$ its meet relation, and

- $\square$ is a unary function on $B$ such that for all $a, b \in B$,

$\square \top=\top, \square(a \wedge b)=\square a \wedge \square b, \neg \square \neg \top=\top, \square a=\square \square a$, and $\neg \square a=\square \neg \square a$.

When we need to distinguish the operations from different algebras, we will subscript the operations by the algebra they are from. For example, we may write $\wedge_{\mathscr{B}}$ for the meet operation of the Boolean algebra part of $\mathscr{B}$ or write $\wedge_{B}$ for the meet operation of $B$. We also write $\leq$, possibly with subscripts, for Boolean lattice orderings. We

\footnotetext{
${ }^{5}$ In contrast, $T$ can be viewed as a propositional constant.
} 
will frequently write $x \in \mathscr{B}$ instead of $x \in B$, which is already an abbreviation of $x$ being in the carrier set of $B$. The usual abbreviations for $\vee, \rightarrow, \leftrightarrow, \oplus, \backslash, \perp$, and $\diamond$ apply too.

A complete KD45 algebra is a KD45 algebra whose Boolean algebra part is a complete Boolean algebra. We use $\bigwedge$ and $\bigvee$ for arbitrary meets and joins in complete Boolean algebras. Again, subscripts are added and dropped as needed.

Then the language $\mathscr{L} \Pi$ can be interpreted on any complete KD45 algebra. To express semantic substitution, for any function $f: X \rightarrow Y$ and any $x \in X$ and $y \in Y$, we write $f[y / x]$ for the function that is identical to $f$ except that $f[y / x](x)=y$. This notation will be used by all the semantics we define in this paper.

Definition 2.3 For any complete KD45 algebra $\mathscr{B}$, a valuation $\theta$ on $\mathscr{B}$ is a function from Prop to $\mathscr{B}$. Then a valuation $\theta$ on $\mathscr{B}$ can be uniquely extended to $\tilde{\theta}: \mathscr{L} \Pi \rightarrow \mathscr{B}$ recursively by the following clauses:

$-\quad \tilde{\theta}(p)=\theta(p)$

- $\tilde{\theta}(\neg \varphi)=\neg \tilde{\theta}(\varphi), \tilde{\theta}(\varphi \wedge \psi)=\tilde{\theta}(\varphi) \wedge \tilde{\theta}(\psi)$, and $\tilde{\theta}(\top)=\top$;

- $\quad \tilde{\theta}(\mathrm{B} \varphi)=\square \tilde{\theta}(\varphi)$;

- $\tilde{\theta}(\forall p \varphi)=\bigwedge_{a \in \mathscr{B}} \widehat{\theta[a / p]}(\varphi)$.

A formula $\varphi \in \mathscr{L} \Pi$ is valid in a complete $\mathrm{KD} 45$ algebra $\mathscr{B}$ if for all valuations $\theta$ on $\mathscr{B}, \tilde{\theta}(\varphi)=\top$; otherwise we call it refutable in $\mathscr{B}$. A formula $\varphi$ is valid on a class of complete KD45 algebras if $\varphi$ is valid on each member of that class, and a set of formulas is valid on a class whenever every formula in the set is valid on the class. As usual, validity is denoted by $\vDash$.

One problem with the Definition 2.3 is that it is very general, and little structure of these complete KD45 algebras is revealed in the definition. While we will study them in detail in Section 3, we now introduce a very concrete semantics whose structures in which we evaluate formulas can be seen as directly modeling doxastic scenarios of ideal agents.

Definition 2.4 A proper filter algebra $\mathscr{B}$ is a pair $\langle B, F\rangle$ where $B$ is a Boolean algebra and $F$ is a proper filter of that Boolean algebra. A complete proper filter algebra is a proper filter algebra whose Boolean algebra part is a complete Boolean algebra. We will write $F_{\mathscr{B}}$ if the context is not clear enough.

Definition 2.5 For any complete proper filter algebra $\mathscr{B}=\langle B, F\rangle$, a valuation $\theta$ is a function from Prop to $\mathscr{B}$. Any valuation $\theta$ on $B$ extends to a $\mathscr{L} \Pi$-valuation $\tilde{\theta}: \mathscr{L} \Pi \rightarrow B$ given by:

- the same clauses for propositional variables $p \in$ Prop, connectives $\top, \neg, \wedge$, and $\forall p$ as in Definition 2.3, and

- $\tilde{\theta}(\mathrm{B} \varphi)=\top$ when $\tilde{\theta}(\varphi) \in F$ and otherwise $\tilde{\theta}(\mathrm{B} \varphi)=\perp$.

The concept of validity is defined as in Definition 2.3. 
As we have discussed, a proper filter algebra can be seen as representing the propositions individuated by equivalence up to subjective certainty in a concrete doxastic scenario, with the proper filter representing the believed propositions in the scenario. That the believed propositions should form a proper filter comes from the assumption that the agent is logically competent and never believes in blatantly false propositions. That $\tilde{\theta}(\mathrm{B} \varphi)$ is always either $\top$ or $\perp$ depending on whether $\tilde{\theta}(\varphi)$ is in the filter of believed propositions or not comes from the assumption that the agent is sufficiently introspective. Proper filter algebras were first seen in [14] as models for beliefs.

The connection between proper filter algebras and KD45 algebras is this: proper filter algebras naturally correspond to those KD45 algebras whose $\square$ operator's range is $\{\top, \perp\}$. In [44], KD45 algebras are called pseudo-monadic algebras, and those with the said property are called well-connected ones, so here we call the above property well-connectedness too.

The correspondence can be easily specified. For any proper filter algebra $\langle B, F\rangle$, we can define a $\square^{F}$ by $\square^{F} a=\top$ if $a \in F$ and $\perp$ otherwise. Then $\left\langle B, \square^{F}\right\rangle$ is the well-connected KD45 algebra corresponding to $\langle B, F\rangle$. Conversely, given a wellconnected KD45 algebra $\langle B, \square\rangle$, we can define $F^{\square}=\{a \in B \mid \square a=\top\}$. Then, $\left\langle B, F^{\square}\right\rangle$ is the corresponding proper filter algebra. It is easy to verify that these two constructions are both bijections and are inverse of each other. Moreover, the semantic value of every formula is preserved for any valuation $\theta$ when we replace either a $\square$ operator by the corresponding filter $F^{\square}$ or vice versa. We can also show that the correspondence is a natural isomorphism between the category of proper filter algebras and the category of well-connected KD45 algebras. But for our purposes in this paper, this step is unnecessary.

With the semantics of interest defined, we now move on to define logics. The interpretation of $\forall p \varphi$ above in the algebraic semantics is informed by its intended reading: "for all proposition $p, \varphi$." Given this reading, even without formal semantics, the following axiom schemas and rules for propositional quantifiers, which we call the П-principles, should be most certain:

- Dist $: \forall p(\varphi \rightarrow \psi) \rightarrow(\forall p \varphi \rightarrow \forall p \psi)$,

- Inst : $\forall p \varphi \rightarrow \varphi[\psi / p]$, where $\psi$ is subtitutable for $p$ in $\varphi$ and $\varphi[\psi / p]$ is the result of replacing all free occurrences of $p$ in $\varphi$ by $\psi$,

- Vacu : $\varphi \rightarrow \forall p \varphi$, if $p$ is not free in $\varphi$,

- Univ : whenever $\varphi$ is a theorem, $\forall p \varphi$ is also a theorem.

Just like a normal modal logic in full generality is defined as a set of formulas that contains all instances of propositional tautologies and the $\mathrm{K}$ axiom schema and is closed under the necessitation and modus ponens rules, we can similarly define normal П-logics.

Definition 2.6 A normal $\Pi$-logic in a language $\mathscr{L} \supseteq \mathscr{L} \Pi$ is a set of formulas in $\mathscr{L}$ that contains all instances of propositional tautologies, $\mathrm{K}$ for $\mathrm{B}$, and the $\Pi$-principles, and is closed under the necessitation rule $\mathrm{Nec}$ for $\mathrm{B}$, the universalization rule Univ for $\forall p$ for all $p \in$ Prop, and modus ponens. 
In this paper, we only consider normal $\Pi$-logics. When we put names of axiom schemas with $\mathrm{K}$ and $\Pi$ together, we always mean the smallest normal $\Pi$-logic containing all instances of those axiom schemas. The ambient language should be clear from the context. For example, in this section we can write $\mathrm{K} \Pi$ for the smallest normal $\Pi$-logic and write $\mathrm{KD} 45 \Pi$ for the smallest normal $\Pi$-logic in $\mathscr{L} \Pi$ containing all instances of $D, 4$, and 5 . In a later section where we prove the main completeness theorem, we will consider extended languages.

Now that the syntax, semantics, and $\Pi$-logics are all formally defined, recall the three principles about belief we have seen in Section 1:

Immod : $\mathrm{B} \forall p(\mathrm{~B} p \rightarrow p), \quad \mathrm{BC}: \forall p \mathrm{~B} \varphi \rightarrow \mathrm{B} \forall p \varphi, \quad 4^{\forall}: \forall p \mathrm{~B} \varphi \rightarrow \mathrm{B} \forall p \mathrm{~B} \varphi$.

Now we may have 8 normal $\Pi$-logics extending $\mathrm{KD} 45 \Pi$ by choosing which ones of the above three axiom schemas to add. But the following observation is immediate.

Proposition 2.1 $\mathrm{KD} 45 \Pi 4^{\forall}=\mathrm{KD} 4^{\forall} 5 \Pi$ and $\mathrm{KD} 45 \Pi \mathrm{BC}=\mathrm{KD} 4^{\forall} 5 \Pi \mathrm{Immod}$.

Proof For the first equality, it is enough to show that we can prove all instances of 4 in $\mathrm{KD}_{4}{ }^{\forall} 5 \Pi$. But for any $\varphi$, letting $p$ be a propositional variable not free in $\varphi$, we have the following derivation:

- $\mathrm{B} \varphi \rightarrow \forall p \mathrm{~B} \varphi$

[Vacu]

$-\quad \forall p \mathrm{~B} \varphi \rightarrow \mathrm{B} \forall p \mathrm{~B} \varphi$

$-\mathrm{B} \forall p \mathrm{~B} \varphi \rightarrow \mathrm{BB} \varphi$

[Inst, K, modus ponens]

$-\mathrm{B} \varphi \rightarrow \mathrm{BB} \varphi$

[modus ponens]

To show that $\mathrm{KD} 45 \Pi \mathrm{BC}=\mathrm{KD} 4{ }^{\forall} 5 \Pi \mathrm{Immod}$, it is enough to notice that $\mathrm{KD} 45 \Pi$ easily derives the following implications:

- $(\forall p \mathrm{BB} \varphi \rightarrow \mathrm{B} \forall p \mathrm{~B} \varphi) \rightarrow(\forall p \mathrm{~B} \varphi \rightarrow \mathrm{B} \forall p \mathrm{~B} \varphi)$,

$-(\forall p \mathrm{~B}(\mathrm{~B} p \rightarrow p) \rightarrow \mathrm{B} \forall p(\mathrm{~B} p \rightarrow p)) \rightarrow \mathrm{B} \forall p(\mathrm{~B} p \rightarrow p)$,

- $((\forall p \mathrm{~B} \varphi \rightarrow \mathrm{B} \forall p \mathrm{~B} \varphi) \wedge \mathrm{B} \forall p(\mathrm{~B} p \rightarrow p)) \rightarrow(\forall p \mathrm{~B} \varphi \rightarrow \mathrm{B} \forall p \varphi)$.

This proposition shows that there can be at most 4 different normal $\Pi$-logics extending $\mathrm{KD} 45 \Pi$ : $\mathrm{KD} 45 \Pi$ itself, $\mathrm{KD} 4{ }^{\forall} 5 \Pi, \mathrm{KD} 45 \Pi$ Immod, and $\mathrm{KD} 45 \Pi \mathrm{BC}$. If they are all different, then we will have the simple 4-element Boolean algebra as shown in Figure 2. But are they all different?

With the algebraic semantics above, we can easily show that $\mathrm{KD} 4{ }^{\forall} 5 \Pi \nvdash$ Immod, matching our intuition in the introduction that Immod is refutable even for ideally introspective agents. Then we see that $\mathrm{KD} 4{ }^{\forall} 5 \Pi$ is strictly below $\mathrm{KD} 45 \Pi \mathrm{BC}$, and

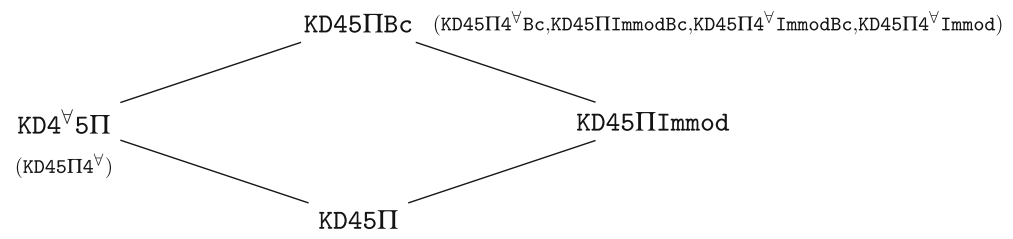

Fig. 2 Normal $\Pi$-logics extending $\mathrm{KD} 45 \Pi$ generated by $4^{\forall}$, Immod, and BC 
consequently $\mathrm{KD} 45 \Pi$ must also be strictly below $\mathrm{KD} 45 \Pi$ Immod by some simple Boolean reasoning. Thus with algebraic semantics, we can at least distinguish the lower left part from the upper right part. To show that $\mathrm{KD} 4{ }^{\forall} 5 \Pi \nvdash \mathrm{Immod}$, we only need a soundness theorem and countermodel. The soundness theorem is easy.

Theorem 2.1 For any $\varphi \in \mathrm{KD}^{\forall} 5 \Pi, \varphi$ is valid on all complete proper filter algebras.

Proof The only interesting axiom here is $4^{\forall}$. Pick an arbitrary complete proper filter algebra $\mathscr{B}$ and a valuation $\theta$ on it. Now for any $a \in \mathscr{B}, \widehat{\theta[a / p]}(\mathrm{B} \varphi)$ is either $\mathrm{\top}$ or $\perp$. If there is an $a \in \mathscr{B}$ such that $\widetilde{\theta[a / p]}(\mathrm{B} \varphi)=\perp$, then $\tilde{\theta}(\forall p \mathrm{~B} \varphi)=\perp$. Then trivially $\tilde{\theta}(\forall p \mathrm{~B} \varphi \rightarrow \mathrm{B} \forall p \mathrm{~B} \varphi)=\top$. On the other hand, if no such $a$ exists, then $\tilde{\theta}(\forall p \mathrm{~B} \varphi)=\top$, and hence $\tilde{\theta}(\mathrm{B} \forall p \mathrm{~B} \varphi)=\top$. Then trivially $\tilde{\theta}(\forall p \mathrm{~B} \varphi \rightarrow \mathrm{B} \forall p \mathrm{~B} \varphi)=$ $\top$. So $\tilde{\theta}(\forall p \mathrm{~B} \varphi \rightarrow \mathrm{B} \forall p \mathrm{~B} \varphi)=\top$ is valid either way.

To refute Immod in a countermodel, we first make the following useful and intuitive observation. It is intuitive because $\forall p(\mathrm{~B} p \rightarrow p)$ says that "everything the agent believes is true," and the filter $F_{\mathscr{B}}$ of a proper filter algebra represents the set of propositions the agent believes.

Proposition 2.2 For any complete proper filter algebra $\mathscr{B}$ and any valuation $\theta$ on it, $\tilde{\theta}(\forall p(\mathrm{~B} p \rightarrow p))=\bigwedge F_{\mathscr{B}}$.

Proof If $\tilde{\theta}(p) \in F_{B}$, then $\tilde{\theta}(\mathrm{B} p)=\top$, and hence $\tilde{\theta}(\mathrm{B} p \rightarrow p)=\tilde{\theta}(p)=\theta(p)$. If $\tilde{\theta}(p) \notin F_{B}$, then $\tilde{\theta}(\mathrm{B} p)=\perp$, and hence $\tilde{\theta}(\mathrm{B} p \rightarrow p)=\top$. Thus $\{\widetilde{\theta[a / p]}(\mathrm{B} p \rightarrow$ $p) \mid a \in \mathscr{B}\}$ is precisely $F_{\mathscr{B}}$ (note that $\top$ must be in $\left.F_{\mathscr{B}}\right)$. Then $\tilde{\theta}(\forall p(\mathrm{~B} p \rightarrow p)$ ) is the meet of this set, i.e., $\bigwedge F_{\mathscr{B}}$.

Given this observation, to refute $\mathrm{Immod}=\mathrm{B} \forall p(\mathrm{~B} p \rightarrow p)$, we only need to find a complete proper filter algebra $\mathscr{B}$ such that the meet of $F_{\mathscr{B}}$ is not in $F_{\mathscr{B}}$ : it is a non-principal filter.

Proposition 2.3 Immod is not valid on all complete proper filter algebras.

Proof Let $\mathscr{B}$ be a complete proper filter algebra where its Boolean algebra is $\wp(\mathbb{N})$, and its filter $F_{\mathscr{B}}$ is the set of all cofinite sets. Fix an arbitrary valuation $\theta$. Clearly $\bigwedge F_{\mathscr{B}}=\emptyset$, the bottom element. Using the previous proposition, $\tilde{\theta}(\forall p(\mathrm{~B} p \rightarrow p))=$ $\perp$, but $\perp \notin F_{\mathscr{B}}$. Thus $\tilde{\theta}$ (Immod) $=\perp$.

In fact, in this algebra, $\tilde{\theta}(\exists p(\mathrm{~B} p \wedge \neg p))=\top \in F_{B}$, so $\tilde{\theta}(\mathrm{B} \exists p(\mathrm{~B} p \wedge \neg p))=\top$. In other words, this agent believes that there is a proposition she falsely believes.

Now we show the difficulty of invalidating Immod using possible-world-based semantics. In full generality, allowing propositional contingency in the sense that the domain of propositions for each possible-world may vary, a frame is a tuple $F=\langle W, R, P\rangle$ where $W$ is a set (of possible worlds), $R$ is a binary relation (representing doxastic accessibility among worlds), and $P$ is a function from $W$ to 
$\wp(\wp(W))$ so that $P(w)$ represents the propositions that "exist" at $w$. To be fully general, we disregard any notion of "coherence" one might want to impose on $F$ (see [45] for some natural restrictions for $F$ ). A model is a tuple $\mathscr{M}=\langle F, w, V\rangle$ where $w$ is in the $W$ of $F$ and $V$ is a function from Prop to $V(w)$. To maintain that the range of $V$ is always in $P(w)$ as we evaluate formulas with modalities, we add it to the definition of a frame that $P$ is non-decreasing: if $w R w^{\prime}$ then $P(w) \subseteq P\left(w^{\prime}\right)$. Then the truth clauses can be defined as usual:

$$
\begin{array}{ll}
\langle F, w, V\rangle \vDash p & \Longleftrightarrow w \in V(p) ; \\
\langle F, w, V\rangle \vDash \neg \varphi \quad & \Longleftrightarrow\langle F, w, V\rangle \not \models \varphi ; \\
\langle F, w, V\rangle \vDash \varphi \wedge \psi & \Longleftrightarrow\langle F, w, V\rangle \vDash \varphi \text { and }\langle F, w, V\rangle \vDash \psi ; \\
\langle F, w, V\rangle \vDash \mathrm{B} \varphi \quad \Longleftrightarrow \text { for all } w^{\prime} \in R(w),\left\langle F, w^{\prime}, V\right\rangle \vDash \varphi ; \\
\langle F, w, V\rangle \vDash \forall p \varphi \quad \Longleftrightarrow \text { for all } X \in P(w),\langle F, w, V[X / p]\rangle \vDash \varphi .
\end{array}
$$

Here $R(w)=\left\{w^{\prime} \in W \mid w R w^{\prime}\right\}$. Then $\varphi$ being valid on a frame $F$ is defined as $\langle F, w, V\rangle$ making $\varphi$ true for all suitable $w$ and $V$.

A lot of standard questions can be asked about this semantics. But for now, observe that for any frame $F=\langle W, R, P\rangle$, if $R$ is shift-reflexive, meaning that every world in $R(w)$ is reflexive for all $w \in W$, then $F$ validates Immod. To see this, first note that for every $w$ in $W$ such that $w R w$ and any suitable valuation $V,\langle F, w, V\rangle \vDash \mathrm{B} p \rightarrow p$ simply by the truth clause of $\mathrm{B}$. Hence $\forall p(\mathrm{~B} p \rightarrow p)$ is also always true on reflexive points by the truth clause of $\forall p$. Then, it is clear again from the truth clause of $\mathrm{B}$ that for any $w \in W$ such that every $w^{\prime} \in R(w)$ is reflexive, $\langle F, w, V\rangle \vDash \mathrm{B} \forall p(\mathrm{~B} p \rightarrow p)$. Thus, if $R$ is shift-reflexive, Immod is validated. And in showing this, $P$ is totally unused.

Does the above argument show that possible-world semantics is totally unusable if we want to model scenarios where Immod is false? If one is looking for intuitive and "clean" models, then the argument does suggest that possible-world semantics is not useful. The success of possible-world semantics is partly due to the easy firstorder conditions corresponding to natural axioms. For the doxastic logic case, D, 4, and 5 correspond to seriality, transitivity, and Euclidicity, respectively. And from Euclidicity alone, shift-reflexivity follows. The above argument shows that if we want to model failure of Immod while validating $\mathrm{KD} 45$, we need to give up the appealing correspondence theory in the standard possible-world semantics. Nevertheless, it remains open what this semantics is capable of. We conjecture that with carefully chosen $P$ and $R$, we can refute Immod and even $4^{\forall}$ while validating KD45 П. However, we are less confident that the $R$ relation in that frame can be interpreted in a meaningful way.

\section{Soundness of $4^{\forall}$ on Complete KD45 Algebras}

In the last section, we have seen how complete proper filter algebras can be used to separate Immod from $\mathrm{KD} 4{ }^{\forall} 5 \Pi$ and hence separate $\mathrm{KD} 45 \Pi$ Immod and $\mathrm{KD} 45 \Pi \mathrm{BC}$ from $\mathrm{KD} 45 \Pi$ and $\mathrm{KD} 4{ }^{\forall} 5 \Pi$. We have also seen that complete proper filter algebras validate $4^{\forall}$ using the special property that the B operator always brings semantic values to either the top element or the bottom element. 
A natural question then is whether we can separate $4^{\forall}$ from $\mathrm{KD} 45 \Pi$ using algebraic semantics based on complete Boolean algebras at all. That is, whether we can refute $4^{\forall}$ if we do not assume the above special property about B. In this section, we show that we cannot. In fact, $4^{\forall}$, and hence $\mathrm{KD} 4^{\forall} 5 \Pi$, are valid on all complete KD45 algebras. For this, we need to extract more structure from complete KD45 algebras and view the algebraic semantics from a different perspective.

Definition 3.1 For any KD45 algebra $\mathscr{B}$, let $\mathrm{fp}(\mathscr{B})$ be the set $\{a \in \mathscr{B} \mid a=\square a\}$.

Lemma 3.1 For any KD45 algebra $\mathscr{B}, \mathrm{fp}(\mathscr{B})$ has the following properties.

- First, $f \mathrm{p}(\mathscr{B})=\{\square a \mid a \in \mathscr{B}\}=\{\diamond a \mid a \in \mathscr{B}\}$.

- Second, $\mathrm{fp}(\mathscr{B})$ is a subalgebra of $\mathscr{B}$. That is, $\mathrm{fp}(\mathscr{B})$ is closed under the complementation, the meet operation, and trivially the $\square$ operator of $\mathscr{B}$.

- Third, while $\left\langle\mathrm{fp}(\mathscr{B}), \top_{\mathscr{B}}, \neg \mathscr{B}, \wedge_{\mathscr{B}}\right\rangle$ is a complete Boolean algebra, it is not always a regular Boolean sublagebra of $\mathscr{B}$. In other words, when ordered as in $\mathscr{B}$ by $\leq \mathscr{B}, \mathrm{fp}(\mathscr{B})$ form a complete Boolean lattice whose complementation operation and finite meet operation are the same as in $\mathscr{B}$. However, it is not always the case that $\mathrm{fp}(\mathscr{B})$ is closed under arbitrary meets in $\mathscr{B}$.

Proof The first two points follow directly from the definition of KD45 algebras. For the third point, one can easily verify that the join of $X \subseteq \mathrm{fp}(\mathscr{B})$ within $\mathrm{fp}(\mathscr{B})$ is $\square \bigvee_{\mathscr{B}} X$. To see that for every $x \in X, x \leq \square \bigvee_{\mathscr{B}} X$, note that since $X \subseteq \mathrm{fp}(\mathscr{B}), x=$ $\square x$. Note also that $\square$ is monotone. Hence, given that $x \leq \bigvee_{\mathscr{B}} X, \square x \leq \square \bigvee_{\mathscr{B}} X$. Thus, $x \leq \square \bigvee_{\mathscr{B}} X$. Now suppose $y \in \mathrm{fp}(\mathscr{B})$ and for all $x \in X, y \geq x$. Then $y \geq \bigvee_{\mathscr{B}} X$. And then $y=\square y \geq \square \bigvee_{\mathscr{B}} X$. Hence $\square \bigvee_{\mathscr{B}} X$ is the least upper bound of $X$ in $\mathrm{fp}(\mathscr{B})$.

An example where the join of a subset of $\mathrm{fp}(\mathscr{B})$ in $\mathscr{B}$ is not in $\mathrm{fp}(\mathscr{B})$ is given below.

Definition 3.2 Let $\mathscr{N}$ be the set of non-principal ultrafilters in $\wp(\mathbb{N})$ and $W=$ $\mathbb{N} \sqcup \mathscr{N}$. For any $A \subseteq \mathbb{N}$, define $\mathscr{N}(A)=\{f \in \mathscr{N} \mid A \in f\}$. Then for any subset $X \subseteq W$, define $\square X$ by: $\square X=(X \cap \mathbb{N}) \cup \mathscr{N}(X \cap \mathbb{N})$. Finally, let $\mathscr{B}_{\mathbb{N}}=$ $\langle\langle\wp(W), W, W \backslash \cdot, \cap\rangle, \square\rangle$.

Proposition 3.1 $\mathscr{B}_{\mathbb{N}}$ is a complete KD45 algebra. Moreover, $\operatorname{fp}\left(\mathscr{B}_{\mathbb{N}}\right)$ is not closed under arbitrary join in $\mathscr{B}_{\mathbb{N}}$.

Proof Clearly $\mathscr{B}_{\mathbb{N}}$ is complete since the Boolean algebra base is a powerset algebra. Now we show that $\square$ satisfies all the relevant properties. Pick arbitrary $X, Y \in \mathscr{B}_{\mathbb{N}}$, and let $X_{0}=X \cap \mathbb{N}, X_{1}=X \cap \mathscr{N}, Y_{0}=Y \cap \mathbb{N}, Y_{1}=Y \cap \mathscr{N}$.

- $\square X \cap \square Y=\left(X_{0} \cup \mathscr{N}\left(X_{0}\right)\right) \cap\left(Y_{0} \cup \mathscr{N}\left(Y_{0}\right)\right)=\left(X_{0} \cap Y_{0}\right) \cup\left(X_{0} \cap \mathscr{N}\left(Y_{0}\right)\right) \cup\left(Y_{0} \cap\right.$ $\left.\mathscr{N}\left(X_{0}\right)\right) \cup\left(\mathscr{N}\left(X_{0}\right) \cap \mathscr{N}\left(Y_{0}\right)\right)=\left(X_{0} \cap Y_{0}\right) \cup \mathscr{N}\left(X_{0} \cap Y_{0}\right)=\square\left(X_{0} \cap Y_{0}\right)$. Here we used the fact that an ultrafilter contains $X_{0}$ and $Y_{0}$ iff it contains $X_{0} \cap Y_{0}$.

$-\square \varnothing=\varnothing \cup \mathscr{N}(\varnothing)=\varnothing$. 
$-\quad \square \square X=\square\left(X_{0} \cup \mathscr{N}\left(X_{0}\right)\right)=\left(\left(X_{0} \cup \mathscr{N}\left(X_{0}\right)\right) \cap \mathbb{N}\right) \cup \mathscr{N}\left(\left(X_{0} \cup \mathscr{N}\left(X_{0}\right)\right) \cap \mathbb{N}\right)=$ $X_{0} \cup \mathscr{N}\left(X_{0}\right)=\square X$.

$-\quad \neg \square X=\neg\left(X_{0} \cup \mathscr{N}\left(X_{0}\right)\right)=\left(\mathbb{N} \backslash X_{0}\right) \cup\left(\mathscr{N} \backslash \mathscr{N}\left(X_{0}\right)\right)=\left(\mathbb{N} \backslash X_{0}\right) \cup \mathscr{N}\left(\mathbb{N} \backslash X_{0}\right)=$ $(\neg X \cap \mathbb{N}) \cup \mathscr{N}(\neg X \cap \mathbb{N})=\square \neg X$. Hence by the previous part $\square \neg \square X=$ $\square \square \neg X=\square \neg X=\neg \square X$.

Thus, $\mathscr{B}_{\mathbb{N}}$ is a complete KD45 algebra. Note also that for every $n \in \mathbb{N},\{n\} \in \operatorname{fp}\left(\mathscr{B}_{\mathbb{N}}\right)$. However, $\mathbb{N}=\bigcup_{n \in \mathbb{N}}\{n\}$ is not in $\operatorname{fp}\left(\mathscr{B}_{\mathbb{N}}\right)$.

Semantically, every formula obtained by combining propositional variables with propositional operators (Boolean or modal) and propositional quantifiers defines a function from valuations in $\mathscr{B}^{\text {Prop }}$ to $\mathscr{B}$. Now we study these functions.

For simplicity and clarity, we fix an arbitrary complete KD45 algebra $\mathscr{B}$ and define $V=\mathscr{B}^{\text {Prop }}$. Greek letters " $\theta$ " and " $\gamma$ " are used to denote valuations in $V$ and " $f$ " and " $g$ " are used to denote functions from valuations in $V$ to $\mathscr{B}$. We also use the notations from lambda calculus to define functions.

The following definition then defines the meaning of the operators in terms of how they generate functions from $\mathscr{B}^{\text {Prop }}$ to $\mathscr{B}$.

Definition 3.3 First, for every $p \in$ Prop, define $[p]=\lambda \theta \in V, \theta(p)$. Then, for any $f, g: V \rightarrow \mathscr{B}$ and $p \in$ Prop, define:

$$
\begin{aligned}
f \wedge g & =\lambda \theta \in V, f(\theta) \wedge g(\theta) ; \\
\neg f & =\lambda \theta \in V, \neg f(\theta) ; \\
\square f & =\lambda \theta \in V, \square f(\theta) ; \\
\forall p f & =\lambda \theta \in V, \bigwedge\{f(\theta[a / p]) \mid a \in \mathscr{B}\} .
\end{aligned}
$$

Then $f \vee g, f \rightarrow g, f \leftrightarrow g, f \oplus g, \diamond f$, and $\exists p f$ are defined in the obvious way. We call the set of functions generated by $\neg, \wedge, \square$, and $\forall p$ for all $p \in$ Prop from $\{[p] \mid p \in \operatorname{Prop}\}$ the set of definable functions on $\mathscr{B}$.

The above definition also gives us an alternative way to define the semantics for $\mathscr{L} \Pi$.

Definition 3.4 Recall that we have defined $[p]=\lambda \theta \in V, \theta(p)$. We can then extend this notation to all formulas in $\mathscr{L} \Pi$ inductively in the obvious way:

$$
\begin{aligned}
{[\neg \varphi] } & =\neg[\varphi] ; \\
{[\varphi \wedge \psi] } & =[\varphi] \wedge[\psi] ; \\
{[\mathrm{B} \varphi] } & =\square[\varphi] ; \\
{[\forall p \varphi] } & =\forall p[\varphi] .
\end{aligned}
$$

Proposition 3.2 For any $\varphi \in \mathscr{L} \Pi, \tilde{\theta}(\varphi)=[\varphi](\theta)$ for all $\theta \in V$.

Now we identify two properties of these functions from $V$ to $\mathscr{B}$ that are important to us. 
Definition 3.5 For any $f: V \rightarrow \mathscr{B}$, we say that $f$ is fixed if its range is $\operatorname{in} \operatorname{fp}(\mathscr{B})$ (that is, $\square f=f$ ); and we say that $f$ is local if for any $p \in$ Prop and $\theta \in V$,

$$
\text { if } b \leq a_{1} \leftrightarrow a_{2} \text {, then } b \leq f\left(\theta\left[a_{1} / p\right]\right) \leftrightarrow f\left(\theta\left[a_{2} / p\right]\right) \text {, }
$$

for all $b \in \mathrm{fp}(\mathscr{B})$ and $a_{1}, a_{2} \in \mathscr{B}$.

The intuition behind locality is that for $f$ to be local, what $f$ is below a fixpoint $b \in \mathrm{fp}(\mathscr{B})$ (namely what $f \wedge b$ is) should only depend on what the arguments are below $b$ (namely what $\theta(p) \wedge b$ is for all $p \in$ Prop). The above definition formalizes this intuition because in Boolean algebras, $x \leq y \leftrightarrow z$ iff $x \wedge y=x \wedge z$.

But why are these two properties important to us? Recall that what we are trying to show here is that $\forall p \mathrm{~B} \varphi \rightarrow \mathrm{B} \forall p \mathrm{~B} \varphi$ is valid on $\mathscr{B}$. If we can show that $\forall p p \mathrm{~B} \varphi$ ] is fixed, which means that $[\forall p \mathrm{~B} \varphi]=\square[\forall p \mathrm{~B} \varphi]$, then we are done. This is because, with $[\forall p \mathrm{~B} \varphi]$ being fixed, for any $\theta \in V$,

$$
\tilde{\theta}(\forall p \mathrm{~B} \varphi)=[\forall p \mathrm{~B} \varphi](\theta)=(\square[\forall p \mathrm{~B} \varphi])(\theta)=[\mathrm{B} \forall p \mathrm{~B} \varphi](\theta)=\tilde{\theta}(\mathrm{B} \forall p \mathrm{~B} \varphi) .
$$

Obviously, then, $\tilde{\theta}(\forall p \mathrm{~B} \varphi \rightarrow \mathrm{B} \forall p \mathrm{~B} \varphi)=\top$. So our goal is now reduced to showing that $[\forall p \mathrm{~B} \varphi]$ is fixed. Note that $[\forall p \mathrm{~B} \varphi]=\forall p \square[\varphi]$. It is trivial to see that $\square[\varphi]$ is fixed. So one might hope that we can show that whenever $f$ is fixed, $\forall p f$ is also fixed, and then claim victory. However, this is in general false, given the example we produced in Definition 3.2 above showing that the set of fixpoints $\mathrm{fp}(\mathscr{B})$ is in general not closed under arbitrary meets. One can construct an $f$ whose range (when we vary the $p$ coordinate of the input valuation) is precisely a set of fixpoints in $\mathrm{fp}(\mathscr{B})$ whose meet is not in $\mathrm{fp}(\mathscr{B})$. Then $\forall p f$ is not fixed. What is missing in this strategy of showing that $\forall p f$ is fixed whenever $f$ is fixed is precisely locality. We will show first that $[\mathrm{B} \varphi]$ must be local in addition to being fixed. We will then show that if $f$ is fixed and local, then $\forall p f$ is fixed and local.

To show that $[\mathrm{B} \varphi]$ is local, the following lemma, showing in fact that all definable functions are local, suffices.

Lemma 3.2 We have the following closure properties for local functions.

- Projection functions of the form $[p]$ for some $p \in$ Prop are local.

- Local functions are closed under Boolean combinations: if $f, g: V \rightarrow \mathscr{B}$ are local, then $f \wedge g$ and $\neg f$ are both local.

- Local functions are closed under $\square$.

- Local functions are closed under $\forall p$ for all $p \in$ Prop.

Hence, all definable functions are local.

Proof The first two points are easy. For the third, consider $\square f$ where $f$ is local. Then for a $b \in \mathrm{fp}(\mathscr{B})$, we need to show that

$$
\text { if } b \leq a_{1} \leftrightarrow a_{2} \text {, then } b \leq \square f\left(\theta\left[a_{1} / p\right]\right) \leftrightarrow \square f\left(\theta\left[a_{2} / p\right]\right) \text {. }
$$


Given that $f$ is local, when $b \leq a_{1} \leftrightarrow a_{2}, b \leq f\left(\theta\left[a_{1} / p\right]\right) \leftrightarrow f\left(\theta\left[a_{2} / p\right]\right)$. Box both sides (using that $\square$ is monotone), and we see that

$$
b=\square b \leq \square\left(f\left(\theta\left[a_{1} / p\right]\right) \leftrightarrow f\left(\theta\left[a_{2} / p\right]\right)\right) \leq \square f\left(\theta\left[a_{1} / p\right]\right) \leftrightarrow \square f\left(\theta\left[a_{2} / p\right]\right) .
$$

The first equality is due to that $b \in \mathrm{fp}(\mathscr{B})$, and the last inequality is by the normality of $\square$ : it commutes with finite meets, and $\square(x \rightarrow y) \leq \square x \rightarrow \square y$.

Now, for the fourth point, consider $\forall p f$ where $f$ is local. In this case, we need to work with an arbitrary $q \in$ Prop, an arbitrary $b \in \mathrm{fp}(\mathscr{B})$, and arbitrary $a_{1}, a_{2} \in \mathscr{B}$ such that $b \leq a_{1} \leftrightarrow a_{2}$, and show that

$$
b \leq \bigwedge\left\{f\left(\theta\left[a_{1} / q\right][c / p]\right) \mid c \in \mathscr{B}\right\} \leftrightarrow \bigwedge\left\{f\left(\theta\left[a_{2} / q\right][c / p]\right) \mid c \in \mathscr{B}\right\} .
$$

If $q=p$, then this is trivially true (the right-hand-side of the inequality is $\top$ ). So we now consider the case when $q \neq p$, in which case $\theta\left[a_{1} / q\right][c / p]=\theta[c / p]\left[a_{1} / q\right]$ and $\theta\left[a_{2} / q\right][c / p]=\theta[c / p]\left[a_{2} / q\right]$. For simplicity, let $\theta_{c}=\theta[c / p]$. Since we assumed that $f$ is local, by definition, we have the following for all $c \in \mathscr{B}$ :

$$
b \leq f\left(\theta_{c}\left[a_{1} / q\right]\right) \leftrightarrow f\left(\theta_{c}\left[a_{2} / q\right]\right) .
$$

Thus, $b \leq \bigwedge\left\{f\left(\theta_{c}\left[a_{1} / q\right]\right) \leftrightarrow f\left(\theta_{c}\left[a_{2} / q\right]\right) \mid c \in \mathscr{B}\right\}$. Hence, all we need now is the following simple principle on complete Boolean algebras:

$$
\begin{aligned}
\bigwedge\left\{f\left(\theta_{c}\left[a_{1} / q\right]\right) \leftrightarrow f\left(\theta_{c}\left[a_{2} / q\right]\right) \mid c \in \mathscr{B}\right\} & \leq \\
& \bigwedge\left\{f\left(\theta_{c}\left[a_{1} / q\right]\right) \mid c \in \mathscr{B}\right\} \leftrightarrow \bigwedge\left\{f\left(\theta_{c}\left[a_{2} / q\right]\right) \mid c \in \mathscr{B}\right\} .
\end{aligned}
$$

To see that in general, $\bigwedge_{i \in I}\left(x_{i} \leftrightarrow y_{i}\right) \leq \bigwedge_{i \in I} x_{i} \leftrightarrow \bigwedge_{i \in I} y_{i}$, note that it is enough to show that $\bigwedge_{i \in I}\left(x_{i} \leftrightarrow y_{i}\right) \wedge \bigwedge_{i \in I} x_{i} \leq \bigwedge_{i \in I} y_{i}$ and symmetrically that $\bigwedge_{i \in I} x_{i} \leftrightarrow y_{i} \wedge \bigwedge_{i \in I} y_{i} \leq \bigwedge_{i \in I} x_{i}$. Both of them are easy.

That $[\mathrm{B} \varphi]$ is fixed is immediate from the following lemma.

Lemma 3.3 We have the following closure properties for fixed functions.

- For any $f: V \rightarrow \mathscr{B}, \square f$ is fixed.

- Fixed functions are closed under Boolean combinations.

- Not all fixed functions are closed under $\forall p$.

Proof Immediate from Lemma 3.1.

The only missing piece then is the following lemma.

Lemma 3.4 Fixed local functions are closed under $\forall p$. That is, if $f$ is fixed and local, then $\forall p f$ is also fixed and local, for any $p \in$ Prop.

Proof Pick an arbitrary fixed and local $f: V \rightarrow \mathscr{B}$. Since fixed local functions are closed under Boolean combinations and local functions are closed under $\forall p$, without loss of generality, we only need to show that $\exists p f$ is also fixed.

The idea is the following. Pick an arbitrary $\theta \in V$. Then we show that $(\exists p f)(\theta)$ in fact has a witness: there exists $c \in \mathscr{B}$ such that $(\exists p f)(\theta)=f(\theta[c / p])$. Since $f$ is 
fixed, $f(\theta[c / p]) \in \mathrm{fp}(\mathscr{B})$. So, $(\exists p f)(\theta)$, being just $f(\theta[c / p])$, is in $\mathrm{fp}(\mathscr{B})$. Since $\theta$ is arbitrarily chosen, this shows that $\exists p f$ is fixed. As a consequence, $\square \exists p f=\exists p f$.

Hence, let us fix an arbitrary $\theta \in V$ and from now on write $f(a)$ for $f(\theta[a / p])$. Since $f$ is fixed and local, we know that:

- For any $a \in \mathscr{B}, f(a) \in \mathrm{fp}(\mathscr{B})$.

- For any $b \in \operatorname{fp}(\mathscr{B})$ and any $a, a^{\prime} \in \mathscr{B}$ such that $b \leq a \leftrightarrow a^{\prime}, b \leq f(a) \leftrightarrow f\left(a^{\prime}\right)$.

Our goal, then, is to show that there is a $c \in \mathscr{B}$ such that $f(c)=\bigvee\{f(a) \mid a \in \mathscr{B}\}$.

To this end, let $F=\{f(a) \mid a \in \mathscr{B}\}$. We will show soon that $F$ as a poset (with $\leq$ inherited from $\mathscr{B}$ ) has the following two properties:

- (Directed) For any $a, b \in F$, there is a $c \in F$ such that $a, b \leq c$.

- (Chain) For any ascending chain $\left\langle a_{i}\right\rangle_{i \leq \kappa}$ in $F$, there is $t \in F$ such that for all $i<\kappa, a_{i} \leq t$.

From these two conditions, it is easy to see that $F$ has an $x$ that is the greatest in $F$. By Zorn's lemma, $F$ has a maximal element. By (Directed), the maximal element given by Zorn's lemma must also be the greatest element of $F$. Hence, the join of $F$ is in $F$. Then, anything in $f^{-1}(\bigvee F)$ can serve as the witness for $(\exists p f)(\theta)=\bigvee F$.

Now we show the two properties. For (Directed), note that $F \subseteq \mathrm{fp}(\mathscr{B})$ since $f$ is fixed. Thus, if we pick $b_{1}, b_{2} \in F$ and $a_{1}, a_{2} \in \mathscr{B}$ such that $f\left(a_{1}\right)=b_{1}$ and $f\left(a_{2}\right)=$ $b_{2}$, we can apply locality here. Indeed, let $a=\left(a_{1} \wedge b_{1}\right) \vee\left(a_{2} \wedge\left(b_{2} \backslash b_{1}\right)\right)$. Note that $b_{2} \backslash b_{1} \in \mathrm{fp}(\mathscr{B})$. It is also easy to see that $b_{1} \leq a \leftrightarrow a_{1}$ and $b_{2} \backslash b_{1} \leq a \leftrightarrow a_{2}$. Then, $b_{1} \leq f(a) \leftrightarrow f\left(a_{1}\right)$ and $b_{2} \backslash b_{1} \leq f(a) \leftrightarrow f\left(a_{2}\right)$. However, by the way we picked $a_{1}$ and $a_{2}, b_{1} \leq f\left(a_{1}\right)$ and $b_{2} \backslash b_{1} \leq f\left(a_{2}\right)$. Thus, $b_{1} \leq f(a)$, and $b_{2} \backslash b_{1} \leq f(a)$, and $b_{1} \vee b_{2} \leq f(a) \in F$.

For (Chain), we can use the same strategy. Pick an ascending chain $\left\langle b_{i}\right\rangle_{i \leq \kappa}$ in $F$ for some cardinal $\kappa$ with a corresponding sequence $\left\langle a_{i}\right\rangle_{i<\kappa}$ such that $f\left(a_{i}\right)=b_{i}$ for all $i<\kappa$. Then inductively define $\left\langle c_{i}\right\rangle_{i<\kappa}$ by

$$
\begin{aligned}
c_{0} & =a_{0} \wedge b_{0} ; \\
c_{i+1} & =c_{i} \vee\left(a_{i+1} \wedge\left(b_{i+1} \backslash b_{i}\right)\right) ; \\
c_{\lambda} & =\left(\bigvee_{i<\lambda} c_{i}\right) \vee\left(a_{\lambda} \wedge\left(b_{\lambda} \backslash f\left(\bigvee_{i<\lambda} c_{i}\right)\right)\right) .
\end{aligned}
$$

By an easy induction, we can see that for all $i<\kappa, c_{i} \leq b_{i}$, (note that $\left(a_{\lambda} \wedge\left(b_{\lambda} \backslash f\right.\right.$ $\left.\left.\left.\left(\bigvee_{i<\lambda} c_{i}\right)\right)\right) \leq b_{\lambda}\right)$ and that $\left\langle c_{i}\right\rangle_{i<k}$ is an ascending chain). Less easy is the following:

1. For all $\lambda \leq \kappa, \bigvee_{i<\lambda} b_{i} \leq f\left(\bigvee_{i<\lambda} c_{i}\right)$.

We use strong induction here, and the base case is trivial. Now suppose as (IH) that for all $\delta<\lambda, \bigvee_{i<\delta} b_{i} \leq f\left(\bigvee_{i<\delta} c_{i}\right)$. Then our only goal is to show that $\bigvee_{i<\lambda} b_{i} \leq f\left(\bigvee_{i<\lambda} c_{i}\right)$

- Say $\lambda=\alpha+1$. Then $\bigvee_{i<\lambda} b_{i}=b_{\alpha}$ and $\bigvee_{i<\lambda} c_{i}=c_{\alpha}$ since $\left\langle b_{i}\right\rangle_{i<\kappa}$ and $\left\langle c_{i}\right\rangle_{i<\kappa}$ are both ascending chains. Hence we are just showing that $b_{\alpha} \leq f\left(c_{\alpha}\right)$. Now there are two cases.

- Say $\alpha=\beta+1$. Then $c_{\alpha}=c_{\beta} \vee\left(a_{\alpha} \wedge\left(b_{\alpha} \backslash b_{\beta}\right)\right)$. By (IH) applied to $\alpha$, $b_{\beta}=\bigvee_{i<\alpha} b_{i} \leq f\left(\bigvee_{i<\alpha} c_{i}\right)=f\left(c_{\beta}\right)$. Note also that $b_{\beta} \leq c_{\beta} \leftrightarrow c_{\alpha}$ 
and $b_{\alpha} \backslash b_{\beta} \leq a_{\alpha} \leftrightarrow c_{\alpha}$. By locality, then, $b_{\beta} \leq f\left(c_{\beta}\right) \leftrightarrow f\left(c_{\alpha}\right)$ and $b_{\alpha} \backslash b_{\beta} \leq f\left(a_{\alpha}\right) \leftrightarrow f\left(c_{\alpha}\right)$. However, by (IH) and the way we picked $a_{\alpha}, b_{\beta} \leq f\left(c_{\beta}\right)$ and $b_{\alpha} \backslash b_{\beta} \leq b_{\alpha} \leq f\left(a_{\alpha}\right)$. Thus $b_{\beta} \leq f\left(c_{\alpha}\right)$ and $b_{\alpha} \backslash b_{\beta} \leq f\left(c_{\alpha}\right)$. Thus $b_{\alpha} \leq f\left(c_{\alpha}\right)$.

- Say $\alpha$ is a limit ordinal. For convenience let $c_{\beta}=\bigvee_{i<\alpha} c_{i}$ and $b_{\beta}=$ $f\left(c_{\beta}\right)$. Here $b_{\beta} \in \mathrm{fp}(\mathscr{B})$ since $f$ is fixed. By definition, $c_{\alpha}=c_{\beta} \vee$ $\left(a_{\alpha} \wedge\left(b_{\alpha} \backslash b_{\beta}\right)\right)$. Now we can apply the same strategy again to show that $b_{\alpha} \leq f\left(c_{\alpha}\right)$.

- Then we consider the interesting case where $\lambda$ is a limit ordinal. What we need to show here is that $\bigvee_{i<\lambda} b_{i} \leq f\left(\bigvee_{i<\lambda} c_{i}\right)$, which means for all $j<\lambda, b_{j} \leq$ $f\left(\bigvee_{i<\lambda} c_{i}\right)$. To show this, pick an arbitrary $j<\lambda$. Then consider $b_{j} \wedge \bigvee_{i<\lambda} c_{i}$. Here we claim that this is just $c_{j}$. First, $b_{j} \wedge \bigvee_{i<\lambda} c_{i}=\bigvee_{i<\lambda}\left(b_{j} \wedge c_{i}\right)$. (This distributivity law holds on any complete Boolean algebra.) Also, for $i \leq j, b_{j} \wedge$ $c_{i}=c_{i}$ since $c_{i} \leq b_{i}$ for all $i<\kappa$. Since $\left\langle c_{i}\right\rangle_{i<\kappa}$ is ascending, $\bigvee_{i \leq j}\left(b_{j} \wedge c_{i}\right)=$ $c_{j}$. Thus we only need to show that for all $i$ such that $j \leq i<\lambda, b_{j} \wedge c_{i}=c_{j}$. Obviously we need to do this by induction. The base case where $i=j$ is trivial (again, by $c_{i} \leq b_{i}$ ). For the inductive step:

$-\quad b_{j} \wedge c_{i+1}=\left(b_{j} \wedge c_{i}\right) \vee\left(b_{j} \wedge a_{i+1} \wedge\left(b_{i+1} \backslash b_{i}\right)\right)=\left(b_{j} \wedge c_{i}\right) \vee\left(a_{i+1} \wedge b_{i+1} \wedge\right.$ $\left.\neg b_{i} \wedge b_{j}\right)=b_{j} \wedge c_{i}=c_{j}$. Here the first equality is by distributivity, the second by simple Boolean reasoning, the third by the fact that $j<i$ and hence $b_{j} \leq b_{i}$ and $b_{j} \wedge \neg b_{i}=\perp$, and the fourth by the induction hypothesis.

- For a limit ordinal $k$ between $j$ and $\lambda, b_{j} \wedge c_{k}=\left(b_{j} \wedge \bigvee_{i<k} c_{i}\right) \vee\left(b_{j} \wedge\right.$ $\left.a_{k} \wedge\left(b_{k} \backslash f\left(\bigvee_{i<k} c_{i}\right)\right)\right)$. Now, by the induction hypothesis that for all $i$ such that $j \leq i<k, b_{j} \wedge c_{i}=c_{j}$, we get that $b_{j} \wedge \bigvee_{i<k} c_{i}=c_{j}$. Recall that we are inside another induction with (IH) assumed. Applying (IH) to $k$, we get that $b_{j} \leq \bigvee_{i<k} b_{i} \leq f\left(\bigvee_{i<k} c_{i}\right)$. Hence, $b_{j} \wedge a_{k} \wedge\left(b_{k} \backslash\right.$ $\left.f\left(\bigvee_{i<k} c_{i}\right)\right)=\perp$, and $b_{j} \wedge c_{k}=c_{j}$.

So, we have shown that $b_{j} \wedge \bigvee_{i<\lambda} c_{i}=c_{j}$. Adding this to the fact that $c_{j} \leq b_{j}$, $b_{j} \leq \bigvee_{i<\lambda} c_{i} \leftrightarrow c_{j}$. By locality, $b_{j} \leq f\left(\bigvee_{i<\lambda} c_{i}\right) \leftrightarrow f\left(c_{j}\right)$. But $b_{j} \leq f\left(c_{j}\right)$ since we can apply (IH) to $j+1$. Thus, $b_{j} \leq f\left(\bigvee_{i<\lambda} c_{i}\right)$, and we are done here.

Now we put the three lemmas together to prove the main theorem of this section.

Theorem 3.1 For any $\varphi \in \mathscr{L} \Pi$ and any complete $K D 45$ algebra $\mathscr{B}, 4^{\forall}=\forall p \mathrm{~B} \varphi \rightarrow$ $\mathrm{B} \forall p \square \varphi$ is valid on $\mathscr{B}$.

Proof Let $\mathscr{B}$ be an arbitrary complete KD45 algebra. Then all the definitions, propositions, and lemmas in this section apply to $\mathscr{B}$. A straightforward induction shows that $[\mathrm{B} \varphi]$ as defined in Definition 3.4 is a definable function from $V$ to $\mathscr{B}$ according to Definition 3.3. By Lemma 3.2, it is local. Since $[\mathrm{B} \varphi]=\square[\varphi]$, by Lemma 3.3, it is fixed. Thus, by Lemma 3.4, $[\forall p \mathrm{~B} \varphi]=\forall p[\mathrm{~B} \varphi]$ is also fixed as $[\mathrm{B} \varphi]$ is both fixed and local. Thus, $[\forall p \mathrm{~B} \varphi]=\square[\forall p \mathrm{~B} \varphi]=[\mathrm{B} \forall p \mathrm{~B} \varphi]$, and hence 
$[\forall p \mathrm{~B} \varphi \rightarrow \mathrm{B} \forall p \mathrm{~B} \varphi]=[\forall p \mathrm{~B} \varphi] \rightarrow[\mathrm{B} \forall p \mathrm{~B} \varphi]=[\forall p \mathrm{~B} \varphi] \rightarrow[\forall p \mathrm{~B} \varphi]$, which is constantly $\top$ since in any Boolean algebra and for any element $x$ in it, $x \rightarrow x$ is the top element. Then, according to Proposition 3.2, for any valuation $\theta$ on $\mathscr{B}$, $\tilde{\theta}\left(4^{\forall}\right)=\tilde{\theta}(\forall p \mathrm{~B} \varphi \rightarrow \mathrm{B} \forall p \mathrm{~B} \varphi)=\left[4^{\forall}\right](\theta)=\mathrm{T}$. Hence, $4^{\forall}$ is valid on $\mathscr{B}$.

Before we move on to the next section, let us briefly reflect on what this theorem tells us. First, we see that to separate $4^{\forall}$ from $\mathrm{KD} 45 \Pi$ using algebraic semantics, we need to drop the completeness assumption. The difficulty here is that it is not known which meets and joins we need to make the semantics for all formulas with propositional quantifiers well defined. One trivial way to get a KD45 algebra on which the semantics is well defined is to use the Lindenbaum algebra of KD45П. However, showing that $4^{\forall}$ in this algebra does not evaluate to $T$ is plainly equivalent to showing that KD45 $\Pi$ does not prove $4^{\forall}$, so this is hardly making any progress. What we need here is a less abstract way to build KD45 algebras on which the semantics is well defined, and this seems to require a less abstract sufficient condition for the well-definedness of the semantics that is strictly weaker than lattice completeness. Another way is to use the semantics with propositional contingency as we sketched at the end of Section 2. The difficulty there seems to be validating the $\Pi$-principles.

Second, we mentioned in Lemma 3.1s that there is a complete KD45 algebra $\mathscr{B}$ such that $\mathrm{fp}(\mathscr{B})$ is not closed under arbitrary meets in $\mathscr{B}$. If we examine the syntactical structure of $4^{\forall}=\forall p \mathrm{~B} \varphi \rightarrow \mathrm{B} \forall p \mathrm{~B} \varphi$, it seems that $4^{\forall}$ is a candidate formula whose validity on a $\mathrm{KD} 45$ algebra $\mathscr{B}$ would correspond to the condition that $\mathrm{fp}(\mathscr{B})$ is closed under arbitrary meets. After all, as we vary the valuation of $p, \mathrm{~B} \varphi$ picks up a subset of $\mathrm{fp}(\mathscr{B})$. Then the validity of $4^{\forall}$ says that this meet is below ${ }^{6}$ the $\square$ of this meet, which then implies, with a bit of manipulation like what we did in Lemma 3.1, that this meet is also a fixpoint. Of course, this is not to be: while the validity of $4^{\forall}$ entails that some meets of fixpoints are still fixpoints, it does not correspond to the condition that the set of fixpoints is closed under arbitrary meets. What is at work here is that the expressivity constraint of the language is also a constraint on which sets of fixpoints $\mathrm{B} \varphi$ can pick up.

Can there be a way to syntactically capture the condition that the set of fixpoints is closed under arbitrary meets? One way is to add uninterpreted propositional operators. For example, let $\mathscr{L} \Pi O$ be the language extending $\mathscr{L} \Pi$ with a unary operator $O$. Then, a valuation $\theta$ assigns not only an element in $\mathscr{B}$ to each propositional variable, but also a unary function on $\mathscr{B}$ to $O$. The semantics of the formulas in $\mathscr{L} \Pi O$ under valuation $\theta$ can be defined in the obvious way. Then, the validity of $\forall p \mathrm{~B} O p \rightarrow \mathrm{B} \forall p \mathrm{~B} O p$ on $\mathscr{B}$ corresponds to $\mathrm{fp}(\mathscr{B})$ being closed under arbitrary meets. We leave further investigation of this formalism as future work.

\section{Completeness of $\mathrm{KD} 4{ }^{\forall} 5 \Pi$ with Respect to Complete Proper Filter Algebras}

In this section, we show the following completeness theorem.

\footnotetext{
${ }^{6}$ Through out this paper, we use "below" in the weak sense when talking about elements in lattices.
} 
Theorem 4.1 For any $\varphi \in \mathscr{L} \Pi$, if $\varphi$ is valid on all complete proper filter algebras, then $\varphi \in \mathrm{KD} 4{ }^{\forall} 5 \Pi$.

Like most completeness proofs, we can show instead that any non-theorem $\varphi$ of $\mathrm{KD} 4^{\forall} 5 \Pi$ is refuted by a complete proper filter algebra. Then, one strategy particularly suitable for algebraic semantics, also used in [40], is the following:

- Construct the Lindenbaum-Tarski algebra $\mathscr{B}$ of $\mathrm{KD} 4{ }^{\forall} 5 \Pi$. Automatically, $\mathscr{B}$ is a KD45 algebra, and $\varphi$ is evaluated to a non-top element in $\mathscr{B}$ by a naturally defined valuation.

- Transform $\mathscr{B}$ into a complete proper filter algebra $\mathscr{C}$ while keeping $\varphi$ evaluated to a non-top element. The transformation is typically by first turning $\mathscr{B}$ into a proper filter algebra and then using a construction like MacNeille completion.

The problem with this approach is that neither of the standard methods of constructing MacNeille completions of Boolean algebra with operators, namely the lower and upper MacNeille completions (see [46] or [47]), can be used here. Since proper filter algebras correspond to well-connected KD45 algebras, we can directly use the definition of lower or upper MacNeille completion. However, the upper MacNeille completion of a well-connected KD45 algebra is not necessarily a KD45 algebra (we leave this to the reader; the proper filter agebra based on the finite-cofinite algebra in the proof of Proposition 4.1 below can be used to show this as well). The lower MacNeille completion construction does preserve the property of being well-connected and KD45. When translated to proper filter algebras, we get the following definition.

Definition 4.1 The lower MacNeille completion $\overline{\mathscr{B}}$ of a proper filter algebra $\mathscr{B}=$ $\langle B, F\rangle$ is $\langle\bar{B}, \uparrow F\rangle$ where $\bar{B}$ is the MacNeille completion of $B$ (which can be assumed to have $B$ as a subalgebra) and $\uparrow F=\{a \in \bar{B} \mid \exists b \in F, b \leq \bar{B} a\}$.

Intuitively, the lower MacNeille completion of a proper filter algebra is obtained by first completing the Boolean algebra part and then extending the original filter minimally to be a filter in the completed Boolean algebra. The problem with the lower MacNeille completion construction is that it may change the semantic value of a sentence from a non-top element to the top element. This means that invalidity, or equivalently refutability, is not preserved.

Proposition 4.1 There is a sentence $\varphi \in \mathscr{L} \Pi$ and a proper filter algebra $\mathscr{B}$ such that $\varphi$ is invalid in $\mathscr{B}$ but is valid in the lower MacNeille completion $\overline{\mathscr{B}}$.

Proof Let $B$ be the Boolean algebra of finite or cofinite subsets of $\mathbb{N}$ and $F$ the set of cofinite sets of $\mathbb{N}$. Then $\mathscr{B}=\langle B, F\rangle$ is a proper filter algebra. The lower MacNeille completion $\overline{\mathscr{B}}$ is then $\langle\wp(\mathbb{N}), F\rangle$ where $\wp(\mathbb{N})$ is the powerset algebra of $\mathbb{N}$. Note that, importantly, the lower MacNeille completion does not change $F$.

Let $\varphi$ be $\exists p(\neg \mathrm{B} p \wedge \neg \mathrm{B} \neg p)$. For any valuation $\theta$ on $\mathscr{B}, \tilde{\theta}(\varphi)$ is $\perp$, since $\tilde{\theta}(p) \in B$ is either finite or cofinite, and thus either $\tilde{\theta}(p)$ or $\tilde{\theta}(\neg p)$ is cofinite. Then either $\tilde{\theta}(\neg \mathrm{B} p)$ or $\tilde{\theta}(\neg \mathrm{B} \neg p)$ is $\perp$. This means that $\tilde{\theta}(\varphi)=\perp$. Now, let $\theta$ be a valuation 
on $\overline{\mathscr{B}}$ such that $\theta(p)$ is the set of even numbers. Then $\theta(p)$ is infinite and coinfinite. Hence $\tilde{\theta}(\neg \mathrm{B} p \wedge \neg \mathrm{B} \neg p)$ is $\top$, and so $\tilde{\theta}(\varphi)=\top$. Since $\varphi$ is a sentence with no free variables, this means that $\varphi$ is valid on $\overline{\mathscr{B}}$.

The lesson from the above example is that, to preserve the invalidity of $\exists p(\neg \mathrm{B} p \wedge$ $\neg \mathrm{B} \neg p$ ), we need to extend $F$, the filter of cofinite sets, into an ultrafilter. However, there seems to be no canonical way to do this: every ultrafilter seems as good as any other. It seems that to prove the completeness of $\mathrm{KD} 4^{\forall} 5 \Pi$, we need a more detailed analysis of both the algebras and the system itself.

Our approach is based on the following observation: every complete proper filter algebra $\mathscr{B}=\langle B, F\rangle$ has a natural quotient Boolean algebra $B / F$, and a belief formula in the form of $\mathrm{B} \varphi$, where $\varphi$ contains only Boolean connectives, is asserting that $\varphi$ evaluates to the top element in the quotient $B / F$. Hence, $\mathrm{B} \varphi$ is talking about the quotient algebra $B / F$. With propositional quantifiers, $\mathscr{L} \Pi$ can in fact talk about $B / F$ in a first-order way.

Given this observation, we have the following strategy, where we focus on just the sentences since for any formula, it is valid if and only if its universal closure, the result of bounding all free variables with universal quantifiers, is valid:

- For a sentence $\varphi$ in $\mathscr{L} \Pi$ that is valid in all complete proper filter algebras, find a corresponding $\psi$ in a first-order language for Boolean algebras. This $\psi$ will be valid on all natural quotient Boolean algebras of all complete proper filter algebras.

- Show that if a first-order sentence is valid on all natural quotient Boolean algebras of complete proper filter algebras, then it is in fact valid on all non-trivial Boolean algerbas. Consequently, $\psi$ will have a proof in the usual first-order logic for all non-trivial Boolean algebras.

- Translate the first-order proof of $\psi$ into a proof of $\varphi$ in $\mathrm{KD} 4{ }^{\forall} 5 \Pi$.

The main difficulties of implementing this strategy lie in the first two steps. First, in fact, not every sentence $\varphi \in \mathscr{L} \Pi$ can be translated into a first-order sentence to be evaluated on the natural quotient Boolean algebras. The reason is that a sentence in $\mathscr{L} \Pi$ can be evaluated to a proposition that is neither top nor bottom, yet a first-order sentence can only be true or false. To cope with this, several auxiliary languages will be introduced, so that we can separate the translatable part and the non-translatable part of a sentence in $\mathscr{L} \Pi$. It turns out that the non-translatable part is well behaved, so we can proceed with them.

In the second step, a theorem about the natural quotients of complete proper filter algebras is needed. Essentially, it has to be shown that the non-trivial quotients of complete Boolean algebras are general enough to validate only those first-order formulas that are valid in all non-trivial Boolean algebras. In other words, the first-order logic of the non-trivial quotients of complete Boolean algebras is precisely the firstorder logic of all non-trivial Boolean algebras. While the first-order logic remains the same, it should be mentioned here that there are interesting special properties of quotients of complete Boolean algebras, e.g., countable separation property (see [48], 
Lemma 5.27). Previous results about the quotients of complete Boolean algebras include [49, 50], and [51]. We will extend the result in [51] to fulfill our purpose.

The rest of this section is split into four subsections. In Section 4.1, we define a number of auxiliary languages, their semantics, and translations between some of them. In Section 4.2, we show how the completeness of $\mathrm{KD} 4{ }^{\forall} 5 \Pi$ follows from two lemmas resolving the two difficulties mentioned above. Then, the next two subsections, Sections 4.3 and 4.4, are devoted to the two lemmas, respectively.

\subsection{Auxiliary languages, semantics, and translations}

Definition 4.2 Let $\mathscr{L} \Pi z g$ denote the language extending $\mathscr{L} \Pi$ with two new propositional constants $z$ and $g$. For more readability, we sometimes use overline instead $\neg$ for the negation of formulas and omit the $\wedge$ in a conjunction of literals. Now we define the following languages:

$\begin{array}{llr}\mathscr{L} \text { Bool }: t:=p|\top| \neg t \mid(t \wedge t) & \text { where } p \in \text { Prop; } \\ \mathscr{L} \text { Boolzg }: \varphi::=\mathrm{z}|\mathrm{g}| p|\top| \neg \varphi \mid(\varphi \wedge \varphi) & \text { where } p \in \text { Prop; } \\ \mathscr{L} \text { B } 1 \text { zg }: \varphi::=\mathrm{B} t|\neg \varphi|(\varphi \wedge \varphi) \mid \forall p \varphi & \text { where } t \in \mathscr{L} \text { Boolzg, } p \in \text { Prop. }\end{array}$

Very roughly speaking, $\mathrm{z}$ and $\mathrm{g}$ will be used to capture the non-translatable part, and $\mathscr{L}$ В $\Pi z g$ will be the translatable part. Recall that the main difficulty of translating $\mathscr{L} \Pi$ to a first-order logic is that there are sentences in $\mathscr{L} \Pi$ that evaluate to some intermediate proposition in some complete proper filter algebra. We will effectively show later that the Boolean combinations of $z$ and $g$ exhaust all possible semantic values that a sentence in $\mathscr{L} \Pi$ can take, and eventually every formula in $\mathscr{L} \Pi$ is provably equivalent to a Boolean combination of $\mathrm{z}, \mathrm{g}$, and formulas in $\mathscr{L} \mathrm{B} \Pi z \mathrm{z}$. It will be shown later in this section that $\mathscr{L} \mathrm{B} \Pi z \mathrm{z}$ is translatable to a first-order language.

The next definition fixes the interpretation of the new constants $z$ and $g$.

Definition 4.3 For any complete proper filter algebra $\mathscr{B}=\langle B, F\rangle$, define $z_{\mathscr{B}}, g_{\mathscr{B}}$ by

$$
\begin{aligned}
z_{\mathscr{B}} & =\bigwedge F \\
g_{\mathscr{B}} & =\bigvee\left\{a \in \mathscr{B} \mid a \text { is an atom and } a \leq z_{\mathscr{B}}\right\}
\end{aligned}
$$

The subscripts of $z_{\mathscr{B}}$ and $g_{\mathscr{B}}$ may be dropped if the context of which algebra we are talking about is clear.

For any valuation $\theta$ on $\mathscr{B}$, we then extend it uniquely to a $\mathscr{L} \Pi z g$-valuation $\tilde{\theta}$ : $\mathscr{L}$ חzg $\rightarrow B$, using the definition in Definition 2.5 plus two more clauses for $z$ and $\mathrm{g}: \tilde{\theta}(\mathrm{z})=z$ and $\tilde{\theta}(\mathrm{g})=g$.

Under this semantics, $g$ and $z g$ are semantically equivalent, but $\bar{g}$ and $z \bar{g}$ are not. For symmetry, we will mostly use zg instead of $g$ to contrast $z \bar{g}$.

It is important to see that for every complete proper filter algebra $\mathscr{B}, z_{\mathscr{B}}$ and $g_{\mathscr{B}}$ are expressible in $\mathscr{L} \Pi$. For this, we introduce a few more abbreviations. 
Definition 4.4 Define the following abbreviations:

$$
\begin{aligned}
\langle\mathrm{z}\rangle \varphi & =\widehat{\mathrm{B}}(\mathrm{z} \wedge \varphi) ; \\
{[\mathrm{z}] \varphi } & =\mathrm{B}(\mathrm{z} \rightarrow \varphi) ; \\
\operatorname{at}(\varphi) & =\langle\mathrm{z}\rangle \varphi \wedge \forall q([\mathrm{z}](\varphi \rightarrow q) \vee[\mathrm{z}](\varphi \rightarrow \neg q)) .
\end{aligned}
$$

Here $q$ is some variable not free in $\varphi$.

Proposition 4.2 For any complete proper filter algebra $\mathscr{B}$ and valuation $\theta$ :

1. $\tilde{\theta}(\langle z\rangle \varphi)=\tilde{\theta}(\neg[z] \neg \varphi)$.

2. $\tilde{\theta}(\langle z\rangle \varphi)$ and $\tilde{\theta}([\mathrm{z}] \varphi)$ are either $\top$ or $\perp$.

3. $\tilde{\theta}(\langle z\rangle \varphi)=\top$ if and only if $z \wedge \tilde{\theta}(\varphi)$ is not $\perp$.

4. $\tilde{\theta}([z] \varphi)=\top$ if and only if $\tilde{\theta}(\varphi) \geq z$. In other words, $\tilde{\theta}([\mathrm{z}] \varphi)=\top$ if and only if $\tilde{\theta}(\varphi) \wedge z=z$.

5. $\tilde{\theta}(a t(\varphi))=\tilde{\theta}(\langle z\rangle \varphi \wedge \neg \exists q(\langle z\rangle(\varphi \wedge q) \wedge\langle z\rangle(\varphi \wedge \neg q)))$ where $q$ is not free in $\varphi$.

6. $\tilde{\theta}($ at $(\varphi))$ is either $\top$ or $\perp$. It is the former if and only if $z \wedge \tilde{\theta}(\varphi)$ is an atom in (the Boolean algebra part of) $\mathscr{B}$.

7. $z=\tilde{\theta}(\forall p(\mathrm{~B} p \rightarrow p))$.

8. $g=\tilde{\theta}(\forall p(\mathrm{~B} p \rightarrow p) \wedge \exists p(p \wedge a t(p))$.

Proof The last item may need some explanation. The rest are straightforward. To start, unpack the semantics of $\tilde{\theta}(\forall p(\mathrm{~B} p \rightarrow p) \wedge \exists p(p \wedge a t(p)))$, and we see that it is

$$
\begin{aligned}
& \tilde{\theta}(\forall p(\mathrm{~B} p \rightarrow p)) \wedge \bigvee\{\widehat{\forall[a / p]}(p \wedge a t(p)) \mid a \in \mathscr{B}\} \\
&=z \wedge \bigvee\{a \wedge \widehat{\theta[a / p]}(a t(p)) \mid a \in \mathscr{B}\} \\
&=\bigvee\{z \wedge a \wedge \widehat{\theta[a / p]}(a t(p)) \mid a \in \mathscr{B}\} .
\end{aligned}
$$

Now if $a$ is an atom below $z$, then $z \wedge a=a$, which is still an atom. Then, given that $\widehat{\theta[a / p]}=a$ and hence $z \wedge \widehat{\theta[a / p]}=z \wedge a, \widehat{\theta[a / p]}(a t(p))=\top$ according to item 6 above. Then $z \wedge a \wedge \widehat{\theta[a / p]}(a t(p))=a$. This means that all atoms below $z$ are included in the join. On the other hand, if $a$ is not an atom below $z$, then $z \wedge a \wedge$ $\widehat{\theta[a / p]}(a t(p))=\perp$ since $\widehat{\theta[a / p]}(a t(p))$ is $\perp$, again by item 6 above. Thus the join is precisely the join of atoms below $z$.

It can also be shown that $\exists p(p \wedge a t(p))$ expresses the join of those elements whose meet with $z$ is an atom. However, taking this as a primitive seems to be less convenient for later work.

Now we focus on the fragment $\mathscr{L} \mathrm{B} \Pi z \mathrm{zg}$ and show in what sense it can be seen as talking about the natural quotients of complete proper filter algebras in a first-order way. To this end, we first define precisely what we mean by the natural quotient of a complete proper filter algebra $\mathscr{B}$.

Definition 4.5 For any complete proper filter algebra $\mathscr{B}=\langle B, F\rangle$, define its natural quotient $\mathscr{B} / F$ as the tuple $\left\langle B / F, \pi_{\mathscr{B}}(z), \pi_{\mathscr{B}}(g)\right\rangle$, where $\pi_{\mathscr{B}}$ is the quotient map from $B$ to $B / F$. We may drop the subscript of $\pi_{\mathscr{B}}$ when its context is clear. 
When viewed as a first-order structure, natural quotients of complete proper filter algebras are in the type of Boolean algebras augmented with two constants. Hence we define the following first-order language.

Definition 4.6 Let $\mathscr{F} \mathscr{O L}$ be the first-order language defined as below.

$$
\begin{array}{lr}
\text { Terms }: t:=p|\top| \neg t \mid(t \wedge t) & \text { where } p \in \text { Prop, } \\
\mathscr{F} \mathscr{O L}: \varphi::=\left(t=t^{\prime}\right)|\neg \varphi|(\varphi \wedge \varphi) \mid \forall p \varphi & \text { where } t, t^{\prime} \in \text { Terms. }
\end{array}
$$

Let $\mathscr{F} \mathscr{O L}$ zg be the first-order language extending $\mathscr{F} \mathscr{O L}$ by adding $\mathrm{z}$ and $\mathrm{g}$ as two constants.

Note that here we are intentionally reusing the symbols in $\mathscr{L} \Pi$, so that the translation between $\mathscr{F} \mathscr{O} \mathscr{L}$ zg and $\mathscr{L}$ B zg can be defined more directly. Note also that because we use the same symbols for meet in terms and conjunction in formulas, we need to bracket atomic formulas to avoid ambiguity. Now we define the standard first-order semantics for $\mathscr{F} \mathscr{O} \mathscr{L}$ zg.

Definition 4.7 For any triple $\langle B, z, g\rangle$ where $B$ is a Boolean algebra and $z, g \in B$, a variable assignment $\theta$ : Prop $\rightarrow B$ can be extended uniquely to $\tilde{\theta}$ on the terms of $\mathscr{F} \mathscr{O} \mathscr{L}$ zg by the following inductive clauses:

$$
\begin{aligned}
& \tilde{\theta}(p)=\theta(p), \tilde{\theta}(\mathrm{g})=g, \tilde{\theta}(\mathrm{z})=z, \tilde{\theta}(\top)=\top, \\
& \tilde{\theta}(\neg t)=\neg_{B} \tilde{\theta}(t), \tilde{\theta}((t \wedge s))=\tilde{\theta}(t) \wedge_{B} \tilde{\theta}(s) .
\end{aligned}
$$

Then the semantics of $\mathscr{F} \mathscr{O L} \mathrm{zg}$ is defined by

$$
\begin{aligned}
\langle B, z, g\rangle, \theta \vDash(t=s) & \Longleftrightarrow \tilde{\theta}(t)=\tilde{\theta}(s) \\
\langle B, z, g\rangle, \theta \vDash \neg \varphi & \Longleftrightarrow\langle B, z, g\rangle, \theta \not \varphi \\
\langle B, z, g\rangle, \theta \vDash(\varphi \wedge \psi) & \Longleftrightarrow\langle B, z, g\rangle, \theta \vDash \varphi \text { and }\langle B, z, g\rangle, \theta \vDash \psi \\
\langle B, z, g\rangle, \theta \vDash \forall p \varphi \quad & \Longleftrightarrow\langle B, z, g\rangle, \theta[a / p] \vDash \varphi \text { for all } a \in B .
\end{aligned}
$$

Syntactically, $\mathscr{L}$ B $\Pi z g$ and $\mathscr{F} \mathscr{O} \mathscr{L}$ zg are almost identical. This can be seen from how simply the translations between them can be defined.

Definition 4.8 Let $\mathscr{T}$ be the function from $\mathscr{L}$ B $\Pi z g$ to $\mathscr{F} \mathscr{O L}$ zg such that $\mathscr{T}(\varphi)$ is the result of replacing all occurrences in $\varphi$ of formulas of the form $\mathrm{B} \psi$ where $\psi \in \mathscr{L}$ Boolzg by $(\psi=\top)$.

Let $\mathscr{T}^{\prime}$ be the function from $\mathscr{F} \mathscr{O} \mathscr{L}$ zg to $\mathscr{L} \mathrm{B} \Pi z \mathrm{z}$ such that $\mathscr{T}^{\prime}(\varphi)$ is the result of replacing all atomic formulas $(s=t)$ by $\mathrm{B}(s \leftrightarrow t)$.

For example, recall the sentence $\exists p(\neg \mathrm{B} p \wedge \neg \mathrm{B} \neg p)$ we used in Proposition 4.1 where we showed that lower MacNeille completion does not preserve its semantic value. It is not hard to see that $\mathscr{T}(\exists p(\neg \mathrm{B} p \wedge \neg \mathrm{B} \neg p))=\exists p(\neg(p=\top) \wedge \neg(\neg p=$ $\top))$. Then $\mathscr{T}(\exists p(\neg \mathrm{B} p \wedge \neg \mathrm{B} \neg p))$ is false on a $\langle B, z, g\rangle$ iff $B$ is the 2-element Boolean algebra. This matches our observation there that $\exists p(\neg \mathrm{B} p \wedge \neg \mathrm{B} \neg p)$ is false (evaluates to $\perp$ ) on a complete proper filter algebra $\mathscr{B}=\langle B, F\rangle$ iff the filter $F$ is an ultrafilter, or equivalently iff the quotient $B / F$ is the 2-element Boolean algebra. 
To make the intuition that $\mathscr{L} \mathrm{B} \Pi z g$ talks about natural quotients in the first-order way precise, we use the following lemma.

Lemma 4.1 For any $\varphi \in \mathscr{L} \mathrm{B}$ Пzg, any complete proper filter algebra $\mathscr{B}=\langle B, F\rangle$, and any valuation $\theta$ on $\mathscr{B}, \tilde{\theta}(\varphi)$ is either $\top$ or $\perp$, and $\tilde{\theta}(\varphi)=\top$ if and only if $\langle B / F, \pi(z), \pi(g)\rangle, \pi \circ \theta \vDash \mathscr{T}(\varphi)$. As a corollary, when $\varphi$ is a sentence, $\mathscr{B} \vDash \varphi$ iff $\mathscr{B} / F_{\mathscr{B}} \vDash \mathscr{T}(\varphi)$.

Proof To avoid clutter, we omit the pair of parentheses immediately after $\pi$ and also the circle for composing $\pi$ with $\theta$. Hence $\pi p=\pi(p)$ and $\pi \theta(p)=\pi(\theta(p))=$ $(\pi \circ \theta)(p)$. Now obviously we need an induction on $\mathscr{L}$ В $\Pi z g$.

For any $\psi \in \mathscr{L}$ Boolzg, a simple induction shows that $\pi \tilde{\theta}(\psi)=\widetilde{\pi \theta}(\psi)$, since $\pi$ is a quotient map and hence a homomorphism. By our algebraic semantics defined in Definition 2.5, $\tilde{\theta}(\mathrm{B} \psi)$ is either $\top_{B}$ or $\perp_{B}$ according to whether $\tilde{\theta}(\psi)$ is in the filter $F$ or not. Also, since $F=\pi^{-1}\left(\top_{B / F}\right)$ by the definition of $\pi$ in Definition 4.5, $\pi \tilde{\theta}(\psi)=\top_{B / F}$ if and only if $\tilde{\theta}(\psi) \in F$. Then

$$
\begin{aligned}
\tilde{\theta}(\mathrm{B} \psi)=\top_{B} & \Longleftrightarrow \tilde{\theta}(\psi) \in F \Longleftrightarrow \tilde{\theta}(\psi)=\top_{B / F} \Longleftrightarrow \tilde{\pi}(\psi)=\top_{B / F} \\
& \Longleftrightarrow\langle B / F, \pi z, \pi g\rangle, \pi \theta \vDash \psi=\top \\
& \Longleftrightarrow\langle B / F, \pi z, \pi g\rangle, \pi \theta \vDash \mathscr{T}(\mathrm{B} \psi) .
\end{aligned}
$$

For formulas in $\mathscr{L} \mathrm{B} \Pi z \mathrm{zg}$ of the form $\neg \varphi$ where $\varphi \in \mathscr{L} \mathrm{B} \Pi z g$, note first that $\mathscr{T}(\neg \varphi)=\neg \mathscr{T}(\varphi)$. Also, $\tilde{\theta}(\neg \varphi)$ must be either $\top_{B}$ or $\perp_{B}$, given the induction hypothesis that $\tilde{\theta}(\varphi)$ is either $\top_{B}$ or $\perp_{B}$. Then, with the induction hypothesis that $\tilde{\theta}(\varphi)=\top_{B}$ iff $\langle B / F, \pi g, \pi z\rangle \vDash \mathscr{T}(\varphi)$, we have

$$
\begin{aligned}
\tilde{\theta}(\neg \varphi)=\top_{B} & \Longleftrightarrow \tilde{\theta}(\varphi)=\perp_{B} \Longleftrightarrow \tilde{\theta}(\varphi) \neq \top_{B} \\
& \Longleftrightarrow\langle B / F, \pi g, \pi z\rangle \not \mathscr{T}(\varphi) \\
& \Longleftrightarrow\langle B / F, \pi g, \pi z\rangle \vDash \neg \mathscr{T}(\varphi) \\
& \Longleftrightarrow\langle B / F, \pi g, \pi z\rangle \vDash \mathscr{T}(\neg \varphi) .
\end{aligned}
$$

For formulas in $\mathscr{L} \mathrm{B} \Pi z \mathrm{z}$ of the form $\varphi_{1} \wedge \varphi_{2}$, the situation is completely similar. We only need to do more replacements of equivalent claims coming from the induction hypothesis in this case.

Now consider $\forall q \varphi$. Note first that for all $a \in B$, by induction hypothesis we know that $\widetilde{\theta[a / q]}(\varphi)$ is either $\top_{B}$ or $\perp_{B}$, since the proof works for all valuations. Then, $\tilde{\theta}(\forall q \varphi)=\bigwedge\{\widehat{\theta[a / q]}(\varphi)\}$ must be either $\top_{B}$ or $\perp_{B}$. Moreover, being a meet of elements that are either $\top_{B}$ or $\perp_{B}$, it is $\top_{B}$ iff for all $a \in B, \widehat{\theta[a / q]}(\varphi)=\top_{B}$, which, using induction hypothesis, is equivalent to

$$
\text { for all } a \in B,\langle B / F, \pi g, \pi z\rangle, \pi \circ(\theta[a / q]) \vDash \mathscr{T}(\varphi) .
$$

On the other hand, according to the semantics, $\langle B / F, \pi g, \pi z\rangle, \pi \theta \vDash \mathscr{T}(\forall q \varphi)$ iff

$$
\text { for all } a \in B / F,\langle B / F, \pi g, \pi z\rangle,(\pi \circ \theta)[a / q] \vDash \mathscr{T}(\varphi) \text {. }
$$


Hence, all we need to show now is that Eqs. 1 and 2 are equivalent. Too see that they are equivalent, note that for any $a \in B, \pi \circ(\theta[a / q])$ is the same function as $(\pi \circ \theta)[\pi(a) / q]$. Then, given that $\pi$ is surjective, we are done.

\subsection{Logics in Auxiliary Languages and Completeness Proof}

In the last subsection, we introduced a number of fragments of $\mathscr{L}$ Пzg, including $\mathscr{L}$ B zg, which can be translated to the first-order language $\mathscr{F} \mathscr{O} \mathscr{L}$ zg in a semantically meaningful way: a formula $\varphi \in \mathscr{L}$ B zzg evaluates to $\top$ in a complete proper filter algebra $\mathscr{B}$ iff its translation $\mathscr{T}(\varphi)$ is true on the natural quotient $\mathscr{B} / F_{\mathscr{B}}$. From this we see that semantically, $\mathscr{L}$ B $\Pi z g$ is talking about the natural quotients of complete proper filter algebras in a first-order way.

In this subsection, we move to the logical side of this translation. We will first augment $\mathrm{KD} 4{ }^{\forall} 5 \Pi$ with two definitional axioms for the two new constants $\mathrm{z}$ and $\mathrm{g}$ and obtain $\mathrm{KD} 4{ }^{\forall} 5 \Pi \mathrm{zg}$. Then we provide a first-order logic FOLzg that is sound and complete with respect to a class of Boolean algebras with two extra named elements, which we call the class of zg-algebras. This class of zg-algebras is bigger than the class of natural quotients of complete proper filter algebras. However, we can show in a later section that the first-order logics of them are the same. In this section, the main task is to show that reasoning in FOLzg can be carried out in $\mathrm{KD} 4{ }^{\forall} 5 \Pi \mathrm{zg}$ by the reverse translation $\mathscr{T}^{\prime}$. Then, assuming that we can separate the translatable part $\mathscr{L}$ B zg and the non-translatable part and that FOLzg is not only the first-order logic of zg-algebras but also the first-order logic of the class of natural quotients of complete proper filter algebras, we show in this subsection that $\mathrm{KD} 4^{\forall} 5 \Pi \mathrm{zg}$ is complete with respect to all complete proper filter algebras. Given that $\mathrm{KD} 4{ }^{\forall} 5 \Pi z \mathrm{z}$ is a definitional extension of $\mathrm{KD} 4^{\forall} 5 \Pi$, the completeness of $\mathrm{KD} 4{ }^{\forall} 5 \Pi$ follows.

To start, we define the system $\mathrm{KD} 4{ }^{\forall} 5 \Pi \mathrm{zg}$.

Definition 4.9 Define logic $\mathrm{KD} 4{ }^{\forall} 5 \Pi \mathrm{zg}$ by extending $\mathrm{KD} 4{ }^{\forall} 5 \Pi$ with the following two axioms for $z$ and $g$ :

$$
\begin{aligned}
& \mathrm{z}: \mathrm{z} \leftrightarrow \forall p(\mathrm{~B} p \rightarrow p), \\
& \mathrm{g}: \mathrm{g} \leftrightarrow(\forall p(\mathrm{~B} p \rightarrow p) \wedge \exists p(p \wedge a t(p))) .
\end{aligned}
$$

The new axioms state the semantic definition of $z$ and $g$, as shown in Proposition 4.2. Moreover, the right-hand side of these two axioms are in $\mathscr{L} \Pi$. Hence $\mathrm{KD} 4{ }^{\forall} 5 \Pi \mathrm{zg}$ is a conservative extension of $\mathrm{KD} 4^{\forall} 5 \Pi$, and henceforth we only need to prove that $\mathrm{KD} 4{ }^{\forall} 5 \Pi \mathrm{zg}$ is the complete logic of complete proper filter algebras in $\mathscr{L} \Pi z \mathrm{z}$ according to the semantics defined in Definition 4.3.

Notation 1 In this and the next subsection, we will state many provability claims in the system $\mathrm{KD} 4{ }^{\forall} 5 \Pi z \mathrm{z}$. We write $\vdash \varphi$ for $\varphi$ being provable in $\mathrm{KD} 4{ }^{\forall} 5 \Pi \mathrm{zg}$ and write $\varphi \dashv \Vdash \psi$ for $\vdash \varphi \leftrightarrow \psi$. We treat $\dashv \vdash$ as a kind of equality between formulas so that in notation we chain them and use substitutions. We can do this because $\mathrm{KD} 4^{\forall} 5 \Pi \mathrm{zg}$ is a normal $\Pi$-logic, and thus $\dashv \Vdash$ is a congruence relation with respect to all connectives and quantifiers. 
Now we prove two lemmas that will be very useful. The first shows the importance of having $4^{\forall}$, and the second shows the use of the constant $z$. To state the first lemma, we call a formula $\varphi \in \mathscr{L}$ Пzg fully modalized when every propositional letter (those in Prop $\cup\{T, z, g\})$ is under the scope of some B. It is not hard to see that the fully modalized formulas in $\mathscr{L} \Pi z \mathrm{z}$ can be characterized by the grammar $\varphi::=\mathrm{B} \psi|\neg \varphi|$ $(\varphi \wedge \varphi) \mid \forall p \varphi$, where $\psi \in \mathscr{L}$ Пzg.

Lemma 4.2 For every fully modalized formula $\varphi \in \mathscr{L}$ Пzg, $\varphi \dashv \Vdash \mathrm{B} \varphi \dashv \Vdash \widehat{\mathrm{B}} \varphi$.

Proof First we show $\varphi \dashv \Vdash \mathrm{B} \varphi$ by induction.

- The case where $\varphi=\mathrm{B} \psi$ is trivial by $\mathrm{KD} 45$ since we are just showing that $\mathrm{B} \psi \dashv 1 \mathrm{BB} \psi$.

- Suppose $\varphi=\psi_{1} \wedge \psi_{2}$ where $\psi_{1}$ and $\psi$ are fully modalized. Then $\psi_{1} \dashv \vdash \mathrm{B} \psi_{1}$ and $\psi_{2} \dashv \vdash \mathrm{B} \psi_{2}$. Then $\psi_{1} \wedge \psi_{2} \dashv \vdash \mathrm{B} \psi_{1} \wedge \mathrm{B} \psi_{2} \dashv \vdash \mathrm{B}\left(\psi_{1} \wedge \psi_{2}\right)$.

- Suppose $\varphi=\neg \psi$ where $\psi$ is fully modalized, and hence $\psi \dashv \vdash \mathrm{B} \psi$. Then again by $\mathrm{KD} 45$, we have a chain of provable equivalences: $\neg \psi \dashv \vdash \neg \mathrm{B} \psi \dashv \vdash$ $\mathrm{B} \neg \mathrm{B} \psi \dashv \vdash \mathrm{B} \neg \psi$. The last equivalence can be obtained by simply replacing $\mathrm{B} \psi$ by $\psi$. Since $\mathrm{KD} 4{ }^{\forall} 5 \Pi \mathrm{zg}$ is normal, we can certainly do such replacements.

- Suppose $\varphi=\forall p \psi$ where $\psi$ is fully modalized and thus $\psi \dashv \vdash \mathrm{B} \psi$. Then $\forall p \psi \dashv \forall p \mathrm{~B} \psi$. By $4^{\forall}, \forall p \psi \dashv \vdash \mathrm{B} \forall p \mathrm{~B} \psi$. Then we can replace $\mathrm{B} \psi$ by $\psi$ again, and hence $\forall p \psi-1 \mathrm{~B} \forall p \varphi$.

Then for any fully modalized formula $\varphi$, noting that we just proved that $\varphi \dashv \vdash \mathrm{B} \varphi$, $\varphi \dashv \vdash \mathrm{B} \varphi \dashv$ BB$\varphi \dashv \widehat{\mathrm{B}} \varphi$.

Lemma 4.3 For every $\varphi, \psi \in \mathscr{L}$ Пzg, the following are theorems of $\mathrm{KD} 4^{\forall} 5 \Pi \mathrm{zg}$ :

$$
\begin{aligned}
& {[z](\varphi \rightarrow \psi) \rightarrow([z] \varphi \rightarrow[z] \psi),} \\
& z \rightarrow([z] \varphi \rightarrow \varphi), \\
& {[z] \varphi \rightarrow[z][z] \varphi,} \\
& \langle z\rangle \varphi \rightarrow[z]\langle z\rangle \varphi .
\end{aligned}
$$

Moreover, if $\vdash \mathrm{z} \rightarrow \varphi$, then $\vdash \mathrm{z} \rightarrow[\mathrm{z}] \varphi$. This means that, assuming $\mathrm{z},[\mathrm{z}]$ is an $\mathrm{S} 5$ modality.

Proof The first, third, and last formulas are easy to derive in $\mathrm{KD} 4{ }^{\forall} 5 \Pi \mathrm{zg}$. For the second, recall that by the axiom $\mathrm{z}, \vdash \mathrm{z} \leftrightarrow \forall p(\mathrm{~B} p \rightarrow p)$. Hence, assuming $\mathrm{z}$, we can deduce $\mathrm{B}(\mathbf{z} \rightarrow \varphi) \rightarrow(\mathbf{z} \rightarrow \varphi)$. But we can also derive $(\mathbf{z} \rightarrow \varphi) \rightarrow \varphi$ as we have $\mathbf{z}$ in hand. So we derive $\mathrm{B}(\mathrm{z} \rightarrow \varphi) \rightarrow \varphi$ and thus $[\mathrm{z}] \varphi \rightarrow \varphi$.

Finally, for the necessitation-like implication, suppose that $\vdash z \rightarrow \varphi$. Then by necessitation, $\mathbf{B}(\mathbf{z} \rightarrow \varphi)$ is provable, but this is just $[\mathbf{z}] \varphi$. So certainly $\mathbf{z} \rightarrow[z] \varphi$ is provable.

Moving to the $\mathscr{F} \mathscr{O L}$ zg side, what we need is a first-order logic that is weak enough to be embedded using $\mathscr{T}^{\prime}$ in $\mathrm{KD} 4^{\forall} 5 \Pi \mathrm{zg}$, yet strong enough to include all validities of the natural quotients of complete proper filter algebras. It turns out that 
this logic is the logic of the following very general class of Boolean algebras with two named elements.

Definition 4.10 A $z g$-algebra $A$ is a tuple $\left\langle A_{0}, z_{A}, g_{A}\right\rangle$ such that $A_{0}$ is a non-trivial Boolean algebra and $z_{A}, g_{A} \in A_{0}$, such that $z_{A} g_{A}$ is atomic (it is the join of atoms below it), $z_{A} \overline{g_{A}}$ is atomless (there are no atoms below it), and $g_{A} \leq z_{A}$.

Note that according to the definition, for any zg-algebra $A, g_{A}$ is precisely the join of the atoms below $z_{A}$. Hence zg-algebras can alternatively be defined as Boolean algebras with an element $z$ such that the join of the atoms below it exists and is denoted by $g$. It is not too hard to observe that all natural quotients of complete proper filter algebras are zg-algebras. On the other hand, there are certainly zg-algebras that are not isomorphic to the natural quotients of any complete proper filter algebra. An obvious way to construct such zg-algebras is to take zg-algebras whose restriction to $z$ is not complete. By our definition, this is totally admissable for being a zgalgebra: the existence of just the join of atoms below $z$ suffices. However, for any zgalgebra $A=\mathscr{B} / F_{\mathscr{B}}$ where $\mathscr{B}$ is some complete proper filter algebra, $\left.A\right|_{z_{A}}$ must be a complete Boolean algebra since first $\mathscr{B}$ is complete and second $\left.A\right|_{z_{A}}$ is isomorphic to $\left.\mathscr{B}\right|_{z_{\mathscr{B}}}$. We will show that it is not a problem that zg-algebras forms a wider class than the class of natural quotients of complete proper filter algebras. The motivation of having a wider class is that this class of zg-algebras is first-order definable, and thus we get a complete first-order logic for free. The logic is presented below, and we omit the easy soundness and completeness proof since the class of zg-algebras is obviously defined by the non-logical axioms.

Proposition 4.3 The validities of all zg-algebras in the language of $\mathscr{F} \mathscr{O L} \mathrm{zg}$ under the semantics in Definition 4.7 is axiomatized by the logic FOLzg defined by the axiom schemas below and the usual modus ponens and universalization rule. In the group of logical axioms, $\varphi, \psi$ are arbitrary formulas in $\mathscr{F} \mathscr{O L} \mathrm{zg}, t$ is an arbitrary term, and $p, q, r$ are arbitrary variables in Prop. In the second group of non-logical axioms, $p, q, r$ are three specific and distinct variables in Prop while $s, t$ still stand for arbitrary terms.

\section{Logical axioms}

All instances of propositional tautology schemas in $\mathscr{F} \mathscr{O} \mathscr{L} \mathrm{zg}$

$$
\forall p(\varphi \rightarrow \psi) \rightarrow(\forall p \varphi \rightarrow \forall p \psi)
$$

$\forall p \varphi \rightarrow \varphi[t / p]$ when $t$ is substitutable for $p$ in $\varphi$

$\varphi \rightarrow \forall p \varphi$ when $p$ is not free in $\varphi$

$(p=p) \wedge((p=q) \rightarrow(q=p))$

$((p=q) \wedge(q=r)) \rightarrow(p=r)$

$(p=q) \rightarrow((\neg p=\neg q))$

$(p=q) \rightarrow((r \wedge p=r \wedge q) \wedge(p \wedge r=q \wedge r))$ 


\section{Non-logical axioms}

$$
\begin{gathered}
\neg(\top \leqslant \perp) \\
(s=t) \text { when } s \leftrightarrow t \text { is a tautology } \\
(\mathrm{g} \leqslant \mathrm{z}) \\
\forall p(((p \leqslant \mathrm{zg}) \wedge(p \neq \perp)) \rightarrow \exists q((q \leqslant p) \wedge(q \neq \perp) \wedge \forall r((q \leqslant r) \vee(q \leqslant r)))) \\
\forall p(((p \leqslant \mathrm{z} \overline{\mathrm{g}}) \wedge(p \neq \perp)) \rightarrow \exists q((q \leqslant p) \wedge(p q \neq \perp) \wedge(p \bar{q} \neq \perp)))
\end{gathered}
$$

Note that we are not using the usual Leibniz's law in this axiomatization. Instead, we have a group of axioms saying that the equality relation is a congruence relation. The usual Leibniz's law can be derived from them together with other axioms and rules. This is mainly for the ease of showing that the translations preserve theoremhood, since $\mathrm{KD} 4^{\forall} 5 \Pi \mathrm{zg}$ does not have Leibniz's law as one of its axioms.

In the non-logical axioms above and also for the rest of the paper, we use the following abbreviations in $\mathscr{F} \mathscr{O} \mathscr{L} \mathrm{zg}:(s \leqslant t):=((s \rightarrow t)=\top)$ and $(s \neq t):=$ $\neg(s=t)$. Intuitively the abbreviation $(s \leqslant t)$ says that $s$ is below $t$ in the Boolean lattice order. Then the two axioms intuitively say that $\mathrm{zg}$ is atomic and $\mathrm{z} \overline{\mathrm{g}}$ is atomless respectively. Obviously then the non-logical axioms define zg-algebras.

That FOLzg is weak enough to be embedded into $\mathrm{KD} 4^{\forall} 5 \Pi \mathrm{zg}$ is shown by the following three lemmas.

Lemma 4.4 For any $\varphi \in \mathscr{L}$ B Пzg, $\varphi \leftrightarrow \mathscr{T}^{\prime}(\mathscr{T}(\varphi))$ is provable in $\mathrm{KD} 4{ }^{\forall} 5 \Pi z g$. For any $\varphi \in \mathscr{F} \mathscr{O L} \mathbf{z g}, \varphi \leftrightarrow \mathscr{T}\left(\mathscr{T}^{\prime}(\varphi)\right)$ is provable in FOLzg

Proof $\mathscr{T}^{\prime}(\mathscr{T}(\varphi))$ turns every $\mathrm{B} \beta$ in $\varphi$ first to $\beta=\top$ and then to $\mathrm{B}(\beta \leftrightarrow \top)$. But $\mathrm{B} \beta \leftrightarrow \mathrm{B}(\beta \leftrightarrow \top)$ is in $\mathrm{KD} 4^{\forall} 5 \Pi z g$. Similarly, $\mathscr{T}\left(\mathscr{T}^{\prime}(\varphi)\right)$ turns the $s=t$ in $\varphi$ first to $\mathrm{B}(s \leftrightarrow t)$ and then to $((s \leftrightarrow t)=\top)$. But $(s=t) \leftrightarrow((s \leftrightarrow t)=\top)$ is in FOLzg.

Lemma 4.5 For any axiom $\varphi$ in Proposition 4.3 defining $\mathscr{F} \mathscr{O L} \mathrm{zg}, \mathscr{T}^{\prime}(\varphi)$ is provable in $\mathrm{KD} 4{ }^{\forall} 5$ П $\mathrm{zg}$.

Proof The translations of the logical axioms are easily provable in $\mathrm{KD} 4^{\forall} 5 \Pi \mathrm{zg}$ since it is a normal $\Pi$-logic and, in particular it can do Boolean reasoning inside B. For the rest, the only non-trivial axioms to be dealt with are Eqs. 6 and 7. To derive the translation of Eq. 6 in $\mathrm{KD} 4^{\forall} 5 \Pi z g$, we now work in $\mathscr{L} \Pi z \mathrm{zg}$. Let us first assume $p z$ in system. Then we have $p \wedge \forall p(\mathrm{~B} p \rightarrow p) \wedge \exists p(p \wedge a t(p))$. Instantiating $\exists p(p \wedge a t(p))$ using $x$, we have $x \wedge a t(x)$ that just abbreviates

$$
x \wedge\langle\mathrm{z}\rangle x \wedge \forall r([\mathrm{z}](x \rightarrow r) \vee[\mathrm{z}](x \rightarrow \neg r)) .
$$

Instantiating $\forall r([\mathrm{z}](x \rightarrow r) \vee[\mathrm{z}](x \rightarrow \neg r))$ using $p$, we have $[\mathrm{z}](x \rightarrow p) \vee[\mathrm{z}](x \rightarrow$ $\neg p)$. But the latter disjunct leads to contradiction since we have assumed $\mathrm{z}$, which, according to Lemma 4.3, allows us to remove [z] and obtain $x \rightarrow \neg p$, contradicting 
the previously assumed $p z g$ and $x \wedge a t(x)$. Hence, we reject the second disjunct and derive $[z](x \rightarrow p)$. Summing everything, we now have:

$$
[\mathrm{z}](x \rightarrow p) \wedge\langle\mathrm{z}\rangle x \wedge \forall r([\mathrm{z}](x \rightarrow r) \vee[\mathrm{z}](x \rightarrow \neg r)) .
$$

Writing this without any abbreviation and using $\varphi_{1} \rightarrow\left(\varphi_{2} \rightarrow \varphi_{3}\right)$ being provably equivalent to $\left(\varphi_{1} \wedge \varphi_{2}\right) \rightarrow \varphi_{3}$, we then have

$$
\mathrm{B}((\mathrm{z} \wedge x) \rightarrow p) \wedge \widehat{\mathrm{B}}(\mathrm{z} \wedge x) \wedge \forall r(\mathrm{~B}((\mathrm{z} \wedge x) \rightarrow r) \vee \mathrm{B}((\mathrm{z} \wedge x) \rightarrow \neg r)) .
$$

Then we can existentially quantify back to obtain

$$
\varphi(p):=\exists q(\mathrm{~B}(q \rightarrow p) \wedge \widehat{\mathrm{B}} q \wedge \forall r(\mathrm{~B}(q \rightarrow r) \vee \mathrm{B}(q \rightarrow \neg r))),
$$

as $\mathrm{z} \wedge x$ is a witness. The above process shows that $\vdash p z g \rightarrow \varphi(p)$.

Now assume $\mathrm{B}(p \rightarrow \mathrm{zg}) \wedge \widehat{\mathrm{B}} p$ in system. Then clearly we can deduce $\widehat{\mathrm{B}}(p \mathrm{zg})$. Since we have just shown that $\vdash p$ zg $\rightarrow \varphi(p)$, we also obtain $\mathrm{B}(p z g \rightarrow \varphi(p))$. Thus $\widehat{\mathrm{B}}(p z g) \rightarrow \widehat{\mathrm{B}}(\varphi(p))$ is provable. But $\varphi(p)$ is fully modalized. So by Lemma 4.2, $\widehat{\mathrm{B}}(\varphi(p)) \dashv \vdash \varphi(p)$. This means that $(\mathrm{B}(p \rightarrow \mathrm{zg}) \wedge \widehat{\mathrm{B}} p) \rightarrow \varphi(p)$ is provable, and hence, after universalization, $\vdash \forall p((\mathrm{~B}(p \rightarrow \mathrm{zg}) \wedge \widehat{\mathrm{B}} p) \rightarrow \varphi(p))$.

Note that for any $s, t \in \mathscr{L}$ Boolzg, $\mathscr{T}(\mathrm{B}(s \rightarrow t))=(s \leqslant t)$ and $\mathscr{T}(\widehat{\mathrm{B}} s)=(\neg s \neq$ $\perp)$. The latter is easily seen to be provably equivalent to $(s \neq \top)$ in FOLzg. Thus, $\mathscr{T}(\forall p((\mathrm{~B}(p \rightarrow \mathrm{zg}) \wedge \widehat{\mathrm{B}} p) \rightarrow \varphi(p)))$ is obviously provably equivalent to Eq. 6 in FOLzg. By Lemma 4.4, we are done in this case.

The translation of Eq. 7 can be derived in $\mathrm{KD} 4{ }^{\forall} 5 \Pi \mathrm{zg}$ similarly. The key again is that once we assume $z,[z]$ is an S5 modality.

Lemma 4.6 For any $\varphi \in$ FOLzg, $\mathscr{T}^{\prime}(\varphi) \in \mathrm{KD} 4^{\forall} 5 \Pi z g$.

Proof We show that for any derivation $\left\langle\varphi_{1}, \varphi_{2}, \cdots, \varphi_{n}\right\rangle$ of FOLzg, $\mathscr{T}^{\prime}\left(\varphi_{i}\right)$ is a theorem of $\mathrm{KD} 4^{\forall} 5 \Pi \mathrm{zg}$ for all $i$ from 1 to $n$ by induction. For any $i$, if $\varphi_{i}$ is an axiom in FOLzg, then by the previous lemma, $\mathscr{T}^{\prime}(\varphi) \in \mathrm{KD} 4^{\forall} 5 \Pi \mathrm{zg}$. If $\varphi_{i}$ is obtained by either modus ponens or universalization, notice that the same rule applies to the formulas after translation as the translation does not change the sentential form or the variables used. So $\mathscr{T}^{\prime}\left(\varphi_{i}\right)$ can also be obtained from the rules.

Now we can show the completeness of $\mathrm{KD} 4{ }^{\forall} 5 \Pi \mathrm{zg}$ from the following two lemmas.

Lemma 4.7 Any sentence in $\mathscr{L}$ Пzg is equivalent in $\mathrm{KD} 4{ }^{\forall} 5 \Pi \mathrm{zg}$ to a sentence in the following form:

$$
(\overline{\mathrm{z}} \wedge \alpha) \vee(\mathrm{zg} \wedge \beta) \vee(\mathrm{z} \overline{\mathrm{g}} \wedge \gamma),
$$

where $\alpha, \beta, \gamma$ are all sentences in $\mathscr{L} \mathrm{B} \Pi z \mathrm{z}$.

Lemma 4.8 For any zg-algebra A, there is a complete proper filter algebra $\mathscr{B}$ such that $\mathscr{B} / F_{\mathscr{B}}$ is elementarily equivalent to $A$ (satisfying the same formulas in $\mathscr{F} \mathscr{O L} \mathbf{z g})$.

Moreover, $\overline{z_{\mathscr{B}}}$ is not $\perp_{\mathscr{B}}, z_{\mathscr{B}} g_{\mathscr{B}}$ is $\perp_{\mathscr{B}}$ if and only if $z_{A} g_{A}$ is $\perp_{A}$, and $z_{\mathscr{B}} \overline{g_{\mathscr{B}}}$ is $\perp_{\mathscr{B}}$ if and only if $z_{A} \overline{g_{A}}$ is $\perp_{A}$. 
Theorem 4.2 Any sentence in $\mathscr{L} \Pi z g$ that is valid on all complete proper filter algebras is also in $\mathrm{KD} 4{ }^{\forall} 5$ Пg.

Proof By Lemma 4.7, we can assume that we are dealing with a sentence $\varphi=(\overline{\mathbf{z}} \wedge$ $\alpha) \vee(\mathrm{zg} \wedge \beta) \vee(\mathrm{z} \overline{\mathrm{g}} \wedge \gamma)$ where $\alpha, \beta, \gamma$ are all sentences in $\mathscr{L} \mathrm{B} \Pi z \mathrm{~g}$. To proceed, assume that $\varphi$ is valid on all complete proper filter algebras.

By Lemma 4.1, for any complete proper filter algebra $\mathscr{B}$ and any valuation $\theta$ on $\mathscr{B}, \tilde{\theta}(\alpha), \tilde{\theta}(\beta), \tilde{\theta}(\gamma)$ are either T or $\perp$. Also, $\bar{z}, z g$, and $z \bar{g}$ disjointly decompose $\mathscr{B}$. This means that once $\tilde{\theta}(\overline{\mathbf{z}}) \neq \perp, \tilde{\theta}(\alpha)$ must be T since otherwise $\tilde{\theta}(\varphi)$ will lose a non-trivial $\tilde{\theta}(\bar{z})$ and be strictly below $\mathrm{T}$. Similarly, $\tilde{\theta}(\mathrm{zg}) \neq \perp$ implies $\tilde{\theta}(\beta)=\mathrm{T}$, and $\tilde{\theta}(\mathrm{z} \overline{\mathrm{g}}) \neq \perp$ implies $\tilde{\theta}(\gamma)=\mathrm{T}$.

Focus on $\alpha$ first. Now we know that $\alpha$ is valid on all complete proper filter algebra $\mathscr{B}$ where $\overline{z_{\mathscr{B}}} \neq \perp$ since $\overline{\mathrm{z}}$ always evaluates to $\overline{z_{\mathscr{B}}}$. Then for any zg-algebra $A, A \vDash$ $\mathscr{T}(\alpha)$ since by Lemma 4.8 , for any zg-algebra $A$ there is a complete proper filter algebra $\mathscr{B}$ with $\overline{z_{\mathscr{B}}} \neq \perp$ such that $\mathscr{B} / F_{\mathscr{B}}$ is elementarily equivalent to $A$, and by Lemma 4.1, $\mathscr{B} / F_{\mathscr{B}} \vDash \mathscr{T}(\alpha)$ iff $\mathscr{B} \vDash \alpha$. Now that $\mathscr{T}(\alpha)$ is valid on all zg-algebras, by Proposition 4.3, $\mathscr{T}(\alpha) \in \mathscr{F} \mathscr{O} \mathscr{L}$ zg. By Lemma 4.6 and 4.4, then, $\vdash \alpha$, and thus $\vdash z \rightarrow \alpha$.

The method applies to the cases with $\beta$ and $\gamma$ too. Take $\beta$ as an example. Now $\beta$ must be valid on all complete proper filter algebra $\mathscr{B}$ where $z_{\mathscr{B}} g_{\mathscr{B}}$ is non-trivial. If $\mathscr{T}(\beta)$ is refutable by some zg-algebra $A$ with $z_{A} g_{A}$ non-trivial, then $\beta$ will also be refutable by some complete proper filter algebra $\mathscr{B}$ with $z_{\mathscr{B}} g_{\mathscr{B}}$ non-trivial, using lemma 4.8 and 4.1. This means that $\mathscr{T}(\beta)$ is valid on any zg-algebra $A$ with $z_{A} g_{A}$ non-trivial. In other words, $\neg(\mathrm{zg}=\perp) \rightarrow \mathscr{T}(\beta)$ is valid and hence provable in $\mathscr{F} \mathscr{O L}$ zg. Translating back to $\mathscr{L} \Pi z \mathrm{zg}, \vdash \widehat{\mathrm{B}}(\mathrm{zg}) \rightarrow \beta$. But $\vdash \mathrm{zg} \rightarrow \widehat{\mathrm{B}}(\mathrm{zg})$ since $\mathrm{z} \dashv \vdash \forall p(p \rightarrow \widehat{\mathrm{B}} p)$, and then we can instantiate this with zg. So $\vdash \mathrm{zg} \rightarrow \beta$. In the same fashion, it can be shown that $\vdash z \bar{g} \rightarrow \gamma$.

Taking stock, we have shown that $\vdash(\overline{\mathrm{z}} \rightarrow \alpha), \vdash(\mathrm{zg} \rightarrow \beta)$, and $\vdash(\mathrm{z} \overline{\mathrm{g}} \rightarrow \gamma)$. But obviously $\vdash \bar{z} \vee z g \vee z \bar{g}$ since trivially $\vdash \mathrm{g} \rightarrow \mathrm{z}$. With some basic Boolean manipulation, $\vdash(\overline{\mathrm{z}} \wedge \alpha) \vee(\mathrm{zg} \wedge \beta) \vee(\mathrm{z} \overline{\mathrm{g}} \wedge \gamma)$.

Theorem 4.1 follows since $\mathrm{KD} 4{ }^{\forall} 5 \Pi \mathrm{zg}$ is a conservative extension of $\mathrm{KD} 4^{\forall} 5 \Pi$, and a formula is valid if and only if its universal closure, which is a sentence, is valid.

\subsection{Syntactical Reduction}

In this section, we prove Lemma 4.7. The main idea is relativizing formulas by $\mathrm{zg}, \mathrm{z} \overline{\mathrm{g}}$, and $\bar{z}$. We also use ideas from the quantifier elimination for $\mathrm{S} 5 \Pi$. See the appendix of [40] and the original [15] for more about the quantifier elimination for S5 П.

An important addition to the $55 \Pi$ case is the following lemma, where the intuition is that if $\neg \widehat{\mathrm{B}} b$ is true, then $b$ is unimportant and does not affect the semantic value of $\varphi(p)$ where $\varphi \in \mathscr{L} \mathrm{B} \Pi z \mathrm{~g}$, when we replace $p$ by either $p \vee b$ or $p \wedge \neg b$.

Lemma 4.9 For any formula $\varphi(p) \in \mathscr{L} \mathrm{B} \Pi z g$ where $p$ is free and any propositional variable $b$ not occuring in $\varphi$, the following are provable in $\mathrm{KD} 4{ }^{\forall} 5$ П zg:

$$
\mathrm{B} \neg b \rightarrow(\varphi(p) \leftrightarrow \varphi(p \vee b)), \quad \mathrm{B} \neg b \rightarrow(\varphi(p) \leftrightarrow \varphi(p \wedge \neg b)) .
$$


Proof We only show the $p \vee b$ case here. The other case can be shown similarly. First, a simple induction shows that for any Boolean formula $\beta(p),(\beta(p) \vee b) \dashv \vdash$ $(\beta(p \vee b) \vee b)$. The only non-trivial case is negation. If $(\beta(p) \vee b) \dashv(\beta(p \vee b) \vee b)$, then $\neg(\beta(p) \vee b) \dashv \vdash \neg(\beta(p \vee b) \vee b)$. Pushing $\neg$ inside, $(\neg \beta(p) \wedge \neg b) \dashv \vdash(\neg \beta(p \vee$ $b) \wedge \neg b)$. Joining a $b$ on both side and performing some Boolean manipulation, we see that $(\neg \beta(p) \vee b) \dashv \vdash(\neg \beta(p \vee b) \vee b)$.

Using the normality of $\mathrm{B}$, it is not hard to see that $\vdash \mathrm{B} \neg b \rightarrow(\mathrm{B} \varphi \leftrightarrow \mathrm{B}(\varphi \vee b))$ : assuming $\mathrm{B} \neg b, \mathrm{~B}(\varphi \vee b)$ implies $\mathrm{B}((\varphi \vee b) \wedge \neg b)$, which then implies $\mathrm{B}(\varphi \wedge \neg b)$ and hence also $\mathrm{B} \varphi$. The other direction is trivial. Applying this to the case of $\varphi$ being $\beta(p)$, we see then that $\vdash \mathrm{B} \neg b \rightarrow(\mathrm{B} \beta(p) \leftrightarrow \mathrm{B}(\beta(p) \vee b))$. Using the claim we proved in the last paragraph, $\vdash \mathrm{B} \neg b \rightarrow(\mathrm{B} \beta(p) \leftrightarrow \mathrm{B} \beta(p \vee b))$. This forms the basis of a trivial induction on the formulas in $\mathscr{L} \mathrm{B} \Pi z \mathrm{zg}$.

Since our strategy is to relativize by $z g, z \bar{g}$, and $\bar{z}$, we first introduce the necessary definitions and lemmas required for separating the $z g$ and $z \bar{g}$ part. Then we move to the necessary preparation for separating the $\bar{z}$ part. Then we show a simple lemma on when we can push $\exists p$ over conjunctions. After that, we combine everything together.

For the $z g$ and $z \bar{g}$ part, we need the following abbreviations:

$$
M_{i} \varphi:=\exists p_{1} \cdots p_{i}\left(\bigwedge_{1 \leq i<j \leq n}[\mathrm{z}]\left(p_{i} \rightarrow \neg p_{j}\right) \wedge \bigwedge_{1 \leq i \leq n} \operatorname{at}\left(p_{i}\right) \wedge \bigwedge_{1 \leq i \leq n}[\mathrm{z}]\left(p_{i} \rightarrow \varphi\right)\right)
$$

(for $i \geq 1$ ),

$M_{0} \varphi:=\top, \quad Q_{i} \varphi:=M_{i} \varphi \wedge \neg M_{i+1} \varphi($ for $i \in \mathbb{N}), \quad N \varphi:=\langle\mathrm{z}\rangle(\bar{g} \wedge \varphi)$.

As usual, the auxiliary variables are chosen to be distinct and unused in $\varphi$. Here $M_{i}, Q_{i}$, and $N$ come from the quantifier elimination proof of [15], which requires the modality to be S5. But by Lemma 4.3, the modality [z] used here is really S5 if $\mathrm{z}$ is also present. Even if $\mathrm{z}$ is not assumed, $\mathrm{KD} 4{ }^{\forall} 5 \Pi \mathrm{zg}$ still proves many intuitive principles. We summarize the results in the following lemma.

Lemma 4.10 $\mathrm{KD} 4{ }^{\forall} 5 \Pi \mathrm{zg}$ proves all instances of the following schemas. In the last group, $q$ is required to be not free in $\varphi$ and $\pm q$ can be either $q$ or $\neg q$. Moreover, when $\pm q$ is taken to be $q, m$ in the first four formulas is not 0 , and when $\pm q$ is taken to be $\neg q, n$ is not 0 .

$$
\langle\mathrm{z}\rangle(\mathrm{g} \wedge \varphi) \leftrightarrow M_{1} \varphi \quad M_{i} \varphi \leftrightarrow M_{i}(\mathrm{~g} \wedge \varphi) \quad Q_{i} \varphi \leftrightarrow Q_{i}(\mathrm{~g} \wedge \varphi)
$$

$\begin{array}{llll}\exists q\left[Q_{m}(\varphi \wedge q) \wedge Q_{n}(\varphi \wedge \bar{q})\right] & \leftrightarrow Q_{m+n} \varphi & \exists q[N(\varphi \wedge q) \wedge N(\varphi \wedge \bar{q})] & \leftrightarrow N \varphi \\ \exists q\left[Q_{m}(\varphi \wedge q) \wedge M_{n}(\varphi \wedge \bar{q})\right] & \leftrightarrow M_{m+n} \varphi & \exists q[N(\varphi \wedge q) \wedge \neg N(\varphi \wedge \bar{q})] & \leftrightarrow N \varphi \\ \exists q\left[M_{m}(\varphi \wedge q) \wedge Q_{n}(\varphi \wedge \bar{q})\right] & \leftrightarrow M_{m+n} \varphi & \exists q[\neg N(\varphi \wedge q) \wedge N(\varphi \wedge \bar{q})] & \leftrightarrow N \varphi \\ \exists q\left[M_{m}(\varphi \wedge q) \wedge M_{n}(\varphi \wedge \bar{q})\right] & \leftrightarrow M_{m+n} \varphi & \exists q[\neg N(\varphi \wedge q) \wedge \neg N(\varphi \wedge \bar{q})] & \leftrightarrow \neg N \varphi \\ \bigvee_{i=0}^{n}\left(M_{i}(\varphi \wedge \psi) \wedge M_{n-i}(\varphi \wedge \bar{\psi})\right) & \leftrightarrow M_{n} \varphi & ((N(\varphi \wedge \psi) \vee(N(\varphi \wedge \bar{\psi}))) & \leftrightarrow N \varphi \\ \bigvee_{i=0}^{n}\left(Q_{i}(\varphi \wedge \psi) \wedge Q_{n-i}(\varphi \wedge \bar{\psi})\right) & \leftrightarrow Q_{n} \varphi & ((\neg N(\varphi \wedge \psi) \wedge \neg(N(\varphi \wedge \bar{\psi}))) \leftrightarrow \neg N \varphi\end{array}$




$$
\begin{aligned}
& \exists q\left[\mathrm{zg} \wedge \varphi \wedge \pm q \wedge Q_{m}(\varphi \wedge q) \wedge Q_{n}(\varphi \wedge \bar{q})\right] \leftrightarrow\left(\mathrm{zg} \wedge \varphi \wedge Q_{m+n} \varphi\right) \\
& \exists q\left[\mathrm{zg} \wedge \varphi \wedge \pm q \wedge M_{m}(\varphi \wedge q) \wedge Q_{n}(\varphi \wedge \bar{q})\right] \leftrightarrow\left(\mathrm{zg} \wedge \varphi \wedge M_{m+n} \varphi\right) \\
& \exists q\left[\mathrm{zg} \wedge \varphi \wedge \pm q \wedge Q_{m}(\varphi \wedge q) \wedge M_{n}(\varphi \wedge \bar{q})\right] \leftrightarrow\left(\mathrm{zg} \wedge \varphi \wedge M_{m+n} \varphi\right) \\
& \exists q\left[\mathrm{zg} \wedge \varphi \wedge \pm q \wedge M_{m}(\varphi \wedge q) \wedge M_{n}(\varphi \wedge \bar{q})\right] \leftrightarrow\left(\mathrm{zg} \wedge \varphi \wedge M_{m+n} \varphi\right) \\
& \exists q[\mathrm{z} \overline{\mathrm{g}} \wedge \varphi \wedge \pm q \wedge N(\varphi \wedge q) \wedge N(\varphi \wedge \bar{q})] \leftrightarrow(\mathrm{z} \overline{\mathrm{g}} \wedge \varphi \wedge N \varphi) \\
& \exists q[\mathrm{z} \overline{\mathrm{g}} \wedge \varphi \wedge \pm q \wedge \neg N(\varphi \wedge q) \wedge N(\varphi \wedge \bar{q})] \leftrightarrow(\mathrm{z} \overline{\mathrm{g}} \wedge \varphi \wedge N \varphi) \\
& \exists q[\mathrm{z} \overline{\mathrm{g}} \wedge \varphi \wedge \pm q \wedge N(\varphi \wedge q) \wedge \neg N(\varphi \wedge \bar{q})] \leftrightarrow(\mathrm{z} \overline{\mathrm{g}} \wedge \varphi \wedge N \varphi) \\
& \exists q[\mathrm{z} \overline{\mathrm{g}} \wedge \varphi \wedge \pm q \wedge \neg N(\varphi \wedge q) \wedge \neg N(\varphi \wedge \bar{q})] \leftrightarrow(\mathrm{z} \overline{\mathrm{g}} \wedge \varphi \wedge \neg N \varphi)
\end{aligned}
$$

Proof Syntactical proofs of them are not interesting, and here we only briefly explain why they are valid, from which syntactical proofs can be extracted straightforwardly. Using Proposition 4.2, $M_{i} \varphi$ says that $\mathrm{z} \wedge \varphi$ contains at least $i$ atoms. More precisely, for any complete proper filter algebra $\mathscr{B}$ and valuation $\theta$ on $\mathscr{B}, \tilde{\theta}\left(M_{i} \varphi\right)=\top$ if and only if $z_{\mathscr{B}} \wedge \tilde{\theta}(\varphi)$ contains at least $i$ atoms, and otherwise $\tilde{\theta}\left(M_{i} \varphi\right)=\perp$. Similarly, $Q_{i} \varphi$ says that $\mathrm{z} \wedge \varphi$ contains exactly $i$ atoms, and $N \varphi$ says that $\mathrm{z} \wedge \varphi$ contains an atomless part.

Note that $\tilde{\theta}(\mathrm{g})=g_{\mathscr{B}}$ is the join of all atoms under $z_{\mathscr{B}}$ and hence atomic. So if $g_{\mathscr{B}} \wedge z_{\mathscr{B}} \wedge \tilde{\theta}(\varphi)$ is non-trivial, then $z_{\mathscr{B}} \wedge \tilde{\theta}(\varphi)$ must contain an atom, and the numbers of atoms below $z_{\mathscr{B}} \wedge \tilde{\theta}(\varphi)$ and $g_{\mathscr{B}} \wedge z_{\mathscr{B}} \wedge \tilde{\theta}(\varphi)$ respectively are the same. These two observations show the validity of the first group of three formulas.

The left six formulas in the second group are simply counting principles, and the right six formulas state obvious properties of atomless elements. Hence they are all valid. Note that they only consider the situation under $z$.

For the last group, note that by Boolean reasoning, $(\mathrm{z} \wedge \alpha) \leftrightarrow(\mathrm{z} \wedge \beta) \dashv \vdash \mathrm{z} \rightarrow$ $(\alpha \leftrightarrow \beta)$. By Proposition 4.3, to prove the last group of formulas in $\mathrm{KD} 4{ }^{\forall} 5 \Pi \mathrm{zg}$, we only need to translate their proofs in $\mathrm{S} 5 \Pi$ to proofs in $\mathrm{KD} 4{ }^{\forall} 5 \Pi \mathrm{zg}$ by replacing the S5 modality $\square$ by [z].

For the $\bar{z}$ part, the only extra definition we need is the following.

Definition 4.11 Define the following abbreviations:

$$
\langle\overline{\mathrm{z}}\rangle \varphi:=\widehat{\mathrm{B}}(\overline{\mathrm{Z}} \wedge \varphi), \quad[\overline{\mathrm{z}}] \varphi:=\mathrm{B}(\overline{\mathrm{z}} \rightarrow \varphi) .
$$

Then define the following restricted version of $\mathscr{L}$ BПzg:

$$
\mathscr{L}[\overline{\mathbf{z}}] \Pi: \varphi::=[\overline{\mathrm{z}}] t|\neg \varphi|(\varphi \wedge \varphi) \mid \forall p \varphi
$$

where $t \in \mathscr{L}$ Bool, $p \in$ Prop.

Now we introduce the concept of a propositional variable being restricted. This helps us to distribute existential quantifiers over conjunctions in certain cases.

Definition 4.12 We say that $p$ is restricted by a formula $\mu$ in $\varphi$ just in case $\mu$ is substitutable for $p$ in $\varphi$ and $\vdash \forall p(\varphi(p) \leftrightarrow \varphi(p \wedge \mu))$. 
Lemma 4.11 $\exists p(\varphi \wedge \psi)$ is provably equivalent to $\exists p \varphi \wedge \exists p \psi$, if there are formulas $\mu, v$, such that

- $\quad$ p in $\varphi$ is restricted by $\mu, p$ in $\psi$ is restricted by $\nu$, and

$-\quad \neg(\mu \wedge v)$ is provable.

Proof One direction of the equivalence is trivial. For the other, the strategy is relativization. Suppose $\exists p \varphi(p) \wedge \exists p \psi(p)$ in system. Then we have $\varphi\left(p_{1}\right)$ and $\psi\left(p_{2}\right)$. By the assumption that $p$ in $\varphi$ is restricted by $\mu$ and that $p$ in $\psi$ is restricted by $\nu$, we can derive $\varphi\left(p_{1} \wedge \mu\right)$ and $\psi\left(p_{2} \wedge \nu\right)$. Now we see that $\neg(\mu \wedge \nu)$ is provable. So, using Boolean reasoning and letting $\chi=\left(p_{1} \wedge \mu\right) \vee\left(p_{2} \wedge v\right), \chi \wedge \mu \dashv \vdash p_{1} \wedge \mu$ and $\chi \wedge v \dashv \vdash p_{2} \wedge v$. Hence we now have a chain of provable equivalence: $\varphi(\chi) \dashv \varphi(\chi \wedge \mu) \dashv \vdash \varphi\left(p_{1} \wedge \mu\right) \dashv \vdash \varphi\left(p_{1}\right)$. Similarly $\psi(\chi) \dashv \psi \psi\left(p_{2}\right)$. Thus $\chi$ witness $\exists p(\varphi(p) \wedge \psi(p))$.

Now we start to combine everything together. A few extra notations are used. We fix an enumeration $\left\langle p_{i}\right\rangle_{i<\mid \text { Prop } \mid}$ of Prop and write $\mathbf{p}$ or in general use vector notation for a finite subset of Prop. Then for $\mathbf{p}=\left\{p_{i_{1}}, p_{i_{2}}, \ldots, p_{i_{n}}\right\}$ with $i_{1}<i_{2}<\cdots<i_{n}$, $2^{\mathbf{p}}$ is the set of formulas in the form of $\pm p_{i_{1}} \wedge \pm p_{i_{2}} \wedge \cdots \wedge \pm p_{i_{n}}$. We call elements in $2^{\mathbf{p}}$ cells.

And as usual, a conjunction of no formulas is $\top$, and a disjunction of no formulas is $\perp$.

Definition 4.13 A state description over $\mathbf{p}$ with degree $l \in \mathbb{N}$ is a conjunction of the following components:

- (choice of zg) one of $\bar{z}, z g, z \bar{g}$,

- (propositional truth) one cell $c \in 2^{\mathbf{p}}$,

- ( $\bar{z}$ part) one $\mathscr{L}[\bar{z}] \Pi$ formula $\delta$ whose free variables are all in $\mathbf{p}$,

- (zg part) for each cell $c \in 2^{\mathbf{p}}$, a formula $M_{l} c$ or $Q_{i} c$ for some $0 \leq i<l$,

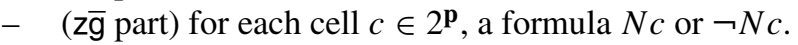

We call the first two parts the propositional part and the rest the modal part of a state description. A partial state description over $\mathbf{p}$ of degree $l$ is a formula missing one or more components above. If the only missing part is the propositional part, we also call it a modal state description.

Lemma 4.12 Every free variable in a $\overline{\mathrm{z}}$ (respectively $\mathrm{zg}, \mathrm{z} \overline{\mathrm{g}}$ ) part is restricted by $\overline{\mathrm{z}}$ (respectively $\mathrm{zg}, \mathrm{z} \overline{\mathrm{g}}$ ).

Proof For the $\bar{z}$ part, note that in any formula $\varphi \in \mathscr{L}[\bar{z}] \Pi$, every free variable appears in a Boolean term which is then in a conjunction with $\bar{z}$. We can distribute this $\bar{z}$ into the Boolean term, assuming that Boolean term is already in negation normal form. Then every free variable appears either in the form of $\bar{z} \wedge p$ or $\bar{z} \wedge \neg p$. But $\overline{\mathbf{z}} \wedge p \dashv \vdash \overline{\mathbf{z}} \wedge(p \wedge \overline{\mathbf{z}})$ and $\overline{\mathbf{z}} \wedge \neg p \dashv \vdash \overline{\mathrm{z}} \wedge \neg(p \wedge \overline{\mathbf{z}})$.

For the zg part, take $M_{i} c$ for some $c \in 2^{\mathbf{p}}$ and $p \in \mathbf{p}$ for example. First note that by definition of $M_{i}, c$ in $M_{i} c$ appears in a Boolean term directly following [z]. So 
using a similar proof as in the previous case, $p$ in $M_{i} c$ is restricted to $\mathrm{z}$. Then note that in Lemma $4.10, M_{i} c$ is provably equivalent to $M_{i}(\mathrm{~g} \wedge c)$. So obviously $p$ in $M_{i} c$ is restricted to $\mathrm{g}$ as well. Finally, it is not hard to see that in general if $p$ in $\varphi(p)$ is restricted to both $\mu$ and $v$, then it is also restricted to $\mu \wedge v$. Indeed, $\varphi(p)$ will first be equivalent to $\varphi(p \wedge \mu)$ and then to $\varphi((p \wedge \mu) \wedge v)$, but this is equivalent to $\varphi(p \wedge(\mu \wedge v))$. Thus $p$ in $M_{i} c$ is restricted to zg.

The case for the $z \bar{g}$ part is similar.

Lemma 4.13 For every partial state description $\varphi$ over $\mathbf{p}$ with degree $l$, and for every finite set of variables $\mathbf{p}^{\prime} \supseteq \mathbf{p}$ and every natural number $l^{\prime} \geq l, \varphi$ is provably equivalent in $\mathrm{KD} 4{ }^{\forall} 5$ Пzg to a disjunction of state descriptions over $\mathbf{p}^{\prime}$ with degree $l^{\prime}$.

Proof Let $\varphi, \mathbf{p}, l, \mathbf{p}^{\prime}$, and $l^{\prime}$ be arbitrarily given as above. Without loss of generality, we assume that $\mathbf{p}^{\prime}=\mathbf{p} \cup\left\{p^{\prime}\right\}$ since we can repeat the following process many times if necessary. Now, let $\psi$ be the conjunction of the following $\psi_{1}, \psi_{2}, \psi_{3}, \psi_{4}$, and $\psi_{5}$ :

- If $\varphi$ has a choice of zg, let $\psi_{1}$ be this choice. Otherwise let $\psi_{1}$ be $\bar{z} \vee z g \vee z \bar{g}$.

- If $\varphi$ has a propositional truth $c \in 2^{\mathbf{p}}$, let $\psi_{2}$ be $\left(c \wedge p^{\prime}\right) \vee\left(c \wedge \overline{p^{\prime}}\right)$. Otherwise, let $\psi_{2}$ be $\bigvee 2^{\mathbf{p}^{\prime}}$.

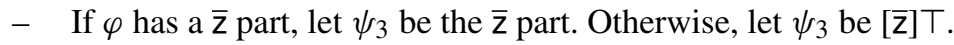

- If $\varphi$ has no zg part, let $\psi_{4}$ be the disjunctive normal form of $\bigwedge_{c \in 2^{p^{\prime}}}\left(Q_{0} c \vee Q_{1} c \vee\right.$ $\cdots \vee Q_{l^{\prime}-1} c \vee M_{l^{\prime}} c$ ) with $Q_{i} c$ and $M_{l^{\prime}} c$ seen as atomic formulas.

Otherwise, say $\varphi$ has a zg part $\delta_{\mathrm{zg}}=\bigwedge_{c \in 2^{\mathrm{p}}} X_{c} c$ with $X_{c}$ being either $Q_{i}$ for some $i<l$ or $M_{l}$. If $\vec{p}^{\prime}$ is just $\vec{p}$ (that is, $p^{\prime} \in \vec{p}$ ), let $\psi_{4}$ be $\delta_{\mathrm{zg}}$. If $p^{\prime} \notin \mathbf{p}$, construct $\psi_{4}$ by first replacing each $M_{l} c$ in $\delta_{\mathrm{zg}}$ with $\bigvee_{i=0}^{l}\left(M_{i}\left(c p^{\prime}\right) \wedge M_{l-i}\left(c \overline{p^{\prime}}\right)\right)$ and each $Q_{j} c$ with $\bigvee_{i=0}^{j}\left(Q_{i}\left(c p^{\prime}\right) \wedge Q_{j-i}\left(c \overline{p^{\prime}}\right)\right)$. Then replace each $M_{i} c$ with $c$ now being in $2^{\mathbf{p}^{\prime}}$ and with $i<l^{\prime}$ by $\left(Q_{i} c \vee Q_{i+1} c \vee \cdots \vee Q_{l^{\prime}-1} c \vee M_{l^{\prime}} c\right)$. Finally, take its disjunctive normal form with $Q_{i} c$ and $M_{l^{\prime}} c$ for all $c \in 2^{\mathbf{p}^{\prime}}$ seen as atomic formulas.

- If $\varphi$ has no z $\bar{g}$ part, let $\psi_{5}$ be the disjunctive normal form of $\bigwedge_{c \in 2^{p^{\prime}}}(N c \vee \neg N c)$ with $N c$ and $\neg N c$ seen as atomic formulas.

Otherwise, say $\varphi$ has a z $\bar{g}$ part $\delta_{\mathrm{zg}}=\bigwedge_{c \in 2 \mathrm{p}} X_{c} c$ where $X_{c}$ is either $N$ or $\neg N$. If $\mathbf{p}=\mathbf{p}^{\prime}$ (that is, $p^{\prime} \in \mathbf{p}$ ), let $\psi_{5}$ be $\delta_{z \bar{g}}$. If not, let $\psi_{5}$ be constructed by first replacing each $N c$ in $\delta_{\mathrm{z} \overline{\mathrm{g}}}$ by $\left(N\left(c p^{\prime}\right) \wedge N\left(c \overline{p^{\prime}}\right)\right) \vee\left(N\left(c p^{\prime}\right) \wedge \neg N\left(c \overline{p^{\prime}}\right)\right) \vee$ $\left(\neg N\left(c p^{\prime}\right) \wedge N\left(c \overline{p^{\prime}}\right)\right)$ and replacing each $\neg N c$ in $\delta_{\mathrm{zg}}$ by $\neg N\left(c p^{\prime}\right) \wedge \neg N\left(c \overline{p^{\prime}}\right)$. Then take its disjunctive normal form with $N c$ and $\neg N c$ as atomic formulas.

Now it should not be too hard to see that $\psi$ is provably equivalent to $\varphi$ as each of $\psi_{i}$ is provably equivalent to the respective part of $\varphi$ if it exists, or to $\top$ otherwise. In particular, to see that $\psi_{4} \dashv \vdash \delta_{\mathrm{zg}}$ and $\psi_{5} \dashv \vdash \delta_{\mathrm{z} \overline{\mathrm{g}}}$, use Lemma 4.10. Then, let $\varphi^{\prime}$ be the result of distributing the outermost conjunction over $\psi_{1}$ through $\psi_{5}$ over the disjunctions in them. Clearly $\varphi^{\prime}$ is now a disjunction of state descriptions over $\mathbf{p}^{\prime}$ with degree $l^{\prime}$, and $\varphi^{\prime}$ is provably equivalent to $\varphi$.

Lemma 4.14 Any formula $\varphi \in \mathscr{L}$ Пzg with $\mathbf{p}$ being its set of free variables is provably equivalent in $\mathrm{KD} 4{ }^{\forall} 5$ Пzg to a disjunction of state descriptions over $\mathbf{p}$. 
Proof By induction. Since we are only after provable equivalence, we can pretend that our language has $\vee, \widehat{B}$, and $\exists$ as primitives. For the base cases, note that:

- every propositional variable $p$ in Prop is a partial state description in $\{p\}$ with degree 0;

- $\quad T$ is a partial state description in \{\} with degree 0 ;

- since $z \dashv$ z $(z \mathrm{~g} \vee \mathrm{z} \overline{\mathrm{g}}), \mathrm{z}$ is equivalent to a disjunction of two partial state descriptions over \{\} with degree 0 ;

- since $\vdash \mathrm{g} \rightarrow \mathrm{z}$ and $\mathrm{g} \dashv \vdash \mathrm{zg}, \mathrm{g}$ is also provably equivalent to a disjunction of partial state descriptions over \{\} with degree 0 .

Hence we can apply the previous lemma to clear the base cases.

Now suppose $\varphi=\varphi_{1} \vee \varphi_{2}$, and let $\mathbf{p}_{1}$ and $\mathbf{p}_{2}$ be the set of free variables in $\varphi_{1}$ and $\varphi_{2}$, respectively. Then $\mathbf{p}=\mathbf{p}_{1} \cup \mathbf{p}_{2}$ is the set of free variables of $\varphi$. By the induction hypothesis, there is a disjunction $\psi_{1}$ of state descriptions over $\mathbf{p}_{1}$ with some degree $l_{1}$ provably equivalent to $\varphi_{1}$ and a disjunction $\psi_{2}$ of state descriptions over $\mathbf{p}_{2}$ with some degree $l_{2}$ provably equivalent to $\varphi_{2}$. Without loss of generality, we can assume that $l_{1} \geq l_{2}$. Now we first use the previous Lemma 4.13 to turn $\psi_{1}$ and $\psi_{2}$ into disjunctions of state descriptions over $\vec{p}$ with degree $l_{1}$ and obtain $\alpha$ and $\beta$. Then $\alpha \vee \beta$ is the formula we need in this case.

For the negation case, suppose $\varphi=\neg \psi$ with $\mathbf{p}$ being the set of free variables in $\psi$. Then $\mathbf{p}$ is also the set of free variables in $\varphi$. Using the induction hypothesis, let $\alpha$ be a disjunction of state descriptions over $\mathbf{p}$ with some degree $l$ that is provably equivalent to $\psi$. Then using Lemma 4.13 , let $\beta$ be a disjunction of state descriptions over $\mathbf{p}$ with degree $l$ that is provably equivalent to $T$. Then let $\gamma$ be the disjunction of the state descriptions over $\mathbf{p}$ with degree $l$ that are in $\beta$ but not in $\alpha$. Then $\gamma$ is a disjunction of state descriptions over $\mathbf{p}$ with degree $l$ that is provably equivalent to $\varphi$.

Now suppose $\varphi=\widehat{B} \psi$ with $\mathbf{p}$ being the set of free variables in $\psi$ and hence also $\varphi$. By the induction hypothesis, $\psi$ is provably equivalent to a disjunction of state descriptions $\bigvee_{i \in I} \psi_{i}$. Then $\widehat{\mathrm{B}} \psi-1 \widehat{\mathrm{B}} \bigvee_{i \in I} \psi_{i} \dashv \vdash \bigvee_{i \in I} \widehat{\mathrm{B}} \psi_{i}$. Hence, we only need to show that for each state description $\psi_{i}, \widehat{\mathrm{B}} \psi_{i}$ is equivalent to a partial state description, which can then be turned to a disjunction of state descriptions. Let $\psi_{i}=$ $b \wedge c \wedge d$ so that $b \in\{\bar{z}, \mathrm{zg}, \mathrm{z} \overline{\mathrm{g}}\}, c \in 2^{\mathbf{p}}$, and $d$ is the modal part of $\psi_{i}$. By Lemma 4.2, $\psi_{i} \dashv \vdash(b \wedge c \wedge \mathrm{B} d)$ since $d$ is fully modalized. Then it is a standard exercise of modal logic to show that $\widehat{\mathrm{B}} \psi_{i} \dashv \vdash \widehat{\mathrm{B}}(b \wedge c) \wedge \mathrm{B} d$, which is then provably equivalent to $\widehat{\mathrm{B}}(b \wedge c) \wedge d$. Now there are three cases:

- if $b=\overline{\mathrm{z}}$, then $\widehat{\mathrm{B}}(b \wedge c)$ is just $\langle\overline{\mathrm{z}}\rangle c$. But then $\langle\overline{\mathrm{z}}\rangle c \wedge d$ is a partial state description, as $\langle\bar{z}\rangle c$ is a $\mathscr{L}[\bar{z}] \Pi$ formula and can be included in the $\bar{z}$ part.

- if $b=\mathrm{zg}$, then $\widehat{\mathrm{B}}(b \wedge c) \dashv \vdash\langle\mathrm{z}\rangle(\mathrm{g} \wedge c)$. But $\langle\mathrm{z}\rangle(g \wedge c) \dashv \vdash M_{1} c$, and $M_{1} c \wedge d$ can be turned into a partial state description, as there is a $Q_{i} c / M_{l} c$ formula in $d$, and $M_{1} c$ can be merged in to that formula, resulting in $\perp$ or the original $d$.

- if $b=\mathrm{z} \overline{\mathrm{g}}$, then $\widehat{\mathrm{B}}(b \wedge c) \dashv \vdash\langle\mathrm{z}\rangle(\overline{\mathrm{g}} \wedge c)$. But $\langle\mathrm{z}\rangle(\overline{\mathrm{g}} \wedge c) \dashv \vdash N$ and hence can be merged into $d$.

In sum, $\widehat{\mathbf{B}} \psi_{i}$ is provably equivalent to a partial state description missing a choice of a zg part and a propositional truth part. 
For $\exists$, like $\widehat{\mathrm{B}}$, we only need to show that $\exists q \psi_{i}$ is provably equivalent to a partial state description over $\mathbf{p} \backslash\{q\}$ where $\psi_{i}$ is a state description over $\mathbf{p}$. The case where $q \notin \vec{p}$ is trivial so we assume here that $q \in \mathbf{p}$. Let $\psi_{i}$ be $b \wedge c \wedge \delta_{\overline{\mathrm{z}}} \wedge \delta_{\mathrm{zg}} \wedge \delta_{\mathrm{z} \overline{\mathrm{g}}}$, where $b \in\{\overline{\mathrm{z}}, \mathrm{zg}, \mathrm{z} \overline{\mathrm{g}}\}, c \in 2^{\mathbf{p}}, \delta_{\overline{\mathrm{z}}}$ is the $\overline{\mathrm{z}}$ part of $\psi_{i}$ (a $\mathscr{L}[\overline{\mathrm{z}}] \Pi$ formula), $\delta_{\mathrm{zg}}$ is the zg part, and $\delta_{\mathrm{z} \overline{\mathrm{g}}}$ is the $\mathrm{z} \overline{\mathrm{g}}$ part. As noted in Lemma 4.12, $\delta_{\overline{\mathrm{z}}}, \delta_{\mathrm{zg}}$, and $\delta_{\mathrm{z} \overline{\mathrm{g}}}$ are restricted by $\overline{\mathrm{z}}$, $z g$, and $z \bar{g}$ respectively. Then by repeated use of Lemma 4.11, we have the following cases:

- If $b=\overline{\mathrm{z}}$, then $\exists q \psi_{i} \dashv \vdash \exists q\left(\overline{\mathrm{z}} \wedge c \wedge \delta_{\overline{\mathrm{z}}}\right) \wedge \exists q \delta_{\mathrm{zg}} \wedge \exists q \delta_{\mathrm{z} \overline{\mathrm{g}}}$.

- If $b=\mathrm{zg}$, then $\exists q \psi_{i} \dashv \vdash \exists q \delta_{\overline{\mathrm{z}}} \wedge \exists q\left(\mathrm{zg} \wedge c \wedge \delta_{\mathrm{zg}}\right) \wedge \exists q \delta_{\mathrm{zg}}$.

- If $b=\mathrm{z} \overline{\mathrm{g}}$, then $\exists q \psi_{i} \dashv \vdash \exists q \delta_{\overline{\mathrm{z}}} \wedge \exists q \delta_{\mathrm{zg}} \wedge \exists q\left(\mathrm{z} \overline{\mathrm{g}} \wedge c \wedge \delta_{\mathrm{zg}}\right)$.

Hence what remains to be shown is that in each of these three cases, the three conjuncts on the right-hand side of the $-\vdash$ claim are provably equivalent to a $\bar{z}$ part, a zg part, and a $z \bar{g}$ part, possibly with a corresponding choice of $z g$ and a propositional truth $c$, respectively.

First consider the two possibilities $\exists q \delta_{\bar{z}}$ and $\exists q\left(\overline{\mathbf{z}} \wedge c \wedge \delta_{\overline{\mathrm{z}}}\right)$. We need to show that they are provably equivalent to some $\bar{z}$ part. Now $\exists q \delta_{\bar{z}}$ is already a $\mathscr{L}[\bar{z}] \Pi$ formula and thus a $\bar{z}$ part, so there is nothing further to show. For $\exists q\left(\bar{z} \wedge c \wedge \delta_{\bar{z}}\right)$, depending on whether $q$ appears in $c$ positively or negatively, we have two cases (the $f$ below is the result of excluding the literal of $q$ in $c$ ).

- If $q$ appears positively, we have $\exists q\left(\overline{\mathbf{z}} \wedge f \wedge q \wedge \delta_{\bar{z}}\right)$. This is provably equivalent to $\bar{z} \wedge f \wedge \exists q \delta_{\bar{z}}$. The direction from left to right is trivial. For the other direction, if $q$ is not free in $\delta_{\overline{\mathbf{z}}}$, it is also trivial. So assume now that $q$ is free in $\delta_{\overline{\mathbf{z}}}(q)$. First instantiate $\exists q \delta_{\overline{\mathbf{z}}}(q)$ with a fresh $a$ and obtain $\delta_{\overline{\mathbf{z}}}(a)$. Now recall that $\overline{\mathrm{z}} \dashv \vdash \exists r(r \wedge$ $\mathrm{B} \neg r)$. Since we already have $\bar{z}$, we can now instantiate with a fresh propositional variable $b$ and get $b \wedge \mathrm{B} \neg b$. By Lemma 4.9, we derive $\delta_{\overline{\mathbf{z}}}(a) \leftrightarrow \delta_{\overline{\mathbf{z}}}(a \vee b)$ (recall that $\delta_{\overline{\mathbf{z}}}(a)$ is in $\left.\mathscr{L} \mathrm{B} \Pi z \mathrm{zg}\right)$, and hence also $\delta_{\overline{\mathbf{z}}}(a \vee b)$. But $\vdash b \rightarrow(a \vee b)$, so we also obtain $a \vee b$. Then $a \vee b$ witnesses $\exists q\left(q \wedge \delta_{\bar{z}}(q)\right)$. Summing up the process, we have shown that $\vdash\left(\overline{\mathrm{z}} \wedge \exists q \delta_{\overline{\mathrm{z}}}\right) \rightarrow \exists q\left(q \wedge \delta_{\overline{\mathrm{z}}}\right)$. Then it is easy to see that $\vdash\left(\overline{\mathrm{z}} \wedge f \wedge \exists q \delta_{\overline{\mathrm{z}}}\right) \rightarrow \exists q\left(\overline{\mathrm{z}} \wedge f \wedge q \wedge \delta_{\overline{\mathrm{z}}}\right)$ since $q$ does not appear in $\overline{\mathrm{z}}$ and $f$.

- $\exists q\left(\overline{\mathrm{z}} \wedge f \wedge \neg q \wedge \delta_{\overline{\mathrm{z}}}\right)$. This is very similar to the previous case. We only need to prove the direction from $\overline{\mathrm{z}} \wedge f \wedge \exists \delta_{\overline{\mathbf{z}}}$ to $\exists q\left(\overline{\mathrm{z}} \wedge f \wedge \neg q \wedge \delta_{\overline{\mathbf{z}}}\right)$ and in fact only that $\vdash\left(\overline{\mathbf{z}} \wedge \exists q \delta_{\overline{\mathbf{z}}}\right) \rightarrow \exists q\left(\neg q \wedge \delta_{\overline{\mathbf{z}}}\right)$. We can also assume that $q$ is free in $\delta_{\overline{\mathbf{z}}}(q)$. Instantiating $\exists q \delta_{\bar{z}}$ and $\exists r(r \wedge B \neg r)$ (equivalent to $\bar{z}$ ) with fresh $a$ and $b$, we get $\delta_{\overline{\mathbf{z}}}(a)$ and $b \wedge \mathrm{B} \neg b$. By Lemma 4.9, we get $\delta_{\overline{\mathbf{z}}}(a \wedge \neg b)$. Also, $\vdash b \rightarrow \neg(a \wedge \neg b)$. Hence $a \wedge \neg b$ witnesses $\exists q\left(\neg q \wedge \delta_{\bar{z}}(q)\right)$, and we are done in this case.

So, the two formulas involving $\delta_{\bar{z}}$ are indeed provably equivalent to formulas that can serve as the $\bar{z}$ part of some state description over $\mathbf{p} \backslash\{q\}$.

For the cases involving $\delta_{\mathrm{zg}}$ and $\delta_{\mathrm{z} \overline{\mathrm{g}}}$, note that both of them are conjunctions of formulas that are restricted to one of $c \in 2^{\mathbf{p}}$. Considering this, we can push $\exists q$ further down, with results in the following cases where again $f$ is the result of restricting $c$ to literals using only things in $\mathbf{p} \backslash\{q\}$ :

- $\exists q\left(Q_{i} / M_{l}(f q) \wedge Q_{j} / M_{l}(f \bar{q})\right)$,

- $\exists q\left(\mathrm{zg} \wedge f \wedge \pm q \wedge Q_{i} / M_{l}(f q) \wedge Q_{j} / M_{l}(f \bar{q})\right)$, 
- $\exists q( \pm N(f q) \wedge \pm N(f \bar{q}))$,

- $\exists q(\mathrm{z} \overline{\mathrm{g}} \wedge f \wedge \pm q \wedge \pm N(f q) \wedge \pm N(f \bar{q}))$.

They are all addressed in Lemma 4.10.

Now Lemma 4.7 follows from the previous lemma. Too see this, observe first that the modal parts of any state description are $\mathscr{L}$ B $\Pi z g$ formulas. Further, when there are no free variables, the propositional truth part will be $T$ in any state description. So a state description over \{\} can be seen as simply a conjunction of one of $\bar{z}, z \mathrm{~g}, \mathrm{z} \overline{\mathrm{g}}$, and a $\mathscr{L}$ B zg formula. Then for any sentence $\varphi$ in $\mathscr{L} \Pi$, since it has no free variables, it is provably equivalent in $\mathrm{KD} 4^{\forall} 5 \Pi \mathrm{zg}$ to a disjunction $\bigvee_{i \in I}\left(a_{i} \wedge b_{i}\right)$ such that for all $i \in I, a_{i} \in\{\bar{z}, \mathrm{zg}, \mathrm{z} \overline{\mathrm{g}}\}$ and $b_{i} \in \mathscr{L} \mathrm{B}$ Izg. But then we only need to extract the $a_{i}$ 's according to what they are. Formally, letting $I_{\bar{z}}=\left\{i \in I \mid a_{i}=\bar{z}\right\}, I_{\mathrm{zg}}=\{i \in I \mid$ $\left.a_{i}=\mathrm{zg}\right\}$, and $I_{\mathrm{z} \overline{\mathrm{g}}}=\left\{i \in I \mid a_{i}=\mathrm{z} \overline{\mathrm{g}}\right\}, \varphi \dashv \vdash\left(\overline{\mathrm{z}} \wedge \bigvee_{i \in I_{\overline{\mathrm{z}}}} b_{i}\right) \vee\left(\mathrm{zg} \wedge \bigvee_{i \in I_{\mathrm{zg}}} b_{i}\right) \vee$ $\left(\mathrm{z} \overline{\mathrm{g}} \wedge \bigvee_{i \in I_{\mathrm{z} \overline{\mathrm{g}}}} b_{i}\right)$. This formula is in the required form in Lemma 4.7.

\subsection{Quotients of Complete Boolean Algebras}

In this subsection we prove Lemma 4.8. The main idea is to show that every Boolean algebra is elementarily equivalent to a $\kappa$-field-of-sets for a large enough cardinal $\kappa$ to be speficied later and then invoke a theorem saying that every $\kappa$-field-of-sets can be realized as a quotient of a complete Boolean algebra for large enough $\kappa$. To show that every Boolean algebra is elementarily equivalent to a $\kappa$-field-of-sets, we show that every Tarski invariant, which fully describes the first-order properties of Boolean algebras, is realized by a $\kappa$-field-of-sets.

First we define $\kappa$-regular subalgebras and $\kappa$-field-of-sets.

Definition 4.14 A Boolean algebra $B$ is a $\kappa$-regular subalgebra of $C$ if $B$ is a subalgebra of $C$ and for any $X \subseteq B$ with $|X|<\kappa$, whenever $\bigwedge_{C} X$ exists, it is also in $B$. We write $B \preccurlyeq{ }_{\kappa} C$ for $B$ being a $\kappa$-regular subalgebra of $C$.

We say an embedding $f: B \hookrightarrow C$ is $\kappa$-regular if for every $X \subseteq B$ such that $|X|<\kappa$, whenever $\bigwedge_{C} f[X]$ exists, $\bigwedge_{B} X$ also exists and $f\left(\bigwedge_{B} X\right)=\bigwedge_{C} f[X]$. Or equivalently, $f$ is $\kappa$-regular if the image of $f$ is a $\kappa$-regular subalgebra of $C$. We write $f: B \hookrightarrow_{\kappa} C$ when $f$ is a $\kappa$-regular embedding from $B$ to $C$ and write $B \hookrightarrow{ }_{\kappa} C$ when there is such a $\kappa$-regular embedding.

Definition 4.15 A Boolean algebra $B$ is a $\kappa$-field-of-sets if there is a set $D$ such that $B \hookrightarrow_{\kappa} \wp(D)$. Here $\wp(D)$ is the powerset algebra of $D$.

Proposition 4.4 For any cardinal $\kappa$, the property of being a $\kappa$-field-of-sets is closed taking $\kappa$-regular subalgebras and is closed under taking arbitrary direct product.

Proof First, clearly, if $A \hookrightarrow_{\kappa} B$ and $B \hookrightarrow_{\kappa} C$, then $A \hookrightarrow_{\kappa} C$. Hence if $A \preccurlyeq{ }_{\kappa} B$ and $B \hookrightarrow_{\kappa} \wp(D)$, then $A \hookrightarrow_{\kappa} \wp(D)$. Thus $\kappa$-field-of-sets is closed under taking $\kappa$-regular subalgebras. 
Now consider an indexed set $\left\{B_{i}\right\}_{i \in I}$ of $\kappa$-field-of-sets with $f_{i}: B_{i} \hookrightarrow_{\kappa} \wp\left(D_{i}\right)$ for each $i \in I$. Then it is not hard to see that $\Pi_{i \in I} B_{i} \hookrightarrow_{\kappa} \Pi_{i \in I} \wp\left(D_{i}\right)$. This is because, letting $\pi_{i}$ be the natural projection map from $\Pi_{i} B_{i}$ to $B_{i}$, for every $X \subseteq \Pi_{i} B_{i}$, $\bigwedge X=\left\langle\bigwedge \pi_{i}[X]\right\rangle_{i \in I}$, if any side of this equation exists. In other words, meets can be computed componentwisely. But $\Pi_{i \in I} \wp\left(D_{i}\right)$ is isomorphic to $\wp\left(\bigcup_{i \in I}\left(\{i\} \times D_{i}\right)\right)$. Hence $\Pi_{i \in I} B_{i}$ is also a $\kappa$-field-of-sets.

Due to the fact that we need to deal with zg-algebras instead of just Boolean algebras, sometimes we need to make sure that the cokernels of the quotient maps we use have a trivial meet. We now introduce notations for this and prove two lemmas about it.

Definition 4.16 We say a surjective homomorphism $f: A \rightarrow B$ is meet-trivial if its cokernel $f^{-1}\left(\top_{B}\right)$ has a trivial meet: $\bigwedge f^{-1}\left(\top_{B}\right)$ is $\perp_{A}$. We write $f: A \leftrightarrow B$ when $f: A \rightarrow B$ and $f$ is meet-trivial, and we write $A \nrightarrow B$ when there is a meettrivial surjective homomorphism from $A$ to $B$. In the later case, we also say that $B$ is a meet-trivial homomorphic image of $A$.

Proposition 4.5 For any Boolean algebras $A, B$, and $C$, if $f: A \rightarrow B$ and $g: B \odot »$ $C$, then $(g \circ f): A \odot \nrightarrow C$.

Proof Let $f: A \rightarrow B$ and $g: B \leftrightarrow C$ be given. To show that $g \circ f: A \leftrightarrow C$, by definition, we only need to show that $\bigwedge F=\perp_{A}$ where $F=(g \circ f)^{-1}\left(\top_{C}\right)$. Suppose not and let $a$ be a non-trivial lower bound of $F$ in $A$. Then first we can show that $f(a) \neq \perp_{B}$ since if otherwise $f(a)=\perp_{B}$, then $f(\neg a)=\top_{B}$, meaning that $g(f(\neg a))=\top_{C}$ and that $\neg a \in F$, which obviously contradicts the assumption that $a$ is below everything, in particular $\neg a$, in $F$ and that $a \neq \perp_{A}$. Since $f$ is a homomorphism, $f(a)$ is a lower bound of $f[F]$. Then we only need to note that $g^{-1}\left(\top_{C}\right)=f[F]$, and hence $f(a)$ is a non-trivial lower bound of $g^{-1}\left(\top_{C}\right)$, contradicting $g: B \leftrightarrow \nrightarrow C$.

Proposition 4.6 For any $\kappa$-field-of-sets $B$ where $\kappa$ is a regular cardinal, meaning that the cofinality $\operatorname{cf}(\kappa)=\kappa$, there is a $\kappa$-field-of-sets $C$ such that $C \leftrightarrow B B$.

Proof Let $\kappa$ and $B$ be given as above. Then consider the following subset of $B^{\kappa}=$ $\Pi_{i<\kappa} B$ :

$$
C=\left\{f \in B^{\kappa} \mid \exists \alpha \in \kappa, \forall \beta \in \kappa, \text { if } \beta \geq \alpha \text { then } f(\beta)=f(\alpha)\right\} .
$$

The set $C$ colletcs what we may call the eventually constant elements in $B^{\kappa}$. For every $f \in C$, let $\lim f$ be the limit of $f$ defined in the obvious way. Now we show that $C$ with operations inherited from $B^{\kappa}$ is a $\kappa$-regular subalgebra of $B^{\kappa}$.

1. $C$ is closed under negation. This is trivial.

2. $C$ is closed under taking meets of sets of cardinality smaller than $\kappa$. Take any $\left\{f_{i}\right\}_{i \in I} \subseteq C$ with $|I|<\kappa$. Let $\alpha_{i}$ for each $i \in I$ be the smallest ordinal in $\kappa$ such that for any $\beta$ such that $\kappa>\beta \geq \alpha_{i}, f_{i}(\beta)=f_{i}\left(\alpha_{i}\right)$. Then let $f=\bigwedge f_{i}$ 
in $B^{\kappa}$. Now because $\operatorname{cf}(\kappa)=\kappa, \alpha=\sup _{i \in I} \alpha_{i}<\kappa$. Thus for any $\beta$ such that $\kappa>\beta \geq \alpha$ and any $i \in I, f_{i}(\beta)=f_{i}\left(\alpha_{i}\right)=f_{i}(\alpha)$. Hence for any $\kappa>\beta \geq \alpha$,

$$
f(\beta)=\bigwedge_{i \in I} f_{i}(\beta)=\bigwedge_{i \in I} f_{i}(\alpha)=f(\alpha) .
$$

Then we know that $f \in C$.

This also shows that for any set $\left\{f_{i}\right\}_{i \in I} \subseteq C$ with $|I|<\kappa, \lim \bigwedge_{i \in I} f_{i}=$ $\bigwedge_{i \in I} \lim f_{i}$.

Using Proposition 4.4, $C$ is now also a $\kappa$-field-of-sets since $C \preccurlyeq{ }_{\kappa} B^{\kappa}$ and $B$ is a $\kappa$-field-of-sets. Now consider the set $F=\left\{f \in C \mid \lim f=\top_{B}\right\}$. Observe that $F$ is a filter in $C$. Also, $\bigwedge F=\perp_{C}$. To see this, consider the sequence $\left\langle f_{i}\right\rangle_{i \in \kappa}$ defined by

$$
f_{i}(\alpha)= \begin{cases}\perp_{B} & \alpha<i \\ \top_{B} & \alpha \geq i .\end{cases}
$$

Each $f_{i}$ is in $F$, yet the only $f \in C$ that is below all the $f_{i}$ 's is the constantly $0_{B}$ function, which is $\perp_{C}$.

Now note that lim as a function from $C$ to $B$ is a surjective homomorphism and $\lim ^{-1}\left(\top_{B}\right)=F$. Hence lim : $C \odot \mapsto B$.

Now we start to show that for every non-trivial Boolean algebra $A$, there is a $\left(2^{\omega_{0}}\right)^{+}$-field-of-sets $B$ which is elementarily equivalent to $A$. To this end, we first recall the Tarski invariants.

Definition 4.17 For any Boolean algebra $B$, call an element $b \in B$ atomic if $b$ is the join of the atoms below it, and atomless if there are no atoms below it. If an element is the join of an atomic element and an atomless element, we call it separable. Denote the set of separable element in $B$ by $S(B)$. It is easy to see that $S(B)$ is an ideal, which is generated by the atomic and atomless elements.

Then for any non-trivial Boolean algebra $B$, we can define a sequence of Boolean algebras:

$$
B^{(0)}=B, B^{(i+1)}=B^{(i)} / S\left(B^{(i)}\right) .
$$

With the above sequence, define $\operatorname{Inv}(B)$ for every non-trivial Boolean algebra $B$ as follows:

$$
\begin{aligned}
& m(B)= \begin{cases}k & \text { if } B^{(k)} \text { is non-trivial and } B^{(k+1)} \text { is trivial } \\
\infty & \text { if for all } k \in \omega, B^{(k)} \text { is non-trivial. }\end{cases} \\
& n_{0}(B)= \begin{cases}\infty & \text { if } m(B) \in \mathbb{N} \text { and } B^{(m(B))} \text { has infinitely many atoms } \\
l & \text { if } m(B) \in \mathbb{N} \text { and } B^{(m(B))} \text { has } l \in \mathbb{N} \text { many atoms. }\end{cases} \\
& n(B)= \begin{cases}0 & \text { if } m(B) \notin \mathbb{N} \\
n_{0}(B) & \text { if } m(B) \in \mathbb{N} \text { and } B^{(m(B))} \text { is atomic } \\
-n_{0}(B) & \text { if } m(B) \in \mathbb{N} \text { and } B^{(m(B))} \text { is not atomic. }\end{cases} \\
& \operatorname{Inv}(B)=\langle m(B), n(B)\rangle .
\end{aligned}
$$


We also define $\operatorname{Inv}(B)=\langle-1,0\rangle$ when $B$ is trivial. Finally let Inv be the set of all possible invariant, i.e., $\operatorname{Inv}=\{\operatorname{Inv}(B) \mid B$ a Boolean algebra $\}$.

Proposition 4.7 For any two Boolean algebras $A$ and $B$, they are elementarily equivalent if and only if $\operatorname{Inv}(A)=\operatorname{Inv}(B)$. In fact, for any two Boolean algebras with extra distinguished elements, $\left\langle A, a_{1}, a_{2}, \cdots a_{n}\right\rangle,\left\langle B, b_{1}, b_{2}, \cdots b_{n}\right\rangle$, they are elementarily equivalent in the first-order language of Boolean algebras extended with $n$ constants to be interpreted by the corresponding distinguished elements if and only if for each $f \in 2^{n}, \operatorname{Inv}\left(\left.A\right|_{f[\mathbf{a}]}\right)=\operatorname{Inv}\left(\left.B\right|_{f[\mathbf{b}]}\right)$. Here for any $f \in 2^{n}, f[\mathbf{a}]$ is defined as the element $\bigwedge_{i \in f^{-1}(1)} a_{i} \wedge \bigwedge_{i \in f^{-1}(0)} \neg a_{i}$ and $f[\mathbf{b}]$ is defined similarly.

\section{Proof See Chap.5.5 of [52].}

Hence our goal now is to construct a $\left(2^{\omega_{0}}\right)^{+}$-field-of-sets $B$ for each invariant $c \in \operatorname{Inv}$ such that $\operatorname{Inv}(B)=c$. To start, we need at least an atomic and an atomless $\kappa$-field-of-sets. An atomic $\kappa$-field of sets can be easily found, such as the Boolean algebra of two elements. Now we construct an atomless $\kappa$-field-of-sets.

Proposition 4.8 For each regular infinite cardinal $\kappa$, there exists an atomless $\kappa$ field-of-sets $L$.

Proof We construct a $\kappa$-field-of-sets in the powerset algebra of $\{0,1\}^{\kappa}$. For any $f, g \in\{0,1\}^{\kappa}$ and $i \in \kappa$, write $f={ }_{i} g$ when $f(j)=g(j)$ for all $j<i$. Also let $[f]_{i}$ be the equivalence class that $f$ is in under $=_{i}$, i.e. $\left\{g \in\{0,1\}^{\kappa} \mid g=_{i} f\right\}$.

Then it is not hard to see that the set of all subsets of $\{0,1\}^{\kappa}$ that are closed under $={ }_{i}$ for some $i \in \kappa$ forms an atomless $\kappa$-field-of-sets under the inherited complementation and intersection.

- Both empty set and $\{0,1\}^{\kappa}$ are closed under $=_{1}$.

- Clearly if $X$ is closed under $={ }_{i}$, then $\{0,1\}^{\kappa} \backslash X$ is also closed under $={ }_{i}$.

- For any family of $\kappa_{0}<\kappa$ many sets $\left\{X_{i}\right\}_{i \in \kappa_{0}}$ such that each $X_{i}$ is closed under $=\lambda_{i}$ where $\lambda_{i} \in \kappa$, consider their intersection. Let $\mu=\sup _{i \in \kappa_{0}} \lambda_{i}$. By the regularity of $\kappa, \mu \in \kappa$, and obviously each $X_{i}$ is also closed under $={ }_{\mu}$ since $={ }_{\alpha}$ refines $=\beta$ if $\alpha \geq \beta$. Then the intersection $\bigcap\left\{X_{i} \mid i \in \kappa_{0}\right\}$ is also closed under $=\mu$.

- For any non empty $X \subseteq\{0,1\}^{\kappa}$ that is closed under $={ }_{\lambda}$, pick $f \in X$ and then we can easily split $[f]_{\lambda} \subseteq X$ into two non-empty parts: $\left\{g \in[f]_{\lambda} \mid g(\lambda+1)=0\right\}$ and $\left\{g \in[f]_{\lambda} \mid g(\lambda+1)=1\right\}$. Both parts are non-empty subsets of $X$ and are closed under $=\lambda+1$. So $X$ is not an atom in the Boolean algebra we construct.

Now fix $\kappa$ as an infinite regular cardinal, 2 a two-element Boolean algebra, and $L$ the atomless $\kappa$-field-of-sets constructed above. The next step is to show that for every $\kappa$-field-of-sets $B$, there is a $U(B)$ which is also a $\kappa$-field-of-sets, and moreover $U(B) / S(U(B)) \cong B$. Since we are constructing a $\kappa$-field-of-sets that has $B$ as a homomorphic image with some requirement on the kernel of the homomorphism, the construction here is very similar to the one we did in Proposition 4.6. 
For a $\kappa$-field-of-sets $B$, we construct $U(B)$ as follows. First, since $B$ is a $\kappa$-fieldof-sets, without loss of generality, we can assume that $B \preccurlyeq_{\kappa} \wp(\rho)$ with $\rho$ a large enough cardinal. Then we have $e: B \hookrightarrow_{\kappa}(2 \times L)^{\rho}$ where $e$ is such that for all $b \in B$ and $\lambda<\rho, e(b)(\lambda)=\top_{2 \times L}$ if and only if $\lambda \in b$, and otherwise $e(b)(\lambda)=\perp_{\mathbf{2} \times L}$. In other words, $e(b)$ is the characteristic function of $b$ using $\left\{\perp_{\mathbf{2} \times L}, \top_{\mathbf{2} \times L}\right\}$ instead of $\{0,1\}$ as the codomain. By Proposition $4.4,(2 \times L)^{\rho}$ is a $\kappa$-field-of-sets. Now, as in the proof of Proposition 4.6, we can now define the set of eventually constant functions $C=\left\{f \in\left((2 \times L)^{\rho}\right)^{\kappa} \mid \exists \alpha<\kappa \forall \beta<\kappa, \beta>\alpha \Rightarrow f(\beta)=f(\alpha)\right\}$. Then we know that $C \preccurlyeq_{\kappa}\left((2 \times L)^{\rho}\right)^{\kappa}$ and hence is a $\kappa$-field-of-sets, and in addition $\lim : C \Theta \nrightarrow(2 \times L)^{\rho}$. However, since we need a $\kappa$-field-of-sets with $B$, not $(2 \times L)^{\rho}$, as its homomorphic image, we need to take a $\kappa$-regular subalgebra of $C$. Indeed, we only need to take $U(B)=\lim ^{-1}(e[B])$. Essentially, $U(B)$ is the pullback of $e$ and lim. This is illustrated by the following commutative diagram:

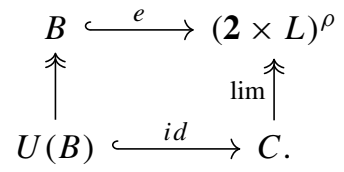

Lemma 4.15 For any $\kappa$-field-of-sets $B, U(B)$ as defined above is also a $\kappa$-field-ofsets, and $U(B) / S(U(B)) \cong B$.

Proof To show that $U(B)$ is a $\kappa$-field-of-sets, it is enough to show that $U(B) \preccurlyeq_{\kappa}$ $\left((2 \times L)^{\rho}\right)^{\kappa}$. That is, we only need to show that for all $X \subseteq U(B)$ such that $|X|<\kappa$, $\bigwedge_{\left((2 \times L)^{\rho}\right)^{\kappa}} X$ is also in $U(B)$ (it always exists as $\left((2 \times L)^{\rho}\right)^{\kappa}$ is a $\kappa$-field-of-sets). To show that $\bigwedge_{\left((2 \times L)^{\rho}\right)^{\kappa}} X \in U(B)$, we only need to show that $\lim \bigwedge_{\left((2 \times L)^{\rho}\right)^{\kappa}} X \in$ $e[B]$. Since $\kappa$ is regular, $\lim \bigwedge_{\left((2 \times L)^{\rho}\right)^{\kappa}} X=\bigwedge_{(2 \times L)^{\rho}} \lim [X]$. Since $X \subseteq U(B)$, $\lim [X] \subseteq e[B]$. Since $|X|<\kappa,|\lim [X]|<\kappa$. Then indeed $\bigwedge_{(2 \times L)^{\rho}} \lim [X] \in e[B]$ since $e$ is a $\kappa$-regular embedding, and hence $e[B] \preccurlyeq \preccurlyeq_{\kappa}(2 \times L)^{\rho}$.

To show that $U(B) / S(U(B)) \cong B$, it is enough to show that the kernel of lim, $\lim ^{-1}\left(\perp_{\left.(2 \times L)^{\rho}\right)}\right.$, is precisely $S(U(B))$, the set of separable elements in $U(B)$. To this end, note first that in both $(2 \times L)^{\rho}$ and $\left((2 \times L)^{\rho}\right)^{\kappa}$, the join of atoms exists and can be easily described. Let $\mathbf{a}$ be the constantly $\left\langle\top_{\mathbf{2}}, \perp_{L}\right\rangle$ function in $(\mathbf{2} \times L)^{\rho}$ and $f_{\mathbf{a}}$ be the constantly a function in $\left((2 \times L)^{\rho}\right)^{\kappa}$. Then clearly $\mathbf{a}$ is the join of atoms in $(2 \times L)^{\rho}$, and $f_{\mathbf{a}}$ is the join of atoms in $\left((2 \times L)^{\rho}\right)^{\kappa}$. Similarly, let $\mathbf{l}$ be the constantly $\left\langle\perp_{\mathbf{2}}, \top_{L}\right\rangle$ function in $(2 \times L)^{\rho}$ and $f_{\mathbf{I}}$ be the constantly $\mathbf{l}$ function in $\left((\mathbf{2} \times L)^{\rho}\right)^{\kappa}$. Then $\mathbf{l}$ is the join of atomless elements in $(2 \times L)^{\rho}$, and $f_{\mathbf{l}}$ is the join of atomless elements in $\left((2 \times L)^{\rho}\right)^{\kappa}$.

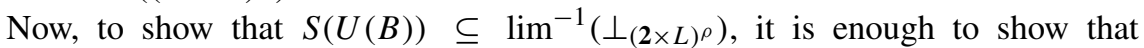
every atomic and every atomless elements in $U(B)$ are in $\lim ^{-1}\left(\perp_{(2 \times L)^{\rho}}\right)$ since the $S(U(B))$ is the ideal generated by those elements and a kernel is always an ideal. Let $f$ be an atomic element in $U(B)$. First, we claim that $f \leq f_{\mathbf{a}}$. Suppose not. Then there would be $i<\kappa, j<\rho$ such that $f(i)(j) \wedge \top_{L}$ is non-trivial. Let $g$ be the function in $\left((2 \times L)^{\rho}\right)^{\kappa}$ such that $g\left(i^{\prime}\right)\left(j^{\prime}\right)=\perp_{\mathbf{2} \times L}$ unless $i^{\prime}=i$ and $j^{\prime}=j$, in which case $g\left(i^{\prime}\right)\left(j^{\prime}\right)=f(i)(j) \wedge \top_{L}$. Then $g \leq f, \lim g=\perp_{(\mathbf{2} \times L)^{\rho}}$, and hence $g \in U(B)$. But obviously $g$ is atomless in $U(B)$ since we can simply keep decreasing $g(i)(j)$ using the fact that $L$ is atomless, and the resulting function's limit is still 


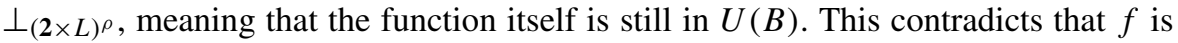
atomic. So $f \leq f_{\mathbf{a}}$, and hence $\lim f \leq \mathbf{a}$. But recall that $f$ is from $U(B)$ and hence $\lim f \in e[B]$, which means that for each $i<\rho,(\lim f)(i) \in\left\{\top_{\mathbf{2} \times L}, \perp_{\mathbf{2} \times L}\right\}$. Now for each $i<\rho, \mathbf{a}(i)<\top_{2 \times L}$. Obviously then, the only element in $e[B]$ that is below a is $\perp_{(\mathbf{2} \times L)^{\rho}}$, and hence $\lim f=\perp_{(\mathbf{2} \times L)^{\rho}}$. So we are done showing that every atomic element in $U(B)$ is in the kernel of lim. To show that every atomless element in $U(B)$ is in the kernel of lim the strategy is exactly the same. If $f \in U(B)$ is atomless, then we can show similarly that $f \leq f_{\mathbf{l}}$. Then $\lim f$, being both below $\mathbf{l}$ and also inside $e[B]$, must be $\perp_{(2 \times L)^{\rho}}$. So this $f$ is also in the kernel of lim.

To show that $\lim ^{-1}\left(\perp_{(2 \times L)^{\rho}}\right) \subseteq S(U(B))$, pick an arbitrary $f \in U(B)$ such that $\lim f=\perp_{(\mathbf{2} \times L)^{\rho}}$. Then $f \wedge f_{\mathbf{a}}$ is also in $U(B)$ as $\lim \left(f \wedge f_{\mathbf{a}}\right)$ must also be $\perp_{(\mathbf{2} \times L)^{\rho}}$. For similar reasons, $f \wedge f_{\mathbf{l}} \in U(B)$ too. Now clearly $f \wedge f_{\mathbf{a}}$ is atomic in $U(B)$ since it is the join of $\left\{g_{i, j} \mid\left(f \wedge f_{\mathbf{a}}\right)(i)(j)=\left\langle\top_{\mathbf{2}}, \perp_{L}\right\rangle\right\}$ where $g_{i, j}$ is the function that always returns $\perp_{\mathbf{2} \times L}$ expect that $g_{i, j}(i)(j)=\left\langle\top_{\mathbf{2}}, \perp_{L}\right\rangle$. Each $g_{i, j}$ is obviously in $U(B)$ and is atomic. Hence $f \wedge f_{\mathbf{a}}$ is a join of atoms in $U(B)$ and hence atomic. Similarly $f \wedge f_{\mathbf{l}}$ is atomless in $U(B)$ as it is the join of the elements of the form $h_{i, j}$ below it where $h_{i, j}$ always returns $\perp_{\mathbf{2} \times L}$ except that $h_{i, j}(i)(j)=\left\langle\perp_{\mathbf{2}}, \top_{L}\right\rangle$. Each $h_{i, j}$ is in $U(B)$ and is atomless. Hence $f \wedge f_{\mathbf{l}}$ is atomless. But then, $f$ is separable by definition since $f=\left(f \wedge f_{\mathbf{a}}\right) \vee\left(f \wedge f_{\mathbf{l}}\right)$.

Now we can sum the above up and obtain the following proposition.

Proposition 4.9 For every Boolean algebra A, there is a $\left(2^{\omega_{0}}\right)^{+}$-field-of-sets $B$ which is elementarily equivalent to $A$.

Proof Let $\kappa$ be $\left(2^{\omega_{0}}\right)^{+}$. It is a successor cardinal, so it is regular. By Proposition 4.7, it is enough to show that for every $c \in \operatorname{Inv}$, there is a $\left(2^{\omega_{0}}\right)^{+}$-field-of-sets $B$ such that $\operatorname{Inv}(B)=c$. Now Inv can be partitioned into three parts: $\{\langle-1,0\rangle\},\{\langle m, n\rangle \mid m \in$ $\left.\mathbb{N}, n \in \mathbb{Z}^{\infty}\right\}$, and $\{\langle\infty, 0\rangle\}$. For $\langle-1,0\rangle$, we use $\wp(\varnothing)$. For the second part, we use a simple induction on the first coordinate.

1. For non-zero $n \in \mathbb{N}, \operatorname{Inv}(\wp(n))=\langle 0, n\rangle$, and $\operatorname{Inv}(\wp(\mathbb{N}))=\langle 0, \infty\rangle$. For $\langle 0,0\rangle$, use the $L$ from Proposition 4.8 , which is an atomless $\kappa$-field-of sets. For invariants $\langle 0,-n\rangle(n>0)$ and $\langle 0,-\infty\rangle$, use $\wp(n) \times L$ and $\wp(\mathbb{N}) \times L$, respectively.

2. Suppose for any $n \in \mathbb{Z}^{\infty}$, there is a $\kappa$-field-of-sets $B_{n}$ such that $\operatorname{Inv}\left(B_{n}\right)=$ $\langle m, n\rangle$. Then for $\langle m+1, n\rangle$ for any $n \in \mathbb{Z}^{\infty}$, use $U\left(B_{n}\right)$, since by Lemma 4.15 , $U\left(B_{n}\right)$ is a $\kappa$-field-of-sets, $U\left(B_{n}\right) / S\left(U\left(B_{n}\right)\right) \cong B_{n}$, and thus $\operatorname{Inv}\left(U\left(B_{n}\right)\right)=$ $\operatorname{Inv}\left(B_{n}\right)+\langle 1,0\rangle=\langle m+1, n\rangle$.

For the invariant $\langle\infty, 0\rangle$, take the product $B=\Pi_{i \in \mathbb{N}} U^{i}(\mathbf{2})=\mathbf{2} \times U(\mathbf{2}) \times U(U(\mathbf{2})) \times$ $U(U(U(\mathbf{2}))) \cdots$. That $B$ is a $\kappa$-field-of-sets follows from Proposition 4.4. Also, since $B / S(B)=\Pi_{i \in \mathbb{N}} U^{i}(\mathbf{2}) / S\left(U^{i}(\mathbf{2})\right)=\mathbf{1} \times \Pi_{i \in \mathbb{N}, i>0} U^{i-1}(\mathbf{2}), B / S(B)$ is isomorphic to $B$. (1 is the trivial algebra, and it appears here as the result of $2 / S(\mathbf{2})$.) This means that for any $n \in \mathbb{N}, B^{(n)}$ is isomorphic to $B$, which means that $\operatorname{Inv}(B)=\langle\infty, 0\rangle$.

The only missing link now is the following proposition, shown in [51]. 
Proposition 4.10 (Vermeer 1996) Every $\left(2^{\omega_{0}}\right)^{+}$-field-of-sets is a quotient of a complete Boolean algebra.

With this, we can prove the following lemma, which leads to a proof of Lemma 4.8 that also takes care of the requirements for the distinguished elements $z$ and $g$.

Lemma 4.16 For every Boolean algebra $A$ there is a non-trivial complete Boolean algebra $C$ with a filter $H \subseteq C$ such that $\bigwedge H=\perp$, and that $A$ is elementarily equivalent to $\mathrm{C} / \mathrm{H}$.

Proof If $A$ is trivial, let $D$ be the two-element Boolean algebra and $H$ the improper filter in $D$. Now pick an arbitrary non-trivial Boolean algebra $A$. By Proposition 4.9, there is a $\left(2^{\omega_{0}}\right)^{+}$-field-of-sets $B$ that is elementarily equivalent to $A$. Then we only need to find a complete Boolean algebra $C$ such that $C \leftrightarrow \leftrightarrow B$.

Notice that $\left(2^{\omega_{0}}\right)^{+}$is a successor cardinal, so it is regular. Then, by Proposition 4.6, there is a $\left(2^{\omega_{0}}\right)^{+}$-field-of-sets $B^{\prime}$ such that $B^{\prime} \odot \rightarrow B$. By Proposition 4.10, then, there is a complete Boolean algebra $C$ such that $C \rightarrow B^{\prime}$. But then, by Proposition 4.5, $C \odot \nrightarrow B$. Since $A$ is non-trivial, $B$ and hence $C$ must also be non-trivial.

Prooffor Lemma 4.8 Pick an arbitrary zg-algebra $\langle A, z, g\rangle$. We decompose $A$ as $\left.A\right|_{\bar{z}} \times\left. A\right|_{z g} \times\left. A\right|_{z \bar{g}}$ since $g \leq z$. Since we are only after elementary equivalence, by Proposition 4.7, it is enough to find $\left\langle A^{\prime}, z^{\prime}, g^{\prime}\right\rangle$ such that $\operatorname{Inv}\left(A^{\prime} \mid \overline{z^{\prime}}\right)=\operatorname{Inv}\left(\left.A\right|_{\bar{z}}\right)$, $\operatorname{Inv}\left(\left.A^{\prime}\right|_{z^{\prime} g^{\prime}}\right)=\operatorname{Inv}\left(\left.A\right|_{z g}\right)$, and $\operatorname{Inv}\left(\left.A^{\prime}\right|_{z^{\prime}} \overline{g^{\prime}}\right)=\operatorname{Inv}\left(\left.A\right|_{z \bar{g}}\right)$, and that $\left\langle\dot{A}^{\prime}, z^{\prime}, g^{\prime}\right\rangle$ is the natural quotient of a complete proper filter algebra.

By the definition of zg-algebra, $\left.A\right|_{z g}$ is atomic and $\left.A\right|_{z \bar{g}}$ is atomless. Let $A_{2}^{\prime}$ and $A_{3}^{\prime}$ be the MacNeille completion of $\left.A\right|_{z g}$ and $\left.A\right|_{z \bar{g}}$, respectively. Note that MacNeille completion does not change the number of atoms. Thus $\operatorname{Inv}\left(A_{2}^{\prime}\right)=\operatorname{Inv}\left(\left.A\right|_{z g}\right)$, and $\operatorname{Inv}\left(A_{3}^{\prime}\right)=\operatorname{Inv}\left(\left.A\right|_{z \bar{g}}\right)$.

To figure out $\left.A^{\prime}\right|_{\bar{z}}$, we invoke Lemma 4.16. By that lemma, there is a non-trivial complete Boolean algebra $C$ with a filter $H$, such that $C / H$ is elementarily equivalent to $\left.A\right|_{\bar{z}}$, and that $\wedge H=\perp_{C}$. Let $A_{1}^{\prime}=C / H$.

Now let $\left\langle A^{\prime}, z^{\prime}, g^{\prime}\right\rangle=\left\langle A_{1}^{\prime} \times A_{2}^{\prime} \times A_{3}^{\prime},\langle\perp, \top, \top\rangle,\langle\perp, \perp, \top\rangle\right\rangle$. Then by construction, $\left\langle A^{\prime}, z^{\prime}, g^{\prime}\right\rangle$ is also a zg-algebra and is elementarily equivalent to $\langle A, z, g\rangle$.

Then let $\mathscr{B}=\langle B, F\rangle$ where $B=C \times A_{2}^{\prime} \times A_{3}^{\prime}$ and $F=H \times\{\top\} \times\{\top\}$. For this $\mathscr{B}$, we need to establish two points.

- First, $\mathscr{B}$ is a complete proper filter algebra. To see this, note first that $C \times A_{2}^{\prime} \times A_{3}^{\prime}$ is a complete Boolean algebra as each of the three components are. Then note that $F$ is a proper filter. It is obviously a filter. It is proper because if not, $A_{1}^{\prime}$, $A_{2}^{\prime}$, and $A_{3}^{\prime}$ are all trivial, and hence $A^{\prime}$ and $A$ are trivial since $A^{\prime}$ and $A$ are elementarily equivalent. But $A$ is a zg-algebra and zg-algebras are non-trivial. Note though that any two of $A_{1}^{\prime}, A_{2}^{\prime}$, and $A_{3}^{\prime}$ can be trivial together.

- Also, the natural quotient of $\mathscr{B}, \mathscr{B} / F$, is precisely $\left\langle A^{\prime}, z^{\prime}, g^{\prime}\right\rangle$. That $A^{\prime}=B / F$ is a simple Boolean algebra exercise. The next thing to note is that $z_{\mathscr{B}}=\bigwedge F=$ $\langle\perp, \top, \top\rangle \in B$ since $\bigwedge H=\perp_{C}$. Hence $\pi_{F}\left(z_{\mathscr{B}}\right)=\langle\perp, \top, \top\rangle \in A^{\prime}$, which is precisely $z^{\prime}$. Also, the join of atoms below $z \mathscr{B}$ in $B$ is precisely $\langle\perp, \top, \perp\rangle$ as $A_{2}^{\prime}$ by construction is atomic and $A_{3}^{\prime}$ is atomless. 
The extra constraints in Lemma 4.8 are also satisfied. $\left.\mathscr{B}\right|_{z \mathscr{B}} \cong C$ is always nontrivial by construction. $\left.\mathscr{B}\right|_{z_{\mathscr{B}} g_{\mathscr{B}}} \cong A_{2}^{\prime}$ is trivial if and only if $\left.A\right|_{z g}$ is trivial since the construction method is MacNeille completion. By the same reason, $\left.\mathscr{B}\right|_{z \mathscr{B}} \overline{g_{\mathscr{B}}} \cong A_{3}^{\prime}$ is trivial if and only if $\left.A\right|_{z \bar{g}}$ is trivial.

\section{Stronger Logics and Decidability}

In the previous section, our only goal was the completeness theorems Theorem 4.1 and Theorem 4.2. However, the method we used to show them in fact supports a full analysis of the expressivity of $\mathscr{L} \Pi$ on complete proper filter algebras and the normal $\Pi$-extension of $\mathrm{KD} 4{ }^{\forall} 5 \Pi$, similar to the one in [42]. In light of the space such a general analysis would take, in this section we focus only on several natural concrete cases in which we only add one formula, or equivalently finitely many formulas, to $\mathrm{KD} 4{ }^{\forall} 5 \Pi$. Since $\mathrm{KD} 4{ }^{\forall} 5 \Pi \mathrm{zg}$ is a definitional extension of $\mathrm{KD} 4^{\forall} 5 \Pi$, in this section we move between $\mathrm{KD} 4{ }^{\forall} 5 \Pi$ and $\mathrm{KD} 4{ }^{\forall} 5 \Pi z \mathrm{z}$ freely in stating the results, noting that to obtain the results in $\mathscr{L} \Pi$, one only needs to replace $\mathrm{z}$ and $\mathrm{g}$ by their definitions in the axioms $z$ and $g$ in Definition 4.9.

Before we start, let us introduce a bit of notation. For any $X \subseteq \mathscr{L}$ Пzg, let $\operatorname{CPFA}(\Gamma)$ be the class of complete proper filter algebras validating every formula in $\Gamma$. As usual we write $\operatorname{CPFA}(\varphi)$ for $\operatorname{CPFA}(\{\varphi\})$ and write $\operatorname{CPFA}$ for $\operatorname{CPFA}(\varnothing)$, the class of all complete proper filter algebras. Then for any class $\mathrm{K}$ of complete proper filter algebras, we write $\log (\mathrm{K})$ for the set of formulas in $\mathscr{L}$ Пzg that are valid in all complete proper filter algebras in K. Finally, as usual, for any $\varphi \in \mathscr{L} \Pi z$, we write $\mathrm{KD} 4^{\forall} 5 \Pi z g \varphi$ for the smallest normal $\Pi$-logic extending $\mathrm{KD} 4^{\forall} 5 \Pi z g$ with $\varphi$. Then, we first define the following semantics-preserving mapping between complete proper filter algebras.

Definition 5.1 For any complete proper filter algebras $\mathscr{B}$ and $\mathscr{B}^{\prime}$ and any function $f$ from $\mathscr{B}$ to $\mathscr{B}^{\prime}$, we say $f$ is a complete homomorphism if

- $f$ is a complete Boolean homomorphism: $f(\neg a)=\neg f(a)$ and $f(\bigwedge X)=$ $\bigwedge f[X]$

- for any $a \in \mathscr{B}, a \in F_{\mathscr{B}}$ iff $f(a) \in F_{\mathscr{B}^{\prime}}$.

Proposition 5.1 If $f: \mathscr{B} \rightarrow \mathscr{B}^{\prime}$ is a complete homomorphism, then for any valuation $\theta$ on $\mathscr{B}$ and $\varphi \in \mathscr{L} \Pi z \mathrm{zg}, f(\tilde{\theta}(\varphi))=\widetilde{f \circ \theta}(\varphi)$.

Proof If $\varphi \in \mathscr{L} \Pi$, a simple induction suffices. For $\varphi \in \mathscr{L} \Pi z g$, note that we have the definitional axioms $\mathrm{z}$ and $\mathrm{g}$ that are sound.

Now we prove the following general completeness theorem.

Theorem 5.1 For any $\varphi \in \mathscr{L} \Pi z g, \operatorname{KD} 4^{\forall} 5 \Pi z g \varphi=\log (\operatorname{CPFA}(\varphi))$. 
Proof That $\mathrm{KD} 4{ }^{\forall} 5 \Pi z g \varphi \subseteq \log (\operatorname{CPFA}(\varphi))$ is trivial by soundness. Now pick an arbitrary $\psi \in \log (\operatorname{CPFA}(\varphi))$. Without loss of generality we assume that both $\varphi$ and $\psi$ are sentences. Since we will only be dealing with sentences whose semantic values do not depend on particular valuations, we use the notation $\mathscr{B}(\chi)$ for the semantic value of any sentence $\chi$ in $\mathscr{B}$. By necessitation and modus ponens in $\mathrm{KD} 4{ }^{\forall} 5 \Pi \mathrm{zg}$, it is enough to show that $\vdash(\varphi \wedge \mathrm{B} \varphi) \rightarrow \psi$. By the completeness theorem, then, it is enough to show that for every $\mathscr{B} \in \mathrm{CPFA}, \mathscr{B} \vDash(\varphi \wedge \mathrm{B} \varphi) \rightarrow \psi$.

Pick an arbitrary complete proper filter algebra $\mathscr{B}$. If $\mathscr{B}(\varphi) \notin F_{\mathscr{B}}$, then we are done since $\mathscr{B}(B \varphi)$ and hence $\mathscr{B}(\varphi \wedge B \varphi)$ in this case is $\perp$. So now we focus on the case where $\mathscr{B}(\varphi) \in F_{\mathscr{B}}$ and let $v=\mathscr{B}(\varphi)$. Consider $\mathscr{B}^{\prime}$ defined by restricting $\mathscr{B}$ to $v: \mathscr{B}^{\prime}=\left\langle\mathscr{B} \mid v,\left\{a \wedge v \mid a \in F_{\mathscr{B}}\right\}\right\rangle$. It is not hard to see that $h: \mathscr{B} \rightarrow \mathscr{B}^{\prime}$ defined by $h(a)=a \wedge v$ is a complete homomorphism. By Proposition 5.1, we see that $(1) \mathscr{B}^{\prime}(\varphi)=h(\mathscr{B}(\varphi))=v=\top_{\mathscr{B}}$ and also that $(2) \mathscr{B}^{\prime}(\psi)=h(\mathscr{B}(\psi))$. From (1), it follows that $\mathscr{B}^{\prime} \vDash \varphi$. By assumption, $\mathscr{B}^{\prime} \vDash \psi$. Hence, by $(2), h(\mathscr{B}(\psi))=$ $\mathscr{B}(\psi) \wedge v=v$ and thus $\mathscr{B}(\psi) \geq v$. This means that $\mathscr{B}(\psi) \geq \mathscr{B}(\varphi \wedge B \varphi)$ and that $\mathscr{B} \vDash(\varphi \wedge B \varphi) \rightarrow \psi$.

While of course there is a limit to the expressivity of $\mathscr{L} \Pi z \mathrm{z}$, many natural classes of complete proper filter algebras corresponds to the validity of sentences in $\mathscr{L}$ Пzg. We give some examples below.

Corollary 5.1 - $\operatorname{CPFA}(\mathrm{z})$ is the class of complete proper filter algebras with trivial filters. Hence its logic is $\mathrm{KD} 4{ }^{\forall} 5 \Pi \mathrm{zgz}$. In $\mathscr{L} \Pi$, the logic is $\mathrm{KD} 4{ }^{\forall} 5 \Pi \forall p(\mathrm{~B} p \rightarrow p)$.

- $\quad \mathrm{CPFA}(\mathrm{Bz})$ is the class of complete proper filter algebras with principal filters. Hence its logic is $\mathrm{KD} 4{ }^{\forall} 5 \Pi \mathrm{zgBz}$. In $\mathscr{L} \Pi$, the logic is $\mathrm{KD} 4{ }^{\forall} 5 \Pi$ Immod.

- The class of complete proper filter algebras with ultrafilters is defined by $\forall p(\mathrm{~B} p \vee \mathrm{B} \neg p)$. Hence its logic in $\mathscr{L} \Pi$ is $\mathrm{KD} 4{ }^{\forall} 5 \Pi \forall p(\mathrm{~B} p \vee \mathrm{B} \neg p)$.

Now we consider an undefinable property: atomicity. To see that it is not definable by the validity of any formula in $\mathscr{L} \Pi z \mathrm{z}$, we first establish a general proposition.

Proposition 5.2 For any complete proper filter algebras $\mathscr{B}$ and $\mathscr{B}^{\prime}$, if $\overline{z_{\mathscr{B}}}$ and $\overline{z_{\mathscr{B}^{\prime}}}$ are non-trivial and $\mathscr{B} / F_{\mathscr{B}}$ and $\mathscr{B}^{\prime} / F_{\mathscr{B}}$ are elementarily equivalent, then $\log (\mathscr{B})=$ $\log \left(\mathscr{B}^{\prime}\right)$.

Proof Let $\mathscr{B}$ and $\mathscr{B}^{\prime}$ with the suppositions above be given. Observe first that when passing from $\mathscr{B}$ to $\mathscr{B} / F_{\mathscr{B}}$, the Boolean structure of $\mathscr{B}$ below $z_{\mathscr{B}}$ and the Boolean structure of $\mathscr{B} / F_{\mathscr{B}}$ below $z_{\mathscr{B}} / F_{\mathscr{B}}$ are the same. In fact, if $B$ is a complete Boolean algebra, $F$ a filter in $B$, and $z$ the meet of $F$, then $B / F$ is isomorphic to $\left(\left.B\right|_{\bar{z}} /\left.F\right|_{\bar{z}}\right) \times$ $\left.B\right|_{z}$ where $\left.F\right|_{\bar{z}}=\{a \wedge \neg z \mid a \in F\}$. Thus, the $z g$ in $\mathscr{B}$ is trivial iff the $z g$ in $\mathscr{B} / F_{\mathscr{B}}$ is trivial, and the same goes for $z \bar{g}$ and for $\mathscr{B}^{\prime}$. Since $\mathscr{B} / F_{\mathscr{B}}$ and $\mathscr{B}^{\prime} / F_{\mathscr{B}^{\prime}}$ are elementarily equivalent, $z g$ (resp. $z \bar{g}$ ) in $\mathscr{B}$ is non-trivial iff $z g$ (resp. $z \bar{g}$ ) in $\mathscr{B}^{\prime}$ is non-trivial. Since we also assumed that the $\bar{z}$ in both $\mathscr{B}$ and $\mathscr{B}^{\prime}$ are non-trivial, in sum, the triviality of $\bar{z}, z g$, and $z \bar{g}$ in $\mathscr{B}$ and $\mathscr{B}^{\prime}$ are the same, respectively. 
Now, recall that by Lemma 4.7, for any sentence $\varphi \in \mathscr{L}$ Пzg, we can assume that $\varphi=\left(\overline{\mathrm{z}} \wedge \varphi_{\overline{\mathrm{z}}}\right) \vee\left(\mathrm{zg} \wedge \varphi_{\mathrm{zg}}\right) \vee\left(\mathrm{z} \overline{\mathrm{g}} \wedge \varphi_{\mathrm{z} \overline{\mathrm{g}}}\right)$ where $\varphi_{\overline{\mathrm{z}}}, \varphi_{\mathrm{zg}}$, and $\varphi_{\mathrm{z} \overline{\mathrm{g}}}$ are all in $\mathscr{L} \mathrm{B} \Pi z \mathrm{z}$. This means, given Lemma 4.1 and that the natural quotients of $\mathscr{B}$ and $\mathscr{B}^{\prime}$ are elementarily equivalent, $\mathscr{B} \vDash \chi$ iff $\mathscr{B}^{\prime} \vDash \chi$ for all $\chi \in\left\{\varphi_{\overline{\mathrm{z}}}, \varphi_{\mathrm{zg}}, \varphi_{\mathrm{z} \overline{\mathrm{g}}}\right\}$. By the simple reasoning we have used in the beginning of the proof of Theorem $4.2, \mathscr{B} \vDash \varphi$ iff $\mathscr{B}^{\prime} \vDash \varphi$. To show the same for formulas with free variables, take the universal closure of them.

Proposition 5.3 There are no $\Gamma \subseteq \mathscr{L}$ Пzg such that CPFA $(\Gamma)$ is precisely the class of atomic complete proper filter algebras.

Proof Let $\mathscr{B}_{0}=\left\langle\wp(\mathbb{N}), F_{0}\right\rangle$ where $F_{0}$ is a non-principal ultrafilter of $\wp(\mathbb{N})$. Then let $\mathscr{B}_{1}=\left\langle L, F_{1}\right\rangle$ where $L$ is a complete atomless Boolean algebra and $F_{1}$ is an ultrafilter in $L$. Note that for both $i \in\{0,1\}, z_{\mathscr{B}_{i}}$ is $\perp$ and the natural quotient $\mathscr{B}_{i} / F_{i}$ is isomorphic to $\langle\mathbf{2}, \perp, \perp\rangle$ where $\mathbf{2}$ is a two-element Boolean algebra. By Proposition 5.2, for any $\varphi \in \mathscr{L} \Pi z g, \mathscr{B}_{0} \vDash \varphi$ if and only if $\mathscr{B}_{1} \vDash \varphi$. But $\mathscr{B}_{0}$ is atomic yet $\mathscr{B}_{1}$ is not.

However, the undefinability of atomicity in complete proper filter algebras does not preclude axiomatization. An obvious validity on atomic complete proper filter algebras is $z \rightarrow g$ since $z$ must be below the join of atoms below $z$. It turns out that we can just append this to $\mathrm{KD} 4{ }^{\forall} 5 \Pi \mathrm{zg} \varphi$ to obtain the logic of the atomic algebras in $\operatorname{CPFA}(\varphi)$. To show this, first note that we can strengthen Lemma 4.16 so that the nontrivial complete Boolean algebra $C$ is also atomic. This can be done simply by using the canonical extension $C^{\delta}$, the powerset algebra of the set of ultrafilters of $C$, rather than $C$ as the final result of that lemma, since by Sikorski's extension lemma and $C$ being complete, $C^{\delta} \rightarrow C$ (for a proof, see [53], Theorem 5, Chapter 13). We can then chain the surjective morphisms and see that $C^{\delta} \leftrightarrow B$ where $B$ is elementarily equivalent to an arbitrarily given Boolean algebra. But then, the Lemma 4.8 is also strengthened so that besides all other requirements, $\overline{z_{\mathscr{B}}}$ can be atomic. In sum, the completeness theorem is now strengthened into the following: if $\varphi \in \mathscr{L}$ חzg is valid on all complete proper filter algebras $\mathscr{B}$ such that $\overline{z_{\mathscr{B}}}$ is atomic, then $\varphi$ is already in $\mathrm{KD} 4{ }^{\forall} 5 \Pi \mathrm{zg}$. To formulate results below, let us use CPFA ${ }^{\bar{z} a t}(\Gamma)$ to denote the class of complete proper filter algebras such that $\bar{z}$ is atomic and every formula in $\Gamma$ is validated and use $\operatorname{CPFA}^{\text {at }}(\Gamma)$ for the class of complete proper filter algebras that are atomic and validates everything in $\Gamma$.

Theorem 5.2 For every formula $\varphi \in \mathscr{L} \Pi z g, \operatorname{KD} 4^{\forall} 5 \Pi z g \varphi=\log \left(\operatorname{CPFA}^{\bar{z} a t}(\varphi)\right)$. For $\mathrm{CPFA}^{a t}$, we have that $\mathrm{KD}^{\forall} 5 \Pi z \mathrm{z}((\mathrm{z} \rightarrow \mathrm{g}) \wedge \varphi)=\log \left(\mathrm{CPFA}^{a t}(\varphi)\right)$.

Proof To show that $\mathrm{KD} 4^{\forall} 5 \Pi z g \varphi=\log \left(\operatorname{CPFA}^{\bar{z} \text { at }}(\varphi)\right)$, using Theorem 5.1, we only need to show that $\log \left(\operatorname{CPFA}^{\bar{z} \text { at }}(\varphi)\right) \subseteq \log (\operatorname{CPFA}(\varphi))$. This clearly follows from the fact that for every $\mathscr{B} \in \mathrm{CPFA}$ there is a $\mathscr{B}^{\prime} \in \mathrm{CPFA}^{\bar{z} \text { at }} \operatorname{such}$ that $\log (\mathscr{B})=\log \left(\mathscr{B}^{\prime}\right)$. If $\mathscr{B}$ is such that $\bar{z}_{\mathscr{B}}$ is trivial, then $\mathscr{B}$ itself is in CPFA $\bar{z}$ at and we are done. If $\bar{z}_{\mathscr{B}}$ is not trivial, then apply the strengthend Lemma 4.8 to $\mathscr{B} / F_{\mathscr{B}}$ and obtain $\mathscr{B}^{\prime}$. By the strengthening, $\mathscr{B}^{\prime} \in \mathrm{CPFA}^{\bar{z} \text { at }}$. Moreover, Lemma 4.8 states that the $z g$ (resp. $z \bar{g}$ ) in $\mathscr{B}^{\prime}$ is non-trivial iff the $z g$ (resp. $z \bar{g}$ ) in $\mathscr{B} / F_{\mathscr{B}}$ is non-trivial. This means that 
Proposition 5.2 can be applied to $\mathscr{B}$ and $\mathscr{B}^{\prime}$, and from it we have that $\log (\mathscr{B})=$ $\log \left(\mathscr{B}^{\prime}\right)$.

To show that $\operatorname{KD} 4{ }^{\forall} 5 \Pi z g((z \rightarrow g) \wedge \varphi)=\log \left(\operatorname{CPFA}^{\text {at }}(\varphi)\right)$, note that $\mathrm{CPFA}^{\bar{z} \text { at }}(\mathrm{z} \rightarrow \mathrm{g})=\mathrm{CPFA}^{\text {at }}$ and thus $\operatorname{CPFA}^{\text {at }}(\varphi)=\mathrm{CPFA}^{\bar{z} \text { at }}((\mathrm{z} \rightarrow \mathrm{g}) \wedge \varphi)$, since for any $\mathscr{B} \in \mathrm{CPFA}, z_{\mathscr{B}} g_{\mathscr{B}}$ is already atomic.

With the above general completeness, we have the following corollary collecting a few special cases. To state results in language $\mathscr{L} \Pi$, let At stand for $\forall p(\mathrm{~B} p \rightarrow$ $p) \rightarrow \exists p(p \wedge a t(p))$ where $a t(p)$ is defined as in Definition 4.4 except that all $z$ used in $a t(p)$ is replaced by $\forall p(\mathrm{~B} p \rightarrow p)$.

Corollary 5.2 - The logic of the class of atomic complete proper filter algebras with trivial filters in language $\mathscr{L} \Pi$ is $\mathrm{KD} 4{ }^{\forall} 5 \Pi \mathrm{A} \forall \forall p(\mathrm{~B} p \rightarrow p)$.

- The logic of the class of atomic complete proper filter algebras with principal filters in language $\mathscr{L} \Pi$ is $\mathrm{KD} 4{ }^{\forall} 5$ ПAt Immod.

- The logic of the class of atomic complete proper filter algebras with ultrafilters in language $\mathscr{L} \Pi$ is $\mathrm{KD} 4{ }^{\forall} 5 \Pi \mathrm{A} t \forall p(\mathrm{~B} p \vee \mathrm{B} \neg p)$.

Before we move on to decidability, note that since $\mathrm{KD} 4{ }^{\forall} 5 \Pi$ is sound on the class of all complete KD45 algebras, the above general completeness theorems, when phrased in $\mathscr{L} \Pi$ (since we didn't define the semantics of $\mathrm{z}$ and $\mathrm{g}$ on those algebras), hold for complete KD45 algebras too.

For decidability, the situation is simple: all the logics mentioned above are decidable. To see this, we first state a general theorem linking the decidability of logics in the form of $\log (K)$ to the decidability of the first-order theories of some classes of $\mathrm{zg}$-algebras coming from $\mathrm{K}$.

Theorem 5.3 For any $\mathrm{K} \subseteq \mathrm{CPFA}$, Let $Q \mathrm{~K}_{0}=\left\{\mathscr{B} / F_{\mathscr{B}} \mid \mathscr{B} \in \mathrm{K}\right.$ and $\left.\overline{z_{\mathscr{B}}}=\perp\right\}$ and $Q \mathrm{~K}_{1}=\left\{\mathscr{B} / F_{\mathscr{B}} \mid \mathscr{B} \in \mathrm{K}\right.$ and $\left.\overline{z_{\mathscr{B}}} \neq \perp\right\}$. Then, if $Q \mathrm{~K}_{0}$ and $Q \mathrm{~K}_{1}$ have a decidable first-order theory in $\mathscr{F} \mathscr{O L} \mathrm{zg}$, then $\mathrm{Log}(\mathrm{K})$ is decidable.

Proof For any $\varphi \in \mathscr{L}$ Пzg, to decide whether $\varphi \in \log (\mathrm{K})$, we can first take its universal closure and then turn it into a sentence of the form

$$
(\overline{\mathrm{z}} \wedge \alpha) \vee(\mathrm{zg} \wedge \beta) \vee(\mathrm{z} \overline{\mathrm{g}} \wedge \gamma)
$$

with $\alpha, \beta, \gamma \in \mathscr{L} \mathrm{B}$ Izg. Obviously this process is decidable. Then, following similar reasoning done in the proof of Theorem $4.2, \varphi \in \log (\mathrm{K})$ if and only if for all $\mathscr{B} \in \mathrm{K}$, the following hold.

- Either $z_{\mathscr{B}} g_{\mathscr{B}}=\perp$ or $\mathscr{B} / F_{\mathscr{B}} \vDash \mathscr{T}(\beta)$.

- Either $z \mathscr{B} \overline{g_{\mathscr{B}}}=\perp$ or $\mathscr{B} / F_{\mathscr{B}} \vDash \mathscr{T}(\gamma)$.

- Either $\overline{z_{\mathscr{B}}}=\perp$ or $\mathscr{B} / F_{\mathscr{B}} \vDash \mathscr{T}(\alpha)$.

It is not hard to see that $z_{\mathscr{B}} g_{\mathscr{B}}=\perp$ if and only if $\mathscr{B} / F_{\mathscr{B}} \vDash((\mathrm{z} \wedge \mathrm{g})=\perp)$, and similarly $z_{\mathscr{B}} \overline{g_{\mathscr{B}}}=\perp$ if and only if $\mathscr{B} / F_{\mathscr{B}} \vDash((\mathrm{z} \wedge \neg \mathrm{g})=\perp)$. This is because $z_{\mathscr{B}}=\bigwedge F_{\mathscr{B}}$, so all distinctions below $z_{\mathscr{B}}$ are preserved under quotienting through $F_{\mathscr{B}}$. However, there is no analog for $\overline{z_{\mathscr{B}}}$. It may well be that $\mathscr{B} / F_{\mathscr{B}} \vDash \neg \mathrm{Z}=\perp$ while 
$\overline{z_{\mathscr{B}}}>\perp$. This happens whenever $F_{\mathscr{B}}$ is principal, and this is why we need to take care of two classes of natural quotients. Using the observations we collected, now $\varphi \in \log (\mathrm{K})$ if and only if the following hold.

- For all $\mathscr{B} \in \mathrm{K}$ such that $\overline{z_{\mathscr{B}}}=\perp, \mathscr{B} / F_{\mathscr{B}} \vDash((\mathrm{zg}=\perp) \vee \mathscr{T}(\beta)) \wedge((\mathrm{z} \overline{\mathrm{g}}=$ $\perp) \vee \mathscr{T}(\gamma))$.

- For all $\mathscr{B} \in \mathrm{K}$ such that $\overline{z \mathscr{B}} \neq \perp, \mathscr{B} / F_{\mathscr{B}} \vDash \mathscr{T}(\alpha) \wedge((\mathrm{zg}=\perp) \vee \mathscr{T}(\beta)) \wedge((\mathrm{z} \overline{\mathrm{g}}=$ $\perp) \vee \mathscr{T}(\gamma))$.

Thus we are now deciding if two formulas, obtained effectively from $\varphi$, are in the first-order theories of $Q \mathrm{~K}_{0}$ and $Q \mathrm{~K}_{1}$ respectively. By assumption the two theories are decidable. Hence whether $\varphi \in \log (\mathrm{K})$ is decidable.

Theorem 5.4 The following logics are decidable:

- $\mathrm{KD} 44^{\forall} 5$,

- $\mathrm{KD} 4^{\forall} 5 \Pi \forall p(\mathrm{~B} p \rightarrow p)$,

- $\mathrm{KD} 4{ }^{\forall} 5 \Pi \mathrm{B} \forall p(\mathrm{~B} p \rightarrow p)$,

- $\mathrm{KD} 4^{\forall} 5 \Pi \forall p(\mathrm{~B} p \vee \mathrm{B} \neg p)$,
$-\mathrm{KD} 4^{\forall} 5$ П $\mathrm{At}$,

$-\mathrm{KD} 4^{\forall} 5 \Pi$ П $\forall p(\mathrm{~B} p \rightarrow p)$,

$-\mathrm{KD} 4{ }^{\forall} 5$ ПАt $\mathrm{B} \forall p(\mathrm{~B} p \rightarrow p)$,

$-\mathrm{KD} 4{ }^{\forall} 5 \Pi A t \forall p(\mathrm{~B} p \vee \mathrm{B} \neg p)$.

Proof As is argued above, each of them comes from a well-behaved class of complete proper filter algebras. Take $\mathrm{KD} 4^{\forall} 5 \Pi$ for example. It is the logic of CPFA. Using the notation above in Theorem 5.3, we only need to argue that $Q$ CPFA ${ }_{0}$ and $Q \mathrm{CPFA}_{1}$ have decidable theories in $\mathscr{F} \mathscr{O L} \mathbf{z g}$.

- $Q \mathrm{CPFA}_{0}$ is just the class of complete zg-algebras with $z$ being the top element. This is because that if $\mathscr{B}=\langle B, F\rangle$ is such that $\overline{z_{\mathscr{B}}}=\perp$, then $z_{\mathscr{B}}=\mathrm{T}$, and hence $F$ is the trivial filter. Thus $\mathscr{B} \mid F=\langle B, \top, g\rangle$ where $g$ is the join of atoms. It is well known that the first-order theory of non-trivial complete Boolean algebras is decidable. To decide whether $\varphi \in \mathscr{F} \mathscr{O L}$ zg is valid in $Q \mathrm{CPFA}_{0}$, we only need to test whether the formula $(x=\top \wedge a t(y)) \rightarrow \varphi[x / z, y / \mathrm{g}]$ in $\mathscr{F} \mathscr{O L}$ is valid in all non-trival complete Boolean algebras, where $x$ and $y$ are two fresh variables and at $(y)$ states that $y$ is the join of all atoms (which is expressible in $\mathscr{F} \mathscr{O L}$ ).

- By Lemma 4.8, we see that the theory of $Q \mathrm{CPFA}$ is precisely the theory of all zg-algebras: FOLzg. This theory is decidable since the theory of all non-trivial Boolean algebras is well kown to be decidable, and to test whether $\varphi \in \mathscr{F} \mathscr{O} \mathscr{L}$ zg is in FOLzg, we only need to test whether the formula at $(x, y) \rightarrow \varphi[x / z, y / g]$ is valid in all non-trivial Boolean algebras, where $x$ and $y$ are fresh variables and at $(x, y)$ states that $y$ is the join of the atoms below $x$.

The argument above clearly generalizes to all other cases, noting also that the firstorder theory of atomic Boolean algebras, the first-order theory of complete and atomic Boolean algebras, and the first-order theory of two-element Boolean algebras are all decidable. We briefly sketch the $\mathscr{F} \mathscr{O L}$ zg theories we need for the other logics.

- $\mathrm{KD} 4{ }^{\forall} 5 \Pi \forall p(\mathrm{~B} p \rightarrow p)$ is the logic of complete proper filter algebras with the trivial filter. Calling this class $\mathrm{K}$, the theory of $Q \mathrm{~K}_{0}$ is the theory of complete $\mathrm{zg}$ algebras with $z$ being $\mathrm{T}$, and the theory of $Q \mathrm{~K}_{1}$ is the inconsistent theory since $Q \mathrm{~K}_{1}$ is empty. 
- $\mathrm{KD} 4{ }^{\forall} 5 \Pi \mathrm{B} \forall p(\mathrm{~B} p \rightarrow p)$ is the logic of complete proper filter algebras with a principal filter. Calling this class $\mathrm{K}$, the theory of $Q \mathrm{~K}_{0}$ is the theory of complete $\mathrm{zg}$-algebras with $z$ being $\top$, and the theory of $Q \mathrm{~K}_{1}$ is also the theory of complete zg-algebras with $z$ being $T$.

- $\mathrm{KD} 4{ }^{\forall} 5 \Pi \mathrm{B} \forall p(\mathrm{~B} p \vee \mathrm{B} \neg p)$ is the logic of complete proper filter algebras with an ultrafilter. Calling this class $\mathrm{K}$, the theory of $Q \mathrm{~K}_{0}$ is the theory of two-element zg-algebras with $z$ being $\top$, and the theory of $Q \mathrm{~K}_{1}$ is the theory of two-element zg-algebras.

- For $\mathrm{KD} 4{ }^{\forall} 5 \Pi$ At, the relevant $\mathscr{F} \mathscr{O} \mathscr{L}$ zg-theories are the theory of atomic and complete zg-algebras with $z$ being $\top$ and the theory of $z g$-algebras with $g$ being equal to $z$.

- For $\mathrm{KD} 4^{\forall} 5 \Pi$ At $\forall p(\mathrm{~B} p \rightarrow p)$, the relevant $\mathscr{F} \mathscr{O} \mathscr{L}$ zg-theories are the theory of atomic and complete zg-algebras and the inconsistent theory.

- For $\mathrm{KD} 4^{\forall} 5 \Pi \mathrm{A} t \mathrm{~B} \forall p(\mathrm{~B} p \rightarrow p)$, the relevant $\mathscr{F} \mathscr{O L}$ zg-theory is the theory of atomic and complete zg-algebras with $z$ being $T$.

- For $\mathrm{KD} 4^{\forall} 5 \Pi$ At $\forall p(\mathrm{~B} p \vee \mathrm{B} \neg p)$, the relevant $\mathscr{F} \mathscr{O L} \mathscr{L g}$-theories are the theory of two-element zg-algebras with $z$ being $\top$ and the theory of two-element zgalgebras.

\section{Conclusion}

In the previous sections, we have studied complete KD45 algebras, complete proper filter algebras, and logics in $\mathscr{L} \Pi$ extending $\mathrm{KD} 45$ based on these algebras. It turns out that $\mathrm{KD} 4{ }^{\forall} 5 \Pi$ is the weakest logic we can have if we use algebraic semantics based on complete Boolean algebras of propositions to extend $\mathrm{KD} 45$ with propositional quantifiers. Beyond $\mathrm{KD} 4{ }^{\forall} 5 \Pi$, the semantics based on complete proper filter algebras is adequate for many logics, and we can even show some general completeness theorems. Moreover, the semantics is arguably intuitive for the language $\mathscr{L} \Pi$ as many properties of the algebras can be easily defined by the language, with atomicity being an exception, and we can determine decidability easily in many cases if the logic is coming from a class of complete proper filter algebras.

To conclude, we mention some directions of future research. First, noting that the set of measure 1 set in any probability space is always a proper filter in the algebra of events and that probability spaces are commonly used to model subjective credences, we may consider interpreting $\mathscr{L} \Pi$ on probability spaces and obtain a logic of "credence 1". The first difficulty for this is that in a probability space $\langle X, B, \mu\rangle$ with $B$ the algebra of events, in most realistc cases, $B$ is not lattice complete. To overcome this, it would be good to pin down exactly what is required for the well-definedness of the semantics of $\mathscr{L} \Pi$ and see how widely applicable the requirement is. Once this is done, to obtain the logic, our strategy above suggests that we need to study the natural quotient of $\langle B, F\rangle$ by $F$, the filter of measure 1 sets. It is well known that if $B$ is a $\sigma$-algebra and $\mu$ is countably additive, then $B / F$ is lattice complete. Roughly speaking, then, the first-order theory of the natural quotients of countably additive probability spaces by their filter of measure 1 sets is at least the first-order theory of 
complete Boolean algebras. On the other hand, if we do not assume countable additivity, then there seems to be little constraint on what the quotient could be. These two observations suggest that $\mathscr{L} \Pi$ is able to distinguish countably additive probability spaces from merely finitely additive probability spaces.

Second, we can include more modal operators in the language, each of which is interpreted by a proper filter. This is of course not the most general way to extend our language with multiple modalities. But if not careful, we may suddenly find ourselves on the other side of the axiomatizability boundary. Also, some special cases of this semantics may be of conceptual significance. For example, there can be two modal operators, one for "necessarily", which is interpreted using the trivial filter containing only the top element, and the other for "actually", interpreted by a complete ultrafilter, which is necessarily generated by an atom, or just an ultrafilter, if one would like to drop the assumption that there is an "actual world". Without the modality for necessity, the logic would be extending $\mathrm{KD} 4{ }^{\forall} 5 \Pi$ with both Immod and $\forall p(\mathrm{~B} p \vee \neg \mathrm{B} p)$ or just $\forall p(\mathrm{~B} p \vee \neg \mathrm{B} p)$, depending on whether the ultrafilter is principal or not, as we have shown above. Another example is when the modal operators are belief operators of different agents, where the beliefs of all agents are publicly known to all agents, so that one agent believes that $p$ if and only if any other agent believes that the former agent believes that $p$. We conjecture that the general idea of relativization to $\bar{z}, z g$, and $z \bar{g}$ on both the logic and the algebra sides can be generalized to deal with multiple filters too.

Third, recall that algebraic semantics based on complete algebras cannot distinguish $\mathrm{KD} 4{ }^{\forall} 5 \Pi$ from $\mathrm{KD} 45 \Pi$. In Section 2, we introduced semantics for $\mathscr{L} \Pi$ based on frames with propositional contingency. While it is true that to separate logics above $\mathrm{KD} 45$ using this semantics, we need drop the usual first-order correspondences of KD45 such as shift-reflexivity, this is not an insurmountable difficulty. We believe that indeed $4^{\forall}$ is not already in $\mathrm{KD} 45 \Pi$, and this can be shown by using semantics with propositional contingency. But a full proof of this shall be left for an other occasion. It is also hopeful that we can have a semantics based on propositional contingency that is adequate for $\mathrm{KD} 45 \Pi$.

Let us now consider the general method of extending normal modal logics with propositional quantifiers through complete algebras and raise some natural questions here. Let $\mathscr{L}$ be the quantifier free fragment $\mathscr{L} \Pi$, and let $\operatorname{CAlg}(\Gamma)$ be the class of lattice complete BAOs validating all formulas in $\Gamma$, with $\mathrm{CAlg}(\varphi)$ and CAlg abbreviating $\operatorname{CAlg}(\{\varphi\})$ and $\operatorname{CAlg}(\varnothing)$, respectively. Then, let $\log (\mathrm{K})$ be the set of sentences in $\mathscr{L} \Pi$ validated by every member of K. Once these two operators are defined, a series of standard questions can be asked. Most notably is the question of characterizing the fixed points of this Galois connection, namely the classes of algebras of the form $\operatorname{CAlg}(\Gamma)$ and the sets of sentences of the form $\log (\mathrm{K})$. But from the perspective of extending normal modal logics with propositional quantifiers, the natural object of study is Log o CAlg, an operator from $\wp(\mathscr{L})$ to $\wp(\mathscr{L} \Pi)$. A theorem we have shown in this paper is that $\log (\mathrm{CAlg}(\mathrm{KD} 45))=\mathrm{KD} 4{ }^{\forall} 5 \Pi$, where we see that axiom 4 is strengthened into $4^{\forall}$ (we assume that $4^{\forall}$ is not in $\mathrm{KD} 45 \Pi$ below). Note, however, that $\log (\mathrm{CAlg}(\mathrm{S} 5))=\mathrm{S} 5 \Pi$, in which case there is no strengthening of the axioms in $\mathrm{S} 5$. In other words, for $\mathrm{S} 5$, the syntactic way of extending it with propositional quantifiers by adding $\Pi$-principles and the semantic way of extending it by going through 
complete BAOs result in the same logic, while for $\mathrm{KD} 45$ this is not so. In general, let us call a normal modal logic $\mathrm{L}$ in $\mathscr{L} \mathscr{C} \Pi$-complete if $\log (\mathrm{CAlg}(\mathrm{L}))=\mathrm{L} \Pi$; that is, the syntactic way and the semantic way of extending $L$ to a $\Pi$-logic in $\mathscr{L} \Pi$ are the same judging from the final result. Then, we can ask what accounts for the distinction that KD45 is $\mathscr{C} \Pi$-incomplete yet S5 is $\mathscr{C} \Pi$-complete, and more generally we can ask whether there is a more logical or intrinsic way to characterize $\mathscr{C} \Pi$-(in)completeness.

The name " $\mathscr{C} \Pi$-completeness" we chose for the property is inspired by the wellstudied property of $\mathscr{C}$-completeness of normal modal logics in $\mathscr{L}$. Recall that by definition, using our notation, a normal modal logic $\mathrm{L} \subseteq \mathscr{L}$ is $\mathscr{C}$-complete if and only if $\log (\mathrm{CAlg}(\mathrm{L})) \cap \mathscr{L}=\mathrm{L}$. Given that the definitions of these two properties are similar in form, one might hope that there are some logical relations between them. However, if $4^{\forall}$ is not in $\mathrm{KD} 45 \Pi$, as we believe, then $\mathscr{C}$-completeness does not imply $\mathscr{C} \Pi$-completeness since $\mathrm{KD} 45$ is well known to be $\mathscr{C}$-complete (in fact Kripke complete or more algebraically $\mathscr{C} \mathscr{A} \mathscr{V}$-complete). The other direction is also not obvious. Suppose $\mathrm{L}$ is $\mathscr{C}$-incomplete. Then there is a $\varphi \in(\log (\mathrm{CAlg}(\mathrm{L})) \cap \mathscr{L}) \backslash \mathrm{L}$. If we can show that $\varphi \notin \mathrm{L} \Pi$ then we will be done. However, this is not obvious as while $\varphi \in \mathscr{L}$, it may well be that $\mathrm{L} \Pi$ is not conservative over $\mathrm{L}$ and $\mathrm{L} \Pi \cap \mathscr{L} \supsetneq \mathrm{L}$ with $\varphi$ witnessing the inequality. In general, we can call a normal modal logic $\mathrm{L}$ in $\mathscr{L} \Pi$-conservative if $\mathrm{L} \Pi \cap \mathscr{L}=\mathrm{L}$. Then, it is easy to observe that $\mathscr{C}$-incompleteness plus $\Pi$-conservativity imply $\mathscr{C} \Pi$-incompleteness. However, it seems unlikely that $\mathscr{C}$ incompleteness and $\Pi$-conservativity can coexist, since the $\Pi$-principles intuitively should help derive validities in complete BAOs that normal modal logics cannot. At any rate, the logical relations among the above three properties about normal modal logics regarding how they can be extended (with or without propositional quantifiers) using complete BAOs seem intricate and may be worthy of future research.

Finally, we would like to point out that our proof of the completeness theorem relies heavily on a syntactic reduction that can hardly be generalized below KD 45 since once we introduced $z$ and $g$, by the end of the process, we see that all quantifiers are outside the scope of the modal operators, and moreover there is only one layer of modal operators. Once we let go of the 4 and 5 axioms, we can hardly achieve this result. Our strategy may still work when we study Log(CAlg(K45)), but a method more generalizable is clearly needed if we want to venture further.

\section{References}

1. Belardinelli, F., Van Der Hoek, W., Kuijer, L.B. (2018). Second-order propositional modal logic: Expressiveness and completeness results. Artificial Intelligence, 263, 3-45.

2. Hintikka, J. (1962). Knowledge and belief: an introduction to the logic of the two notions.

3. Yap, A. (2014). Idealization, epistemic logic, and epistemology. Synthese, 191(14), 3351-3366.

4. Caie, M. (2019). Doxastic logic. In Weisberg, J., \& Pettigrew, R. (Eds.) The Open Handbook of Formal Epistemology (pp. 499-541): PhilPapers Foundation.

5. Slaney, J. (1996). KD45 is not a doxastic logic. Technical Report, TR-SRS-3-96, Australian National University.

6. Baltag, A., Bezhanishvili, N., Özgün, A., Smets, S. (2019). A topological approach to full belief. Journal of Philosophical Logic, 48(2), 205-244. 
7. Baltag, A., Bezhanishvili, N., Özgün, A., Smets, S. (2017). The topology of full and weak belief. In Hansen, H.H., Murray, S.E., Sadrzadeh, M., Zeevat, H. (Eds.) Logic, Language, and Computation (pp. 205-228). Berlin: Springer.

8. Lewis, D. (1986). On the plurality of worlds Vol. 322: Oxford Blackwell.

9. Blackburn, P., De Rijke, M., Venema, Y. (2002). Modal logic: graph Vol. 53. darst: Cambridge University Press.

10. Segerberg, K. (1971). An essay in classical modal logic.

11. van Benthem, J., \& Smets, S. van Ditmarsch, H., Halpern, J.Y., van der Hoek, W., Kooi, B. (Eds.) (2015). Dynamic logics of belief change: College Publications.

12. Holliday, W.H., \& Litak, T. (2019). Complete additivity and modal incompleteness. The Review of Symbolic Logic, 12(3), 487-535.

13. Clarke, R. (2013). Belief is credence one (in context). Philosopher's Imprint 13(11).

14. Tokarz, M. (1990). On the logic of conscious belief. Studia Logica, 49(3), 321-332.

15. Fine, K. (1969). For some proposition and so many possible worlds. Ph.D. Thesis, University of Warwick. http://wrap.warwick.ac.uk/72219/.

16. Kripke, S.A. (1959). A completeness theorem in modal logic. The journal of symbolic logic, 24(01), $1-14$.

17. Bull, R.A. (1969). On modal logic with propositional quantifiers. The Journal of Symbolic Logic, 34(2), 257-263.

18. Gabbay, D.M. (1971). Montague type semantics for modal logics with propositional quantifiers. Mathematical Logic Quarterly, 17(1), 245-249.

19. Fine, K. (1970). Propositional quantifiers in modal logic. Theoria, 36(3), 336-346.

20. Grover, D.L. (1972). Propositional quantifiers. Journal of Philosophical Logic, 1(2), 111-136.

21. Kaminski, M., \& Tiomkin, M. (1996). The expressive power of second-order propositional modal logic. Notre Dame Journal of Formal Logic, 37(1), 35-43.

22. Kuusisto, A. (2008). A modal perspective on monadic second-order alternation hierarchies. Advances in Modal Logic, 2008, 231-247.

23. Kuusisto, A. (2015). Second-order propositional modal logic and monadic alternation hierarchies. Annals of Pure and Applied Logic, 166(1), 1-28.

24. Ten Cate, B. (2006). Expressivity of second order propositional modal logic. Journal of Philosophical Logic, 35(2), 209-223.

25. Antonelli, G.A., \& Thomason, R.H. (2002). Representability in second-order propositional polymodal logic. The Journal of Symbolic Logic, 67(03), 1039-1054.

26. Kuhn, S. (2004). A simple embedding of T into double S5. Notre Dame Journal of Formal Logic, 45(1), 13-18.

27. Kremer, P. (1997). On the complexity of propositional quantification in intuitionistic logic. The Journal of Symbolic Logic, 62(02), 529-544.

28. Fritz, P. (2017). Logics for propositional contingentism. The Review of Symbolic Logic, 10(2), 203236.

29. Fritz, P. (2018). Propositional quantification in bimodal S5. Erkenntnis: An International Journal of Scientific Philosophy.

30. Kremer, P. (1993). Quantifying over propositions in relevance logic: nonaxiomatisability of primary interpretations of $\forall \mathrm{p}$ and $\exists$ p. The Journal of Symbolic Logic, 58(01), 334-349.

31. Kremer, P. (2018). Completeness of second-order propositional S4 and $\mathrm{H}$ in topological semantics. The Review of Symbolic Logic, 11(3), 507-518.

32. Ghilardi, S., \& Zawadowski, M. (1995). Undefinability of propositional quantifiers in the modal system S4. Studia Logica, 55(2), 259-271.

33. Zach, R. (2004). Decidability of quantified propositional intuitionistic logic and S4 on trees of height and arity $\leq \omega$. Journal of Philosophical Logic, 33(2), 155-164.

34. Belardinelli, F., \& Van Der Hoek, W. (2015). Epistemic quantified boolean logic: Expressiveness and completeness results. In 24th International Joint Conferences on Artificial Intelligence (IJCAI 2015) (pp. 2748-2754).

35. Belardinelli, F., van Ditmarsch, H., van der Hoek, W. (2016). Second-order propositional announcement logic. In Proceedings of the 2016 International Conference on Autonomous Agents \& Multiagent Systems (pp. 635-643): International Foundation for Autonomous Agents and Multiagent Systems.

36. Belardinelli, F., \& Van Der Hoek, W. (2016). A semantical analysis of second-order propositional modal logic. In Thirtieth AAAI Conference on Artificial Intelligence. 
37. Fan, T.-F., \& Liau, C.-J. (2017). Doxastic reasoning with multi-source justifications based on second order propositional modal logic. In Proceedings of the 16th Conference on Autonomous Agents and MultiAgent Systems (pp. 1529-1531): International Foundation for Autonomous Agents and Multiagent Systems.

38. Bednarczyk, B., \& Demri, S. (2019). Why propositional quantification makes modal logics on trees robustly hard? In 2019 34th Annual ACM/IEEE Symposium on Logic in Computer Science (LICS) (pp. 1-13): IEEE.

39. French, T. (2003). Quantified propositional temporal logic with repeating states. In 10th International Symposium on Temporal Representation and Reasoning, 2003 and Fourth International Conference on Temporal Logic. Proceedings (pp. 155-165): IEEE.

40. Holliday, W.H. (2017). A note on algebraic semantics for S5 with propositional quantifiers. Notre Dame Journal of Formal Logic.

41. Holliday, W.H., \& Litak, T. (2018). One modal logic to rule them all?. In Advances in Modal Logic 12, proceedings of the 12th conference on "Advances in Modal Logic," held in Bern, Switzerland, August 27-31, 2018 (pp. 367-386). http://www.aiml.net/volumes/volume12/Holliday-Litak.pdf.

42. Ding, Y. (2018). On the logics with propositional quantifiers extending S5П In Advances in Modal Logic 12, proceedings of the 12th conference on "Advances in Modal Logic," held in Bern, Switzerland, August 27-31, 2018 (pp. 219-235). http://www.aiml.net/volumes/volume12/Ding.pdf.

43. Davey, B.A., \& Priestley, H.A. (2002). Introduction to lattices and order. Cambridge university press.

44. Bezhanishvili, N. (2002). Pseudomonadic algebras as algebraic models of doxastic modal logic. Mathematical Logic Quarterly, 48(4), 624.

45. Fritz, P. (2016). Propositional contingentism. The Review of Symbolic Logic, 9(01), 123-142.

46. Gehrke, M., Harding, J., Venema, Y. (2006). Macneille completions and canonical extensions. Transactions of the American Mathematical Society, 358(2), 573-590.

47. Harding, J., \& Bezhanishvili, G. (2007). Macneille completions of modal algebras. Houston Journal of Mathematics, 33(2), 355-384.

48. Koppelberg, S. (1988). Algebraic theory. In Mond, J.D., \& Bonnet, R. (Eds.) Handbook of Boolean Algebras, Vol. 1: Elsevier.

49. Louveau, A. (1973). Caractérisation des sous-espaces compacts de $\beta n$. Bulletin des Sciences Mathematiques, 97.

50. Dow, A., \& Vermeer, J. (1992). Not all $\sigma$-complete boolean algebras are quotients of complete boolean algebras. Proceedings of the American Mathematical Society, 116(4), 1175-1177.

51. Vermeer, J. (1996). Quotients of complete boolean algebras. Annals of the New York Academy of Sciences, 788(1), 209-213.

52. Chang, C.C., \& Keisler, H.J. (1990). Model theory Vol. 73: Elsevier.

53. Givant, S., \& Halmos, P. (2008). Introduction to boolean algebras. Springer Science \& Business Media.

Publisher's Note Springer Nature remains neutral with regard to jurisdictional claims in published maps and institutional affiliations. 